\title{
Au-Catalyzed Formal Allylation of Diazo(thio)oxindoles: Application to Tandem Asymmetric Synthesis of Quaternary Stereocenters
}

\author{
Xiao-Yuan Cui, ${ }^{\dagger}$ Yu-Lei Zhao, ${ }^{\dagger}$ Yan-Mei Chen, ${ }^{\dagger}$ Su-Zhen Dong* $*{ }^{\dagger}$ Feng Zhou,${ }^{\dagger}$ Hai-Hong Wu*,${ }^{\dagger}$ \\ and Jian $\mathrm{Zhou}^{*, \dagger, \S}$
}

'Shanghai Engineering Research Center of Molecular Therapeutics and New Drug Development, and Shanghai Key Laboratory of Green Chemistry and Chemical Process, East China Normal University, Shanghai 200062, China.

${ }^{\ddagger}$ School of Chemistry and Chemical Engineering, Qufu Normal University, Qufu, 273165, China.

$\S$ State Key Laboratory of Organometallic Chemistry, Shanghai Institute of Organic Chemistry, Shanghai 200032, China.

Supporting Information

(Part I)

\begin{tabular}{|l|l|}
\hline Table of Contents & Page \\
\hline 1. General information & S2 \\
\hline 2. General procedure for the synthesis of diazothiooxindoles 1 & S3-S4 \\
\hline 3. Condition optimization of formal allylation of diazo(thio)oxindoles & S5-S10 \\
\hline 4. Condition optimization of asymmetric Michael addition & S11-S12 \\
\hline 5. General procedure for cationic Au(I) catalyzed formal allylation. & S13-S16 \\
\hline 6. General procedure for tandem formal allylation/Michael addition sequence & S17-S36 \\
\hline 7. Product elaboration & S37-S39 \\
\hline 8. X-ray crystallographic data of $\mathbf{1 1 r}$ and $\mathbf{1 3}$ & S40-S60 \\
\hline 9. Reference & S61 \\
\hline
\end{tabular}




\section{General information}

Reactions were monitored by thin layer chromatography using UV light to visualize the course of reaction. Infrared (IR) spectra were obtained using SHIMADZU TRT racer-100. The $[\alpha]_{\mathrm{D}}$ was recorded using Anton Paar MCP 5500. Chiral HPLC analysis was performed on a Shimadzu LC-20AD instrument using Daicel chiral columns at room temperature and a mixture of HPLC-grade hexane and isopropanol as eluent. ${ }^{1} \mathrm{H},{ }^{13} \mathrm{C}$ and ${ }^{19} \mathrm{~F}$ spectra were obtained using a Bruker DPX-400 or Bruker DPX-500 spectrometer. The Bruker Maxis impact is used for HRMS measurement with TOF analysis. Chemical shifts were reported in ppm from $\mathrm{CDCl}_{3}$ or $\mathrm{CD}_{2} \mathrm{Cl}_{2}$ with the TMS as the internal standard. The following abbreviations were used to designate chemical shift multiplicities: $\mathrm{s}=$ singlet, $\mathrm{d}=$ doublet, $\mathrm{t}=$ triplet, $\mathrm{q}=$ quartet, $\mathrm{h}=$ heptet, $\mathrm{m}=$ multiplet, $\mathrm{br}=$ broad .

All reactions were performed under an atmosphere of $\mathrm{N}_{2}$ and oil bath was used as heat source as needed. Anhydrous $\mathrm{CH}_{2} \mathrm{Cl}_{2}$ was prepared by first distillation over $\mathrm{P}_{2} \mathrm{O}_{5}$ and then from $\mathrm{CaH}_{2}$. Anhydrous $\mathrm{Et}_{2} \mathrm{O}$ and toluene were prepared by distillation over sodium-benzophenone ketyl prior to use. $\mathrm{Ph}_{3} \mathrm{PAuCl}$ and ${ }^{t} \mathrm{Bu}_{2} \mathrm{CyPAuCl},{ }^{1} \mathbf{C 1 - C 6},{ }^{2}$ diazo(thio)oxindoles, ${ }^{3}$ as well as nitroenynes ${ }^{4}$ were synthesized according to literature procedures. AgOTf was purchased from TCI company and used without any purification. Allyltrimethylsilane was purchased from Shaoyuan Chemical (Shanghai) Co. Ltd and purified by distillation and then stored in $5 \AA ̊$ molecular sieves.

\section{List of abbreviation:}

\begin{tabular}{ccc}
\hline Entry & Chemical name & Abbreviation \\
\hline 1 & Petroleum ether & PE \\
2 & Ethyl acetate & EtOAc \\
3 & $N, N$-Dimethylacetamide & $\mathrm{DMA}$ \\
4 & Dichloromethane & $\mathrm{CH}_{2} \mathrm{Cl}_{2}$ \\
5 & Tetrahydrofuran & $\mathrm{THF}$ \\
\hline
\end{tabular}




\section{General procedure for the synthesis of diazothiooxindoles 1 .}
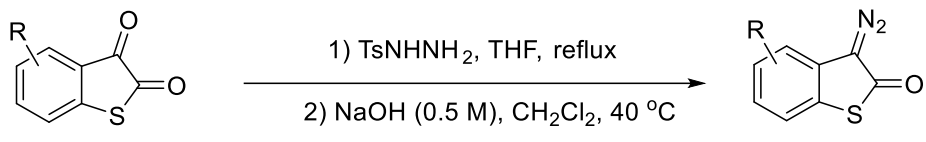

1

Under an atmosphere of $\mathrm{N}_{2}$, to a flame-dried $25 \mathrm{~mL}$ three-necked flask were added thiooxindoles $(1.0 \mathrm{mmol}), \mathrm{TsNHNH}_{2}(186.2 \mathrm{mg}, 1.0 \mathrm{mmol})$ and $3 \mathrm{~mL}$ THF. The mixture was refluxed for $2 \mathrm{~h}$, then cooled to room temperature. The reaction mixture was concentrated under reduced pressure, and then redissolved in $10 \mathrm{~mL} \mathrm{CH}_{2} \mathrm{Cl}_{2}$. After addition of aq. $\mathrm{NaOH}(0.5 \mathrm{M}, 10 \mathrm{~mL})$, the resulting mixture was stirred at $40^{\circ} \mathrm{C}$ for $2 \mathrm{~h}$. Water $(30 \mathrm{~mL})$ and $\mathrm{CH}_{2} \mathrm{Cl}_{2}(30 \mathrm{~mL})$ were added and the organic layer was separated. The aqueous layer was extracted with $\mathrm{CH}_{2} \mathrm{Cl}_{2}(5 \times 10 \mathrm{~mL})$, then the organic phase was combined, washed with brine $(10 \mathrm{~mL})$, dried over anhydrous $\mathrm{Na}_{2} \mathrm{SO}_{4}$ and concentrated to give the crude residue, which was purified by column chromatography eluting with EtOAc/PE (1:10, v/v) to give the desired diazothiooxindoles $\mathbf{1}$.

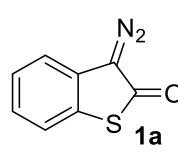

1a was obtained in $158.4 \mathrm{mg}$ (90\% yield) as reddish-brown solid. Mp $62-64{ }^{\circ} \mathrm{C} .{ }^{1} \mathrm{H}$ NMR (400 MHz, $\left.\mathrm{CDCl}_{3}\right): \delta$ 7.40-7.37 (m, 1H), 7.32-7.28 (m, 1H), 7.24-7.20 (m, 1H), 7.16-7.14 (m, 1H); ${ }^{13} \mathrm{C}$ NMR (100 MHz, $\left.\mathrm{CDCl}_{3}\right): \delta 187.4,127.0,126.4,126.1,124.8$, 123.6, 118.3; IR (Neat): 2131, 1641, 1626, 1466, 1368, 1304, 1209, 887, $750 \mathrm{~cm}^{-1}$; HRMS (ESI): Exact mass calcd for $\mathrm{C}_{8} \mathrm{H}_{4} \mathrm{~N}_{2} \mathrm{NaOS}[\mathrm{M}+\mathrm{Na}]^{+}:$198.9937, Found: 198.9932 .

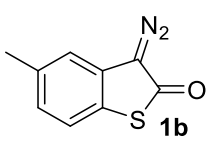

1b was obtained in $167.2 \mathrm{mg}$ (88\% yield) as reddish-brown solid. $\mathrm{Mp} 85-86{ }^{\circ} \mathrm{C} .{ }^{1} \mathrm{H}$ NMR (400 MHz, $\left.\mathrm{CDCl}_{3}\right): \delta 7.28-7.26(\mathrm{~m}, 1 \mathrm{H}), 7.04(\mathrm{dd}, J=7.8,1.6 \mathrm{~Hz}, 1 \mathrm{H}), 6.97$ (s, $1 \mathrm{H}), 2.39(\mathrm{~s}, 3 \mathrm{H}) ;{ }^{13} \mathrm{C} \mathrm{NMR}\left(100 \mathrm{MHz}, \mathrm{CDCl}_{3}\right): \delta 187.9,136.5,127.2,124.7,123.6$, 123.3, 118.9, 21.4; IR (Neat): 2099, 1657, 1460, 1348, 1290, 1211, 1186, 812, $804 \mathrm{~cm}^{-1}$; HRMS (ESI): Exact mass calcd for $\mathrm{C}_{9} \mathrm{H}_{6} \mathrm{~N}_{2} \mathrm{NaOS}[\mathrm{M}+\mathrm{Na}]^{+}:$213.0093, Found: 213.0090 .

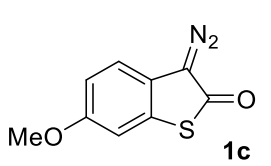

1c was obtained in $119.5 \mathrm{mg}$ (58\% yield) as reddish-brown solid. Mp $93-95{ }^{\circ} \mathrm{C} .{ }^{1} \mathrm{H}$ NMR (400 MHz, CDCl $): \delta 7.06(\mathrm{~d}, J=8.6 \mathrm{~Hz}, 1 \mathrm{H}), 6.98(\mathrm{~d}, J=2.4 \mathrm{~Hz}, 1 \mathrm{H}), 6.88$ (dd, $J=8.6 \mathrm{~Hz}, 2.4 \mathrm{~Hz}, 1 \mathrm{H}), 3.82(\mathrm{~s}, 3 \mathrm{H}) ;{ }^{13} \mathrm{C} \mathrm{NMR}\left(100 \mathrm{MHz}, \mathrm{CDCl}_{3}\right): \delta 188.1$, 158.5, 128.0, 119.2, 116.3, 113.4, 109.3, 55.7; IR (Neat): 2095, 1666, 1646, 1487, 1251, 1068, 1044, 862, $809 \mathrm{~cm}^{-1}$; HRMS (ESI): Exact mass calcd for $\mathrm{C}_{9} \mathrm{H}_{6} \mathrm{~N}_{2} \mathrm{NaO}_{2} \mathrm{~S}[\mathrm{M}+\mathrm{Na}]^{+}:$229.0042, Found: 229.0035 . 


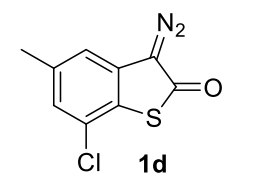

1d was obtained in $152.3 \mathrm{mg}\left(68 \%\right.$ yield) as reddish-brown solid. Mp $128-130{ }^{\circ} \mathrm{C} .{ }^{1} \mathrm{H}$ NMR (400 MHz, $\left.\mathrm{CDCl}_{3}\right): \delta$ 7.06-7.05 (m, 1H), 6.89-6.87 (m, 1H), $2.38(\mathrm{~s}, 3 \mathrm{H}) ;{ }^{13} \mathrm{C}$ NMR (100 MHz, $\left.\mathrm{CDCl}_{3}\right): \delta 186.3,138.0,128.6,126.7,126.1,122.9,117.0,21.2$; IR (Neat): 2123, 1660, 1631, 1557, 1377, 1230, 1217, 1051, 876, $837 \mathrm{~cm}^{-1}$; HRMS (ESI): Exact mass calcd for $\mathrm{C}_{9} \mathrm{H}_{5} \mathrm{ClN}_{2} \mathrm{NaOS}[\mathrm{M}+\mathrm{Na}]^{+}:$246.9703, Found: 246.9697.

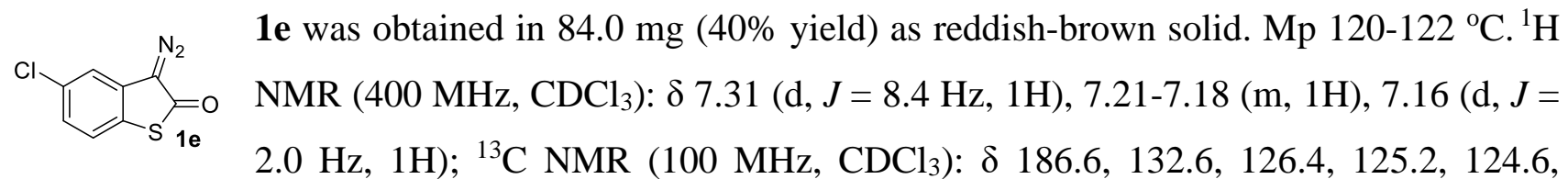
118.3; IR (Neat): 2135, 1672, 1630, 1458, 1335, 1204, 1090, 797, $772 \mathrm{~cm}^{-1}$; GC-MS (EI): 210 (M+, 48), 184 (14), 182 (33), 156 (33), 154 (85), 119 (100), 75 (12), 69 (25); HRMS (TOF, EI): Exact mass calcd for $\mathrm{C}_{8} \mathrm{H}_{3} \mathrm{ClN}_{2} \mathrm{OS}: 209.9655$, Found: 209.9658 . 


\section{Condition optimization of formal allylation of diazo(thio)oxindoles.}

(a) Condition optimization of formal allylation of diazothiooxindoles

First of all, the performance of Ru complex was studied, but only cyclopropanation product $\mathbf{9}$ could be detected. With the addition of $1.0 \mathrm{~mol} \% \mathrm{TfOH}$ to facilitate the ring opening process, $6 \mathrm{a}$ could be obtained in $15 \%$ and $60 \%$ yield respectively, with $\mathrm{Rh}_{2}(\mathrm{OAc})_{4}$ and $\mathrm{Rh}_{2}(\mathrm{TFA})_{4}$ as catalyst (entries 1-2, Table S1). The influence of other metal salts were also investigated, but no reaction occurred with $\mathrm{AgOTf}, \mathrm{Hg}(\mathrm{OTf})_{2}$ or $\mathrm{Pd}(\mathrm{OTf})_{2}$ as catalyst, and $10 \mathrm{~mol} \% \mathrm{Cu}(\mathrm{OTf})_{2}$ gave only trace amounts of $\mathbf{6 a}$ (entries 3-6). ${ }^{5}$

Based on our efforts in $\mathrm{Au}(\mathrm{I})$ catalyzed transformation of diazooxindoles, ${ }^{6}$ a series of cationic $\mathrm{Au}$ salts for the formal allylation of diazothiooxindole $\mathbf{1 a}$ with allyltrimethylsilane $\mathbf{2 a}$ were investigated. The IPrAuOTf proved to be workable to deliver the desired 3-allyl thiooxindole $6 \mathbf{a}$ in $52 \%$ isolated yield after $10 \mathrm{~h}$, without the detection of cyclopropane intermediate 9 (entry 7).

The influence of counter anion was also investigated, but no reaction occurred with $\mathrm{IPrAuBF}_{4}$, $\mathrm{IPrAuPF}_{6}$ or $\mathrm{IPrAuNTf}_{2}$ as catalyst (entries 8-10). When performing the reaction under room temperature, only trace amount of $6 \mathbf{a}$ could be obtained with IPrAuOTf as catalyst (entry 11). Then the gold complex bearing different ligands were analyzed. It turned out that phosphine ligands played an important role. While no reaction occurred when $\mathrm{Ph}_{3} \mathrm{PAuOTf}$ was used, the inclusion of (JohnPhos)AuOTf gave 3-allyl thiooxindole 6a in 49\% yield after $6 \mathrm{~h}$ (entries 11-13). To our delight, in situ generated ${ }^{t} \mathrm{Bu}_{2} \mathrm{CyPAuOTf}$ could catalyze the reaction efficiently at $25{ }^{\circ} \mathrm{C}$ to give $6 \mathbf{a}$ in $80 \%$ within 11 hours (entry 15). Consequently, the in situ generated ${ }^{t} \mathrm{Bu}_{2} \mathrm{CyPAuOTf}$ was employed to be the optimal catalyst for the formal allylation of diazothiooxindoles, which could deliver $\mathbf{6 a}$ in $76 \%$ yield within 18 hours, even in the presence of 5.0 equiv of allyltrimethylsilane $\mathbf{2 a}$ with 2 mol\% catalyst loading (entry 16). Lowering the temperature and changing the solvent can not improve the result (entries 17-18).

The cyclopropane intermediate 9 was isolated as yellow oil. ${ }^{1} \mathrm{H}$ NMR (400 $\left.\mathrm{MHz}, \mathrm{CDCl}_{3}\right)$ : $\delta$ 7.46-7.43 (m, 1H), 7.29-7.23 (m, 3H), 6.93-6.91 (m, 1H), 2.25 (ABd, $J=9.2,4.4$ Hz, 1H), 2.08-2.03 $(\mathrm{m}, 1 \mathrm{H}), 1.46(\mathrm{ABd}, J=8.4,4.4 \mathrm{~Hz}, 1 \mathrm{H}), 0.84-0.82(\mathrm{~m}, 2 \mathrm{H}), 0.00(\mathrm{~s}, 9 \mathrm{H}) ;{ }^{13} \mathrm{C} \mathrm{NMR}(100 \mathrm{MHz}$, $\mathrm{CDCl}_{3}$ ): $\delta 205.1,137.6,137.3,128.5,126.9,124.8,123.6,44.9,40.2,30.7,16.7,0.0$; IR (ATR): 2361, 2342, 1696, 1468, 1368, 1250, 1092, $1049 \mathrm{~cm}^{-1}$; HRMS (ESI): Exact mass calcd for $\mathrm{C}_{14} \mathrm{H}_{18} \mathrm{NaOSSi}$ $[\mathrm{M}+\mathrm{Na}]^{+}:$285.0740, Found: 285.0733. 
Table S1. Condition optimization of formal allylation of diazothiooxindoles.

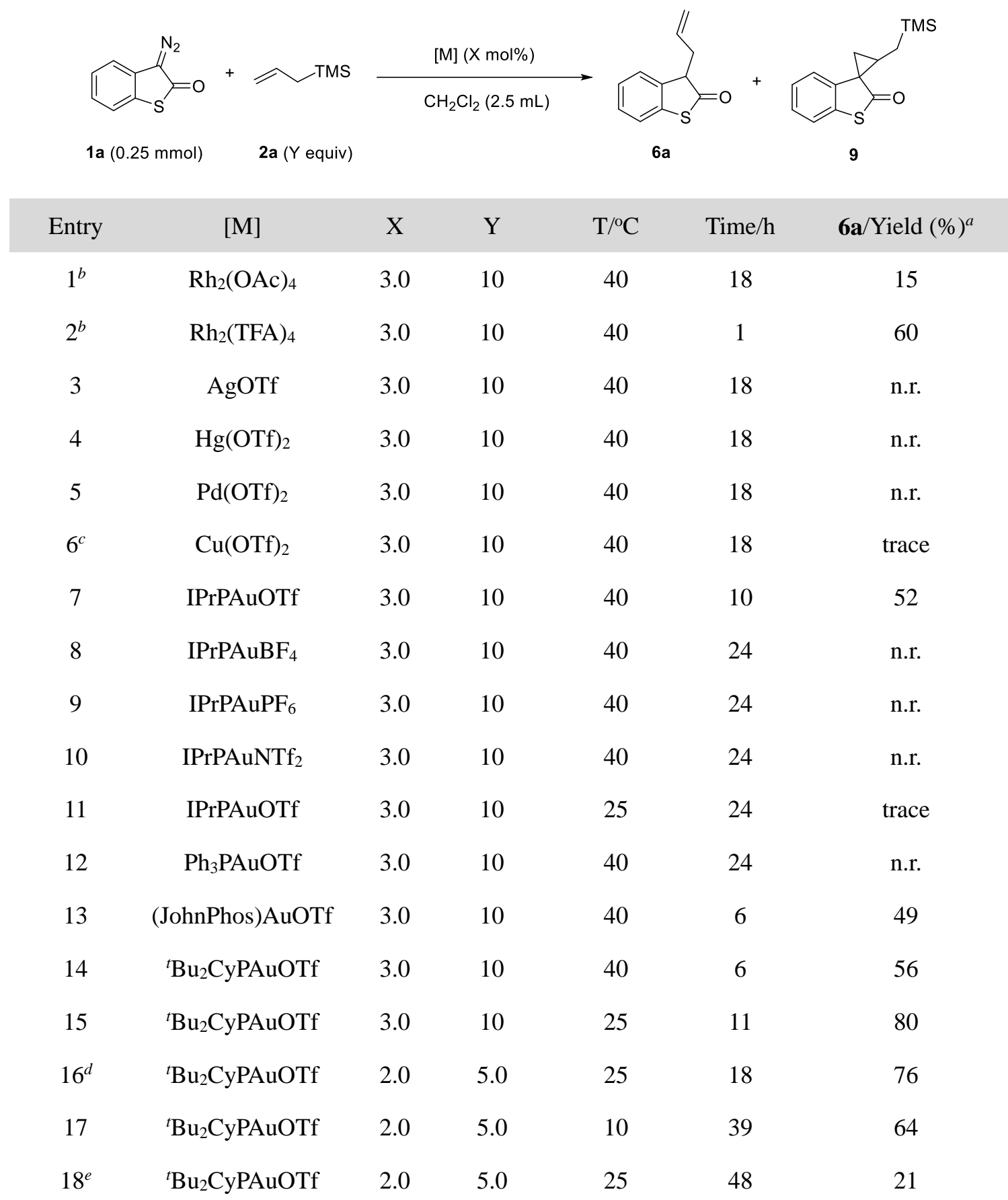

${ }^{a}$ Isolated yield. ${ }^{b} \mathbf{1 a}(0.25 \mathrm{mmol})$, with $\mathrm{CH}_{2} \mathrm{Cl}_{2}(3.0 \mathrm{~mL}), 5.0$ equiv of the allyltrimethylsilane, then addition of $1 \mathrm{~mol} \% \mathrm{TfOH}$ at rt. ${ }^{c} \mathrm{Cu}(\mathrm{OTf})_{2}(10 \mathrm{~mol} \%) .{ }^{d} \mathbf{1 a}(0.25 \mathrm{mmol})$, with $\mathrm{CH}_{2} \mathrm{Cl}_{2}(2.5 \mathrm{~mL}), 5.0$ equiv of the allyltrimethylsilane and ${ }^{t} \mathrm{Bu}_{2} \mathrm{CyPAuOTf}$ prepared by anion exchange of ${ }^{t} \mathrm{Bu}_{2} \mathrm{CyPAuCl}(2.0 \mathrm{~mol} \%)$ with $\operatorname{AgOTf}(3.0 \mathrm{~mol} \%) .{ }^{e} 1,2$-dichloroethane as solvent. 


\section{(b) Optimization condition of formal allylation of diazooxindoles}

For the formation allylation of diazooxindoles, we also compared $\mathrm{Rh}_{2}(\mathrm{OAc})_{4}$ and $\mathrm{Ph}_{3} \mathrm{PAuOTf}$ in the reaction of diazooxindole $\mathbf{8 c}(0.2 \mathrm{mmol})$ using allyltrimethylsilane $\mathbf{2 a}(2.0 \mathrm{mmol})$, which was carried out in $4.0 \mathrm{~mL}$ of anhydrous $\mathrm{CH}_{2} \mathrm{Cl}_{2}$, in the presence of $1.0 \mathrm{~mol} \%$ of the specific metal salt. It was found that at $0{ }^{\circ} \mathrm{C}, \mathrm{Rh}_{2}(\mathrm{OAc})_{4}$ failed to catalyze the cyclopropanation even after $24 \mathrm{~h}$ (entry 1 , Table S2), but it could catalyze this reaction slowly at $25{ }^{\circ} \mathrm{C}$, as it took about 48 hours for the full consumption of diazooxindole 8c by TLC analysis, affording the cyclopropane intermediate $\mathbf{1 4}$ in 44\% NMR yield (entry 2). After the completion of cyclopropanation, the solvent and excess allyltrimethylsilane $\mathbf{2 a}$ was removed under reduced pressure, as the presence of allyltrimethylsilane $\mathbf{2 a}$ slowed down the ring-opening step, possibly due to its interaction with the metal salt. ${ }^{7}$ Then $4.0 \mathrm{~mL}$ of ordinary $\mathrm{CH}_{2} \mathrm{Cl}_{2}$ (not anhydrous) was added, and the mixture was stirred at $25{ }^{\circ} \mathrm{C}$ for $24 \mathrm{~h}$, and no 3-allyloxindole 7c was observed by NMR analysis.

Table S2. Comparing $\mathrm{Rh}_{2}(\mathrm{OAc})_{4}$ and $\mathrm{Ph}_{3} \mathrm{PAuOTf}$ for the reaction of $\mathbf{8 c}$ and $\mathbf{2 a}$

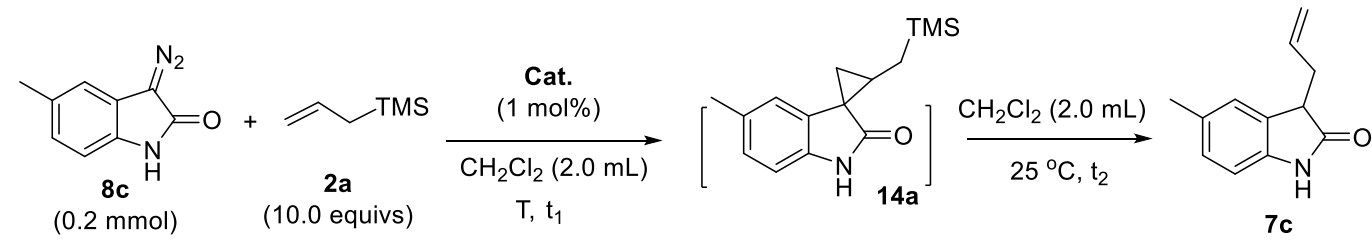

\begin{tabular}{|c|c|c|c|c|c|c|}
\hline Entry & Cat. & $\mathrm{T}\left({ }^{\circ} \mathrm{C}\right)$ & $\mathrm{t}_{1}(\mathrm{~h})$ & Yield of 14a (\%) & $\mathrm{t}_{2}(\mathrm{~h})$ & Yield of $7 \mathbf{c}(\%)$ \\
\hline 1 & $\mathrm{Rh}_{2}(\mathrm{OAc})_{4}$ & 0 & 24 & NR & - & - \\
\hline 2 & $\mathrm{Rh}_{2}(\mathrm{OAc})_{4}$ & 25 & 48 & 44 (NMR) & 24 & NR \\
\hline 3 & $\mathrm{Ph}_{3} \mathrm{PAuOTf}$ & 0 & 24 & 75 (NMR) & 24 & 68 \\
\hline 4 & $\mathrm{Ph}_{3} \mathrm{PAuOTf}$ & 25 & 3 & 41 (NMR) & 24 & 32 (NMR) \\
\hline 5 & ${ }^{t} \mathrm{Bu}_{2} \mathrm{CyPAuOTf}$ & 0 & 24 & 53 (NMR) & 31 & 30 (NMR) \\
\hline
\end{tabular}

It turned out that the use of $1.0 \mathrm{~mol} \% \mathrm{Ph}_{3} \mathrm{PAuOTf}$, prepared in situ from $1.0 \mathrm{~mol} \% \mathrm{Ph}_{3} \mathrm{PAuCl}$ and $1.5 \mathrm{~mol} \%$ AgOTf, catalyzed the initial cyclopropanation more efficiently, which could be carried out at $0{ }^{\circ} \mathrm{C}$ to provide the cyclopropane 14a in $75 \%$ NMR yield. The following ring-opening step worked well at $25{ }^{\circ} \mathrm{C}$, by the same manipulation described above, to give the desired 3-allyloxindole 7c in $68 \%$ isolated yield (entry 3). However, when run at $25^{\circ} \mathrm{C}$, the initial cyclopropantion could finish within $3 \mathrm{~h}$ to give $\mathbf{1 4 a}$ in diminished $41 \%$ NMR yield, due to the presence of side reactions (entry 4). 
${ }^{t} \mathrm{Bu}_{2} \mathrm{CyPAuOTf}$ gave the desired 3-allyloxindole $7 \mathbf{c}$ in 30\% NMR yield (entry 5).

The cyclopropane intermediate 14a was obtained as white powder (m.p. 134-136 ${ }^{\circ} \mathrm{C}$ ). ${ }^{1} \mathrm{H}$ NMR (400 MHz, $\left.\mathrm{CDCl}_{3}\right): \delta 9.35(\mathrm{~s}, 1 \mathrm{H}), 6.99-6.97(\mathrm{~m}, 1 \mathrm{H}), 6.91(\mathrm{~s}, 1 \mathrm{H}), 6.87-6.85(\mathrm{~m}, 1 \mathrm{H}), 2.29(\mathrm{~s}, 3 \mathrm{H})$, 1.79-1.73 (m, 2H), 1.31-1.27 (m, 1H), 1.01-0.95 (m, 1H), 0.89-0.84 (m, 1H), -0.01 (s, 9H); ${ }^{13} \mathrm{C} \mathrm{NMR}$ (100 MHz, $\left.\mathrm{CDCl}_{3}\right): \delta$ 178.8, 141.0, 130.7, 129.9, 127.5, 122.9, 109.9, 33.2, 25.4, 21.3, 21.3, 15.7, -1.6; IR (ATR): 3031, 2956, 1703, 1630, 1486, 1372, 1250, $1192 \mathrm{~cm}^{-1}$; HRMS (ESI): Exact mass calcd for $\mathrm{C}_{15} \mathrm{H}_{22} \mathrm{NOSi}[\mathrm{M}+\mathrm{H}]^{+}: 260.1465$, Found: 260.1467 .

It was found that the electron-withdrawing substituents such as fluoro and chloro group on the framework of diazooxindole $\mathbf{8}$ could accelerate both the cyclopropanation and ring-opening step, which allowed the usage of allyltrimethylsilane to be reduced to 5.0 equiv. For example, in the case of 5-fluorodiazooxindole 8a, the cyclopropanation step could finish within $2 \mathrm{~h}$ at $0{ }^{\circ} \mathrm{C}$, and the ring-opening completed within $1 \mathrm{~h}$ to give 3-allyloxindole 7a in 79\% yield. In the case of 4,6-dichloro diazooxindole $8 \mathbf{d}$ that had a poor solubility in $\mathrm{CH}_{2} \mathrm{Cl}_{2}$, the cyclopropanation finished within $15 \mathrm{~h}$ at 0 ${ }^{\circ} \mathrm{C}$, in spite of the unfavored steric hindrance resulting from the $\mathrm{C} 4$ chloro substitution, and the ring-opening could finish within $0.5 \mathrm{~h}$ to afford 3 -allyloxindole $\mathbf{7 d}$ in $88 \%$ yield.
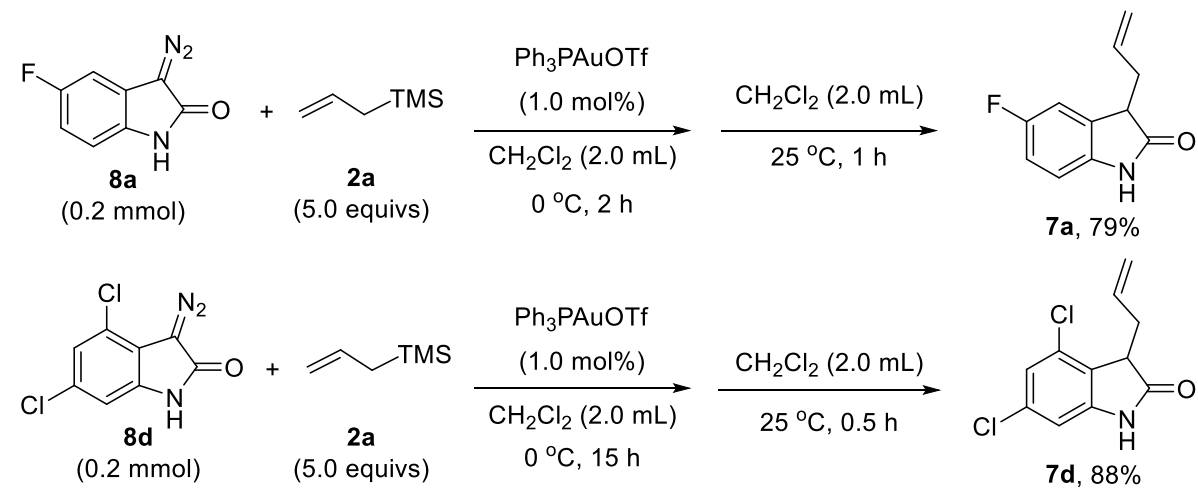

We also investigated the role of the $\mathrm{Ph}_{3} \mathrm{PAuOTf}$ in the ring-opening reaction of cyclopropane 14 by using cyclopropane 14b that could be obtained in high yield. It was observed that when anhydrous $\mathrm{CH}_{2} \mathrm{Cl}_{2}$ was used as the solvent, no ring-opening of $\mathbf{1 4 \mathbf { b }}$ was detected by TLC analysis, no matter using $\mathrm{Rh}_{2}(\mathrm{OAc})_{4}$, AgOTf or $\mathrm{Ph}_{3} \mathrm{PAuOTf}$ as the catalyst (entries 1-3, Table S3). On the other hand, when the reaction was carried out in anhydrous $\mathrm{CH}_{2} \mathrm{Cl}_{2}$, with the addition of 1.0 equiv of water, $\mathrm{AgOTf}$ and $\mathrm{Rh}_{2}(\mathrm{OAc})_{4}$ still failed to mediate the ring-opening (entries 4-5), but $\mathrm{Ph}_{3} \mathrm{PAuOTf}$ could promote the reaction in 27\% conversion in 6 hours (entry 6). In addition, when using ordinary commercial $\mathrm{CH}_{2} \mathrm{Cl}_{2}$ (not anhydrous) as the solvent, the ring-opening of $\mathbf{1 4 b}$ catalyzed by $2.3 \mathrm{~mol} \%$ of HOTf proceeded smoothly, with full conversion achieved within 4 hours to afford $7 \mathbf{a}$ in $80 \%$ yield (entry 7). However, it took about 11 hours for $\mathrm{Ph}_{3} \mathrm{PAuOTf}$ (prepared form $1.0 \mathrm{~mol} \%$ of $\mathrm{Ph}_{3} \mathrm{PAuCl}$ and 
$1.5 \mathrm{~mol} \%$ of AgOTf) to achieve $68 \%$ conversion by ${ }^{1} \mathrm{H}$ NMR analysis (entry 8). Even using wet $\mathrm{CH}_{2} \mathrm{Cl}_{2}$ as the solvent, $\mathrm{Rh}_{2}(\mathrm{OAc})_{4}$ and AgOTf still failed to mediate the ring-opening (entries 9-10). These results suggested that water might play a role in the ring-opening reaction. However, whether the ring opening of cyclopropane $\mathbf{1 4}$ was mediated by $\mathrm{Au}(\mathrm{I})$ species or by HOTf generated in situ from the hydrolysis of $\mathrm{Ph}_{3} \mathrm{PAuOTf}$ waited for further studies.

Table S3. Investigate the role of $\mathrm{Au}(\mathrm{I})$ catalyst in the ring-opening of cyclopropane 14b

\begin{tabular}{|c|c|c|c|c|c|}
\hline Entry & $\mathrm{CH}_{2} \mathrm{Cl}_{2}$ & Catalyst & $\begin{array}{l}\mathrm{H}_{2} \mathrm{O} \\
\text { (equiv) }\end{array}$ & Time (h) & Comment \\
\hline 1 & anhydrous & $\mathrm{Rh}_{2}(\mathrm{OAc})_{4}(1.0 \mathrm{~mol} \%)$ & 0 & 6 & No reaction \\
\hline 2 & anhydrous & $\mathrm{Ph}_{3} \mathrm{PAuOTf}(1.0 \mathrm{~mol} \%)$ & 0 & 6 & No reaction \\
\hline 3 & anhydrous & AgOTf (1.5 mol\%) & 0 & 6 & No reaction \\
\hline 4 & anhydrous & AgOTf (1.5 mol\%) & 1.0 & 6 & No reaction \\
\hline 5 & anhydrous & $\mathrm{Rh}_{2}(\mathrm{OAc})_{4}(1.0 \mathrm{~mol} \%)$ & 1.0 & 6 & No reaction \\
\hline 6 & anhydrous & $\mathrm{Ph}_{3} \mathrm{PAuOTf}(1.0 \mathrm{~mol} \%)$ & 1.0 & 6 & $27 \%$ conv. $^{a}$ \\
\hline 7 & ordinary & HOTf (2.3 mol\%) & 0 & 4 & $80 \%$ yield \\
\hline 8 & ordinary & $\mathrm{Ph}_{3} \mathrm{PAuOTf}(1.0 \mathrm{~mol} \%)$ & 0 & 11 & $68 \%$ conv. $^{a}$ \\
\hline 9 & ordinary & AgOTf (1.5 mol\%) & 0 & 6 & No reaction \\
\hline 10 & ordinary & $\mathrm{Rh}_{2}(\mathrm{OAc})_{4}(1.0 \mathrm{~mol} \%)$ & 0 & 6 & No reaction \\
\hline
\end{tabular}

${ }^{a}$ Determined by ${ }^{1} \mathrm{H}$ NMR analysis of the crude reaction mixture. 


\section{(c) Comparison with methods for allylic alkylation of (thio)oxindole under basic condition}

To further illustrate the superiority of the $\mathrm{Au}(\mathrm{I})$ catalyzed formal allylation, the synthesis of 3-allyl (thio)oxindole $6 \mathbf{a}$ or $7 \mathbf{a}$ via the allylation of (thio)oxindole 5a or $\mathbf{5 b}$ under typical basic conditions were performed. As shown in Table S4, the use of ${ }^{n} \mathrm{BuLi} / \mathrm{TMEDA}$ for the alkylation of thiooxindole 5a with 1.2 equiv of allyl bromide $\mathbf{2 b}$ gave $\mathbf{6 a}$ in only $19 \%$ yield, with 3,3-diallyl thiooxindole $\mathbf{6 a}$ ' as major product (entry 1). Changing ${ }^{n} \mathrm{BuLi}$ to LDA gave similar result (entry 2). The Pd-catalyzed allylation of thiooxindole 5a with 1.2 equiv of allyl acetate $\mathbf{2 c}$ was also tried, but only $7 \%$ yield of $\mathbf{6 a}$ could be obtained, with the formation of 3,3-diallyl thiooxindole $6 \mathbf{a}^{\prime}$ in $52 \%$ yield (entry 3 ).

The side diallylation was found to be severe in the case of oxindoles with electron-withdrawing substituents, as exemplified by the reaction of 5-fluorooxindole 5b (entry 4-6). Driven by electronic effect of aromatic ring, the deprotonation of 3-allyl oxindoles with an electron-withdrawing substituent might occur readily to give planar enolate intermediates for alkylation, as a result, it difficult to realize selective mono-allylation under basic conditions.

Table S4. The comparison with traditional allylation reaction

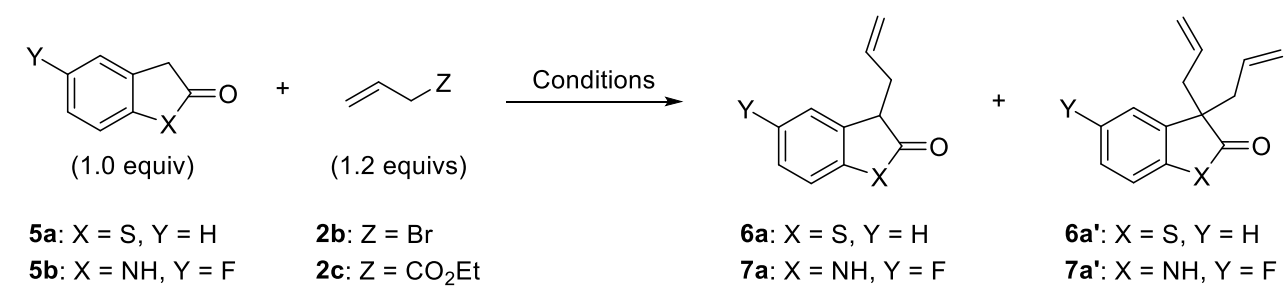

\begin{tabular}{|c|c|c|c|c|c|}
\hline Entry & Subtrate & Conditions & Conv. $/ \%$ & $\begin{array}{c}\text { 6a or 7a } \\
/ \text { Yield }(\%)^{a}\end{array}$ & $\begin{array}{l}\text { 6a' or 7a' } \\
/ \text { Yield }(\%)^{a}\end{array}$ \\
\hline 1 & $5 \mathbf{a}+2 \mathbf{b}$ & ${ }^{n} \mathrm{BuLi}$ (1.2 eq.), TMEDA/THF, -78 to $25^{\circ} \mathrm{C}$ & 90 & 19 & 46 \\
\hline 2 & $\mathbf{5 a}+\mathbf{2 b}$ & LDA (1.2 eq.)/THF, -78 to $25^{\circ} \mathrm{C}$ & 93 & 21 & 49 \\
\hline 3 & $5 \mathbf{a}+2 \mathbf{c}$ & $\mathrm{Pd}\left(\mathrm{PPh}_{3}\right)_{4}(5.0 \mathrm{~mol} \%), \mathrm{NaH} /$ Toluene, $25^{\circ} \mathrm{C}$ & 90 & 7 & 52 \\
\hline 4 & $5 b+2 b$ & ${ }^{n} \mathrm{BuLi}$ (1.2 eq.), TMEDA/THF, -78 to $25^{\circ} \mathrm{C}$ & 80 & 20 & 36 \\
\hline 5 & $5 \mathbf{b}+2 \mathbf{b}$ & LDA (1.2 eq.)/THF, -78 to $25^{\circ} \mathrm{C}$ & 98 & 15 & 52 \\
\hline 6 & $5 b+2 c$ & $\mathrm{Pd}\left(\mathrm{PPh}_{3}\right)_{4}(5.0 \mathrm{~mol} \%), \mathrm{NaH} /$ Toluene, $25^{\circ} \mathrm{C}$ & 76 & 10 & 55 \\
\hline
\end{tabular}




\section{Condition optimization of asymmetric Michael addition}

Previously, we found that simple cinchonidine derived bifunctional phosphoramide catalyst C2 was a powerful catalyst for the Michael addition of 3-substituted oxindoles to $\beta$-aryl or alkyl substituted nitrolefins. ${ }^{2}$ Based on these results, we first examined the performance of chiral phosphoramides C1-3 bearing different substituents for the enantioselective Michael addition of 3-allyl thiooxindole 6a with nitrodiene 10a. As shown in Table S5, chiral C1 could catalyzed the reaction smoothly in toluene at $-40{ }^{\circ} \mathrm{C}$ with MS $5 \AA$ as additive, to give the desired chiral $\mathbf{4 a}$ in $97 \%$ yield, 93/7 dr and 72\% ee (entry 1). Further variation of the diisopropyl substituted $\mathbf{C 1}$ to $\mathbf{C 2}$ and $\mathbf{C 3}$ bearing diethyl and diisopentyl moiety gave lower $60 \%$ and $43 \%$ ee, respectively (entries $2-3$ ). The performance of chiral bifunctional catalysts C4-C6 bearing other kind of H-bonding moiety, such as, squaric amide, urea and amide, were also investigated, but inferior results were obtained in terms of diastereoselectivity and enantioselectivity (entries 4-6).

Table S5. Catalyst evaluation for the asymmetric Michael addition

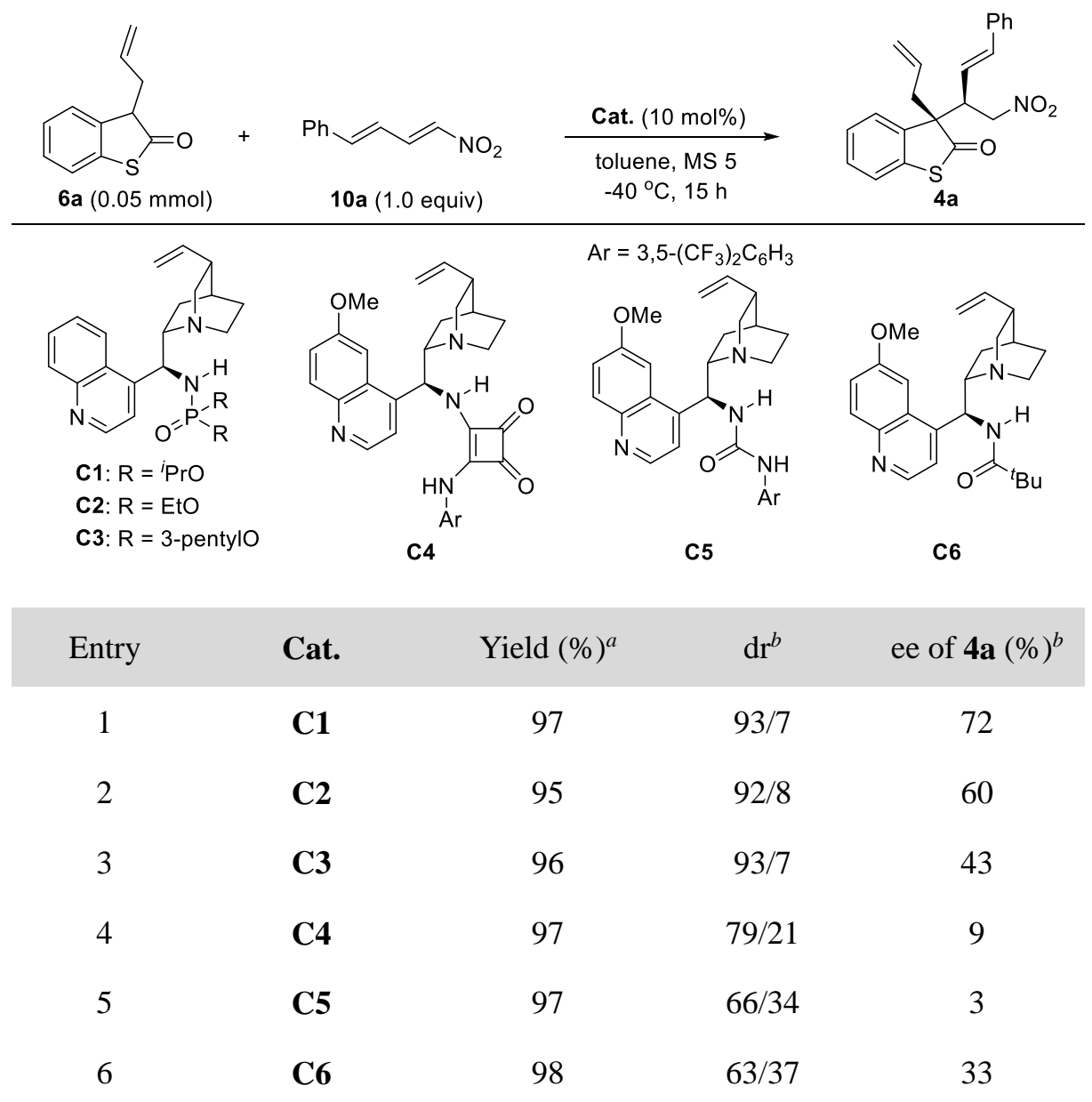

${ }^{a}$ Isolated yield. ${ }^{b}$ Ee and Dr were determined by chiral HPLC analysis. 
Using phosphoramide $\mathbf{C 1}$ as catalyst, the solvent effects were examined, with typical results shown in Table S6. By using THF, EtOAc, $\mathrm{CH}_{2} \mathrm{Cl}_{2}$, acetonitrile, acetone or $\mathrm{Et}_{2} \mathrm{O}$ as solvent, the desired product $4 \mathbf{a}$ was obtained in $29-70 \%$ enantioselectivity, which were much lower than that obtained in toluene (entries $2-7$ vs 1 ). Then, lowering the temperature to $-78^{\circ} \mathrm{C}$, the ee value could be improved to $90 \%$ (entry 8 ). Then we tried using additive to improve the stereoselectivities, and found that the powdered $3 \AA$ MS could gave $4 \mathbf{a}$ in $95 \%$ yield with $92 \%$ ee (entry 9). Taking into consideration of both reactivity and stereoselectivity, we determined to perform the Michael addition reaction in toluene at $-78{ }^{\circ} \mathrm{C}$ with the addition of $3 \AA \mathrm{MS}$, in the presence of $10 \mathrm{~mol} \%$ of phosphoramide catalyst $\mathbf{C 1}$.

Table S6. The effect of solvent on the asymmetric Michael addition

6a $(0.05 \mathrm{mmol})$
1

${ }^{a}$ Isolated yield. ${ }^{b} \mathrm{Ee}$ and $\mathrm{Dr}$ were determined by chiral HPLC analysis. ${ }^{c} 0.025 \mathrm{~mol} / \mathrm{L},-78^{\circ} \mathrm{C}$ for $48 \mathrm{~h} .{ }^{d}$ Dr was determined by ${ }^{1} \mathrm{H}$ NMR. 


\section{General procedure for cationic $\mathrm{Au}(\mathrm{I})$ catalyzed formal allylation.}

(a) Formal allylation of diazothiooxindoles 1

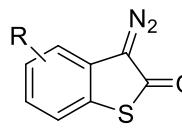

$1(0.25 \mathrm{mmol})$

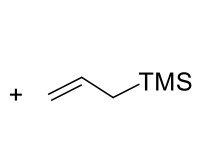

2a (5.0 equivs)
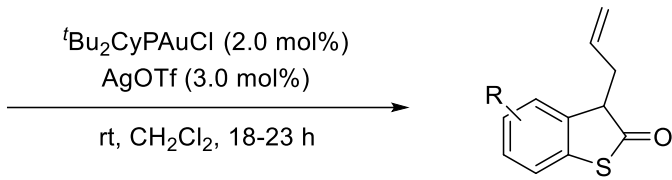

6

Under an atmosphere of nitrogen, to a Schlenk tube were added ${ }^{t} \mathrm{Bu}_{2} \mathrm{CyPAuCl}(2.3 \mathrm{mg}, 0.005$ mmol), AgOTf (2.0 mg, $0.0075 \mathrm{mmol})$, and anhydrous $\mathrm{CH}_{2} \mathrm{Cl}_{2}(2.5 \mathrm{~mL})$. The resulting mixture was stirred at room temperature for 15-20 minutes, and followed by the addition of allyltrimethylsilane $\mathbf{2 a}$ $(0.2 \mathrm{~mL}, 1.25 \mathrm{mmol})$ and diazothiooxindole $1(0.25 \mathrm{mmol})$. The reaction was kept stirring at $25{ }^{\circ} \mathrm{C}$ for about $18-23 \mathrm{~h}$ till full conversion of diazothiooxindole 1 by TLC analysis. Water $(3 \mathrm{~mL})$ and $\mathrm{CH}_{2} \mathrm{Cl}_{2}$ ( $3 \mathrm{~mL}$ ) were added and the organic layer was separated. The aqueous layer was extracted with $\mathrm{CH}_{2} \mathrm{Cl}_{2}$ $(5 \times 3 \mathrm{~mL})$, then the organic phase was combined, washed with brine $(5 \mathrm{~mL})$, dried over anhydrous $\mathrm{Na}_{2} \mathrm{SO}_{4}$ and concentrated to give the crude residue, which was purified by column chromatography eluting with EtOAc/PE (1:15, v/v) to give the desired product 6.

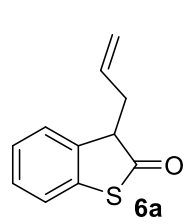

Characterization of compound 6a (yellow oil): $36.1 \mathrm{mg}, 76 \%$ yield. ${ }^{1} \mathrm{H}$ NMR (400 $\left.\mathrm{MHz} \mathrm{CDCl}_{3}\right): \delta$ 7.35-7.22 (m, 4H), 5.71-5.61 (m, 1H), 5.10-5.02 (m, 2H), $3.88(\mathrm{t}, J=$ $6.4 \mathrm{~Hz}, 1 \mathrm{H}), 2.81-2.77(\mathrm{~m}, 2 \mathrm{H}) ;{ }^{13} \mathrm{C}$ NMR (100 MHz, $\left.\mathrm{CDCl}_{3}\right): \delta 205.8,136.2,136.0$, 132.6, 128.4, 126.1, 125.0, 123.0, 118.9, 56.6, 36.7; IR (Neat): 2361, 2342, 1709, 1595, 1466, 1449, 1128, 1086, $995 \mathrm{~cm}^{-1}$; GC-MS (EI): 190 (M+, 94), 121 (100), 149 (73), 147 (57), 129 (44), 128 (38), 134 (36), 77 (36); HRMS (TOF, EI): Exact mass calcd for $\mathrm{C}_{11} \mathrm{H}_{10} \mathrm{OS}$ : 190.0452, Found: 190.0450 .

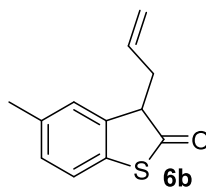

Characterization of compound $\mathbf{6 b}$ (yellow oil): $34.2 \mathrm{mg}, 67 \%$ yield. ${ }^{1} \mathrm{H}$ NMR (400 $\left.\mathrm{MHz}, \mathrm{CDCl}_{3}\right): \delta$ 7.26-7.21 (m, 1H), 7.12-7.10 (m, 2H), 5.70-5.61 (m, 1H), 5.11-5.03

$(\mathrm{m}, 2 \mathrm{H}), 3.84(\mathrm{t}, J=6.4 \mathrm{~Hz}, 1 \mathrm{H}), 2.80-2.76(\mathrm{~m}, 2 \mathrm{H}), 2.36(\mathrm{~s}, 3 \mathrm{H}) ;{ }^{13} \mathrm{C} \mathrm{NMR}(100$

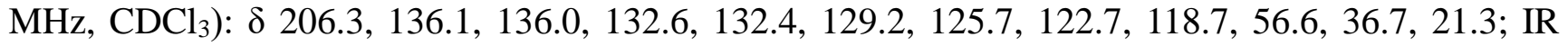
(Neat): 2361, 2342, 1713, 1639, 1472, 1142, 1086, 993, $922 \mathrm{~cm}^{-1}$; HRMS (ESI): Exact mass calcd for $\mathrm{C}_{12} \mathrm{H}_{12} \mathrm{NaOS}[\mathrm{M}+\mathrm{Na}]^{+}:$227.0501, Found: 227.0501. 
Characterization of compound 6c (yellow oil): $27.0 \mathrm{mg}, 49 \%$ yield. ${ }^{1} \mathrm{H}$ NMR $(400$
$\left.\mathrm{MHz}, \mathrm{CDCl}_{3}\right): \delta 7.20-7.18(\mathrm{~m}, 1 \mathrm{H}), 6.90(\mathrm{~d}, J=1.6 \mathrm{~Hz}, 1 \mathrm{H}), 6.78-6.75(\mathrm{~m}, 1 \mathrm{H})$, $(\mathrm{m}, 2 \mathrm{H}) ;{ }^{13} \mathrm{C} \mathrm{NMR}\left(100 \mathrm{MHz}, \mathrm{CDCl}_{3}\right): \delta 206.1,159.6,136.9,132.7,128.0,125.7,118.8,112.2,108.6$, 55.9, 55.5, 36.9; IR (Neat): 2361, 2342, 1709, 1603, 1489, 1229, 1036, 922, $646 \mathrm{~cm}^{-1}$; HRMS (ESI): Exact mass calcd for $\mathrm{C}_{12} \mathrm{H}_{12} \mathrm{NaO}_{2} \mathrm{~S}[\mathrm{M}+\mathrm{Na}]^{+}$: 243.0450, Found: 243.0440 .

Characterization of compound 6d (yellow oil): $37.5 \mathrm{mg}, 63 \%$ yield. ${ }^{1} \mathrm{H}$ NMR $(400$
$\left.\begin{array}{l}\left.\mathrm{MHz}, \mathrm{CDCl}_{3}\right): \delta 7.19-7.06(\mathrm{~m}, 1 \mathrm{H}), 6.94-6.93(\mathrm{~m}, 1 \mathrm{H}), 5.62-5.52(\mathrm{~m}, 1 \mathrm{H}), 5.04-4.97 \\ (\mathrm{~m}, 2 \mathrm{H}), 3.85(\mathrm{t}, J=6.4 \mathrm{~Hz}, 1 \mathrm{H}), 2.72-2.68(\mathrm{~m}, 2 \mathrm{H}), 2.27(\mathrm{~s}, 3 \mathrm{H}) ;{ }^{13} \mathrm{C} \mathrm{NMR}(100 \mathrm{MHz},\end{array} \mathrm{CDCl}_{3}\right): \delta 203.4,136.5,136.4,131.3,131.2,128.0,126.8,122.8,118.1,57.0,35.6$, 20.1; IR (Neat): 2361, 2342, 1717, 1642, 1560, 1445, 1161, 1036, 922, $908 \mathrm{~cm}^{-1}$; HRMS (ESI): Exact mass calcd for $\mathrm{C}_{12} \mathrm{H}_{11} \mathrm{ClNaOS}[\mathrm{M}+\mathrm{Na}]^{+}: 261.0111$, Found: 261.0101 . 


\section{(b) Formal allylation of diazooxindoles 8}

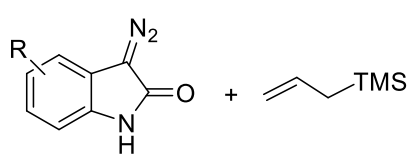

$8(0.25 \mathrm{mmol})$

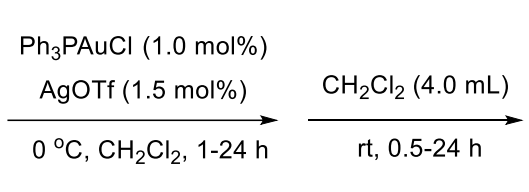

$2 \mathbf{a}$ (5.0 equivs)

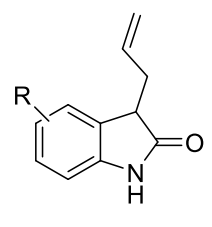

Under an atmosphere of nitrogen, to a Schlenk tube were added $\mathrm{Ph}_{3} \mathrm{PAuCl}(1.2 \mathrm{mg}, 0.0025 \mathrm{mmol})$ and $\operatorname{AgOTf}(1.0 \mathrm{mg}, 0.0038 \mathrm{mmol})$, followed by anhydrous $\mathrm{CH}_{2} \mathrm{Cl}_{2}(4.0 \mathrm{~mL})$. The resulting mixture was stirred at room temperature for 15-20 minutes, and then cooled down to $0{ }^{\circ} \mathrm{C}$, followed by the addition of allyltrimethylsilane $\mathbf{2 a}(0.2 \mathrm{~mL}, 1.25 \mathrm{mmol})$ and diazooxindole $8(0.25 \mathrm{mmol})$. The reaction was kept stirring at $0{ }^{\circ} \mathrm{C}$ for about $1-24 \mathrm{~h}$ till full conversion of diazooxindole 8 by TLC analysis. After the anhydrous $\mathrm{CH}_{2} \mathrm{Cl}_{2}$ and excess silane $\mathbf{2 a}$ were removed under reduced pressure, ordinary $\mathrm{CH}_{2} \mathrm{Cl}_{2}$ (not anhydrous, $4 \mathrm{~mL}$ ) was added. The solution was kept stirring at $25{ }^{\circ} \mathrm{C}$ for about 0.5-24 $\mathrm{h}$ till the ring-opening process finished by TLC analysis. Water $(3 \mathrm{~mL})$ and $\mathrm{CH}_{2} \mathrm{Cl}_{2}(3 \mathrm{~mL})$ were added and the organic layer was separated. The aqueous layer was extracted with $\mathrm{CH}_{2} \mathrm{Cl}_{2}(5 \times 3$ $\mathrm{mL})$, then the organic phase was combined, washed with brine $(5 \mathrm{~mL})$, dried over anhydrous $\mathrm{Na}_{2} \mathrm{SO}_{4}$ and concentrated to give the crude residue, which was purified by column chromatography eluting with EtOAc/PE (1:5, v/v) to give the desired product 7.

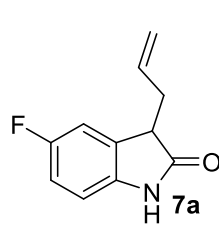

Characterization of compound 7a (reddish-brown solid): $37.7 \mathrm{mg}, 79 \%$ yield. $\mathrm{Mp}$ 75-76 ${ }^{\circ} \mathrm{C} .{ }^{1} \mathrm{H}$ NMR $\left(400 \mathrm{MHz}, \mathrm{CDCl}_{3}\right): \delta 9.46$ (s, 1H), 6.99-6.80 (m, 3H), 5.76-5.69

$(\mathrm{m}, 1 \mathrm{H}), 5.13-5.04(\mathrm{~m}, 2 \mathrm{H}), 3.52-3.49(\mathrm{~m}, 1 \mathrm{H}), 2.83-2.77(\mathrm{~m}, 1 \mathrm{H}), 2.59-2.51(\mathrm{~m}, 1 \mathrm{H})$;

${ }^{13} \mathrm{C}$ NMR $\left(100 \mathrm{MHz}, \mathrm{CDCl}_{3}\right): \delta 180.2,158.9\left(\mathrm{~d}, J_{\mathrm{C}-\mathrm{F}}=238.0 \mathrm{~Hz}\right), 137.6(\mathrm{~d}, J=2.0$ $\mathrm{Hz}), 133.4,130.8\left(\mathrm{~d}, J_{\mathrm{C}-\mathrm{F}}=8.0 \mathrm{~Hz}\right), 118.5,114.3\left(\mathrm{~d}, J_{\mathrm{C}-\mathrm{F}}=24.0 \mathrm{~Hz}\right), 112.4\left(\mathrm{~d}, J_{\mathrm{C}-\mathrm{F}}=25.0 \mathrm{~Hz}\right), 110.3$ $\left(\mathrm{d}, J_{\mathrm{C}-\mathrm{F}}=8.0 \mathrm{~Hz}\right), 46.2\left(\mathrm{~d}, J_{\mathrm{C}-\mathrm{F}}=2.0 \mathrm{~Hz}\right), 34.6 ;{ }^{19} \mathrm{~F} \mathrm{NMR}\left(376 \mathrm{MHz}, \mathrm{CDCl}_{3}\right): \delta-120.87$; IR (Neat): 2361, 1709, 1485, 1312, 1254, 1198, 1132, 1067, $922 \mathrm{~cm}^{-1}$; HRMS (ESI): Exact mass calcd for $\mathrm{C}_{11} \mathrm{H}_{10} \mathrm{FNNaO}[\mathrm{M}+\mathrm{Na}]^{+}: 214.0639$, Found: 214.0632.

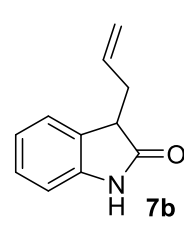

Characterization of compound $7 \mathbf{7 b}^{8}$ (white powder): $32.4 \mathrm{mg}, 75 \%$ yield. ${ }^{1} \mathrm{H}$ NMR (400 $\left.\mathrm{MHz}, \mathrm{CDCl}_{3}\right): \delta 9.10(\mathrm{~s}, 1 \mathrm{H}), 7.27-7.19(\mathrm{~m}, 2 \mathrm{H}), 7.03-6.99(\mathrm{~m}, 1 \mathrm{H}), 6.92(\mathrm{~d}, J=7.6$ $\mathrm{Hz}, 1 \mathrm{H}), 5.81-5.74(\mathrm{~m}, 1 \mathrm{H}), 5.15-5.05(\mathrm{~m}, 2 \mathrm{H}), 3.55-3.52(\mathrm{~m}, 1 \mathrm{H}), 2.86-2.81(\mathrm{~m}, 1 \mathrm{H})$, 2.64-2.56 (m, 1H); ${ }^{13} \mathrm{C}$ NMR (100 MHz, $\left.\mathrm{CDCl}_{3}\right): \delta 180.1,141.6,133.9,129.3,128.0$, 124.4, 122.2, 118.1, 109.8, 45.8, 34.8. 


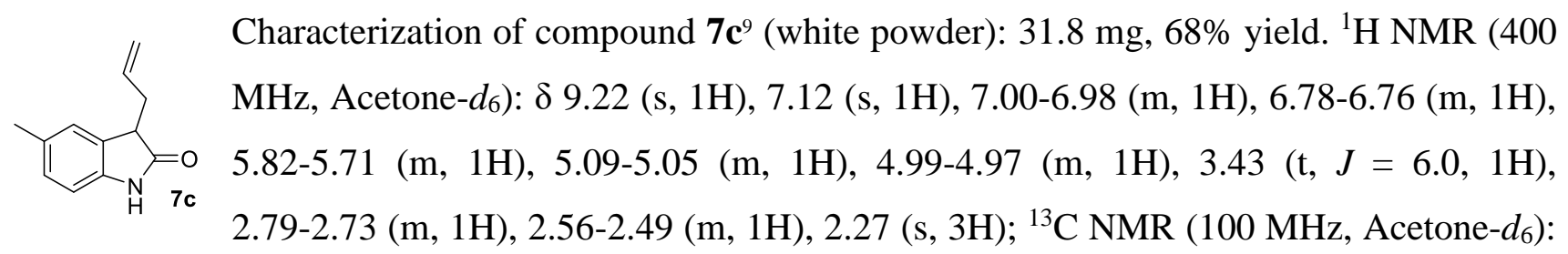
$\delta 178.9,141.2,135.5,131.4,130.4,128.8,125.9,117.7,109.8,46.0,35.3,21.1$.

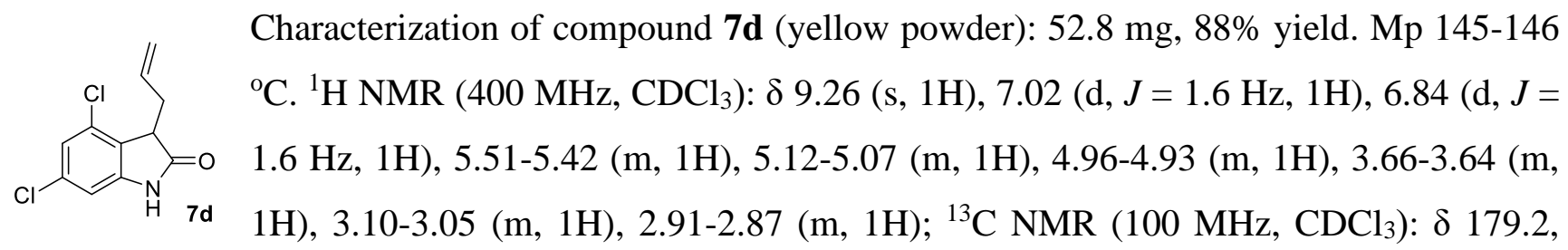
143.8, 134.6, 131.7, 131.3, 124.5, 122.7, 118.9, 109.1, 46.2, 31.9; IR (Neat): 2361, 2342, 1719, 1614, 1586, 1441, 1316, 1080, 982, $922 \mathrm{~cm}^{-1}$; HRMS (ESI): Exact mass calcd for $\mathrm{C}_{11} \mathrm{H}_{9} \mathrm{Cl}_{2} \mathrm{NNaO}[\mathrm{M}+\mathrm{Na}]^{+}$: 263.9953, Found: 263.9947. 


\section{General procedure for tandem formal allylation/Michael addition sequence}

(a) Tandem reaction based on diazothiooxindoles 1

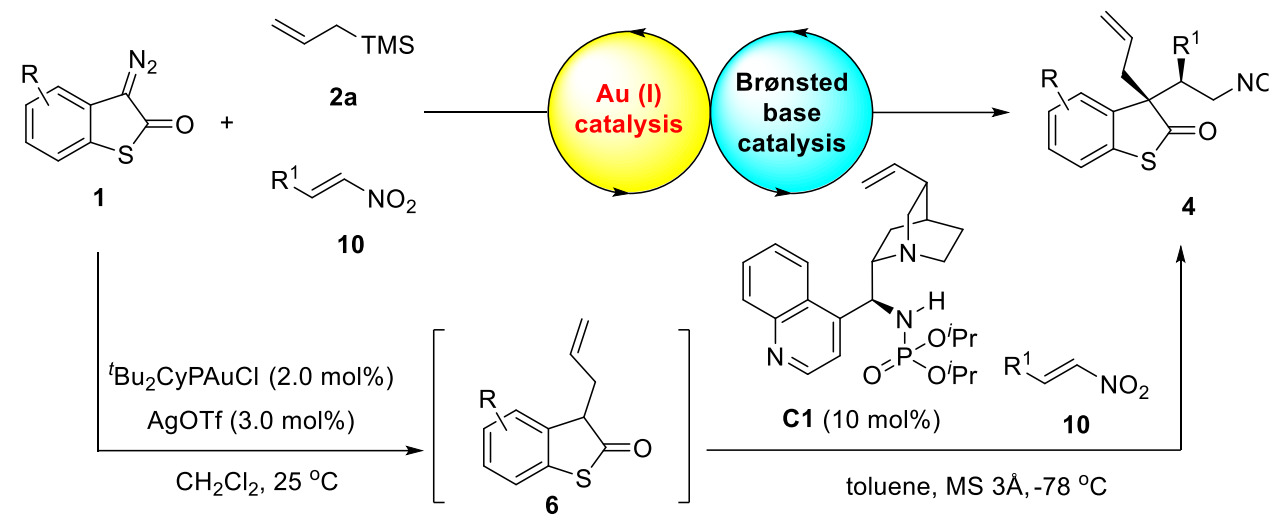

Under an atmosphere of nitrogen, to a Schlenk tube were added ${ }^{t} \mathrm{Bu}_{2} \mathrm{CyPAuCl}(2.3 \mathrm{mg}, 0.005$ mmol), AgOTf (2.0 mg, $0.0075 \mathrm{mmol})$, and anhydrous $\mathrm{CH}_{2} \mathrm{Cl}_{2}(2.5 \mathrm{~mL})$. The resulting mixture was stirred at room temperature for 15-20 minutes, and followed by the addition of allyltrimethylsilane $2 \mathbf{a}$ $(0.2 \mathrm{~mL}, 1.25 \mathrm{mmol})$ and diazothiooxindole $1(0.25 \mathrm{mmol})$. The reaction was kept stirring at $25{ }^{\circ} \mathrm{C}$ for about $18 \mathrm{~h}$ till full conversion of diazothiooxindole 1 by TLC analysis. Then $\mathrm{CH}_{2} \mathrm{Cl}_{2}$ was removed under reduced pressure, and the residue was directly subjected to a short column chromatography to remove ${ }^{t} \mathrm{Bu}_{2} \mathrm{CyPAuOTf}$ using an eluent of EtOAc/PE (1:15, v/v). After concentration, toluene $(10 \mathrm{~mL})$, powdered MS $3 \AA$ (250 mg) and chiral catalyst C1 (11.4 mg, $0.025 \mathrm{mmol})$ were added successively. After cooling down to $-78{ }^{\circ} \mathrm{C}$ for 30 minutes, the nitroolefins $\mathbf{1 0}(0.25 \mathrm{mmol})$ was added. The resulting mixture was stirred till full conversion of $\mathbf{6}$ by TLC analysis. Then the solvent was carefully removed under reduced pressure. To determine the diastereoselectivity of product, the residue was first dissolved in $\mathrm{CDCl}_{3}$, and some samples were taken for NMR analysis. Then the sample for analysis and the rest of the product were recombined for column chromatographic purification using EtOAc/PE (from 1:50 to 1:10, v/v) as the eluent.

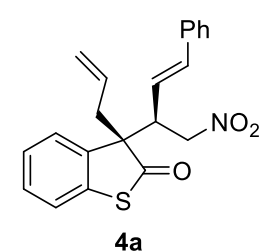

Product 4a was obtained in $59.3 \mathrm{mg}$ (65\% yield) as colorless oil. ${ }^{1} \mathrm{H}$ NMR analysis revealed that the dr value was 9:1. HPLC analysis: [Chiralcel IA, 5\% ${ }^{i} \mathrm{PrOH} /$ hexane, $1.0 \mathrm{~mL} / \mathrm{min}, 254 \mathrm{~nm}$; major diastereomer: $\mathrm{t}_{\mathrm{r}}($ minor $)=8.59 \mathrm{~min}, \mathrm{t}_{\mathrm{r}}($ major $)=11.31$ min; minor diastereomer: $\mathrm{t}_{\mathrm{r}}($ minor $)=9.28 \mathrm{~min}, \mathrm{t}_{\mathrm{r}}($ major $\left.)=7.41 \mathrm{~min}\right]$ gave the isomeric composition of major diastereomer: $92 \%$ ee; $[\alpha]^{25} \mathrm{D}=+2.5\left(\mathrm{c}=0.50, \mathrm{CH}_{2} \mathrm{Cl}_{2}\right) .{ }^{1} \mathrm{H} \mathrm{NMR}(400$ $\left.\mathrm{MHz}, \mathrm{CDCl}_{3}\right): \delta$ 7.41-7.26 (m, 9H), $6.58(\mathrm{~d}, J=16.0 \mathrm{~Hz}, 1 \mathrm{H}), 6.41-6.34(\mathrm{~m}, 1 \mathrm{H}), 5.33-5.31(\mathrm{~m}, 1 \mathrm{H})$, 
4.99-4.93 (m, 2H), 4.39 (t, $J=11.6 \mathrm{~Hz}, 1 \mathrm{H}), 4.09$ (dd, $J=12.0,3.6 \mathrm{~Hz}, 1 \mathrm{H}), 3.49-3.48$ (m, 1H), 2.83, $2.57(\mathrm{ABd}, J=13.6, J=7.6 \mathrm{~Hz}, 2 \mathrm{H}) ;{ }^{13} \mathrm{C} \mathrm{NMR}\left(125 \mathrm{MHz}, \mathrm{CDCl}_{3}\right): \delta 206.5,137.3,137.0,136.0$, $135.7,130.2$, 129.4, 128.6, 128.3, 127.2, 126.8, 124.4, 123.4, 121.7, 120.5, 75.9, 64.1, 51.7, 42.9; IR (ATR): 1709, 1558, 1375, 1248, 1204, 1049, 910, 880, $764 \mathrm{~cm}^{-1}$; HRMS (ESI): Exact mass calcd for $\mathrm{C}_{21} \mathrm{H}_{19} \mathrm{NNaO}_{3} \mathrm{~S}[\mathrm{M}+\mathrm{Na}]^{+}:$388.0978, Found: 388.0985.

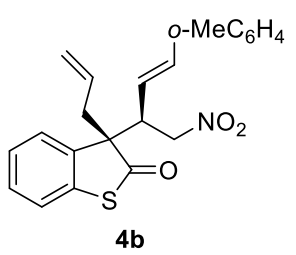

Product 4b was obtained in $60.6 \mathrm{mg}$ (64\% yield) as colorless oil. ${ }^{1} \mathrm{H}$ NMR analysis revealed that the dr value was 10:1. HPLC analysis: [Chiralcel OZ-H, 3\% ${ }^{i} \mathrm{PrOH} /$ hexane, $1.0 \mathrm{~mL} / \mathrm{min}, 254 \mathrm{~nm}$; major diastereomer: $\mathrm{t}_{\mathrm{r}}$ (major) $=7.66 \mathrm{~min}, \mathrm{t}_{\mathrm{r}}$ $($ minor $)=9.50 \mathrm{~min}]$ gave the isomeric composition of major diastereomer: $90 \%$ ee; $[\alpha]^{25} \mathrm{D}=+3.5\left(\mathrm{c}=0.50, \mathrm{CH}_{2} \mathrm{Cl}_{2}\right) .{ }^{1} \mathrm{H}$ NMR $\left(400 \mathrm{MHz}, \mathrm{CDCl}_{3}\right): \delta$ 7.42-7.17 (m, 8H), $6.77(\mathrm{~d}, J=15.6$ $\mathrm{Hz}, 1 \mathrm{H}), 6.19$ (dd, $J=15.6 \mathrm{~Hz}, 9.6 \mathrm{~Hz}, 1 \mathrm{H}), 5.36-5.34(\mathrm{~m}, 1 \mathrm{H}), 5.00-4.94(\mathrm{~m}, 2 \mathrm{H}), 4.40(\mathrm{t}, J=11.6$ $\mathrm{Hz}, 1 \mathrm{H}), 4.14(\mathrm{dd}, J=12.0,3.2 \mathrm{~Hz}, 1 \mathrm{H}), 3.53-3.50(\mathrm{~m}, 1 \mathrm{H}), 2.85,2.59(\mathrm{ABd}, J=13.6, J=8.0 \mathrm{~Hz}$, 2H), $2.32(\mathrm{~s}, 3 \mathrm{H}) ;{ }^{13} \mathrm{C} \mathrm{NMR}\left(125 \mathrm{MHz}, \mathrm{CDCl}_{3}\right): \delta 206.4,137.3,135.7,135.6,135.4,135.2,130.2$, $130.1,129.3,128.2,127.1,126.3,126.2,124.4,123.4,123.2,120.5,76.0,64.1,51.8,42.8,19.8$; IR (ATR): 1703, 1562, 1555, 1377, 1339, 1074, 972, 895, $750 \mathrm{~cm}^{-1}$; HRMS (ESI): Exact mass calcd for $\mathrm{C}_{22} \mathrm{H}_{21} \mathrm{NNaO}_{3} \mathrm{~S}[\mathrm{M}+\mathrm{Na}]^{+}:$402.1134, Found: 402.1133 .

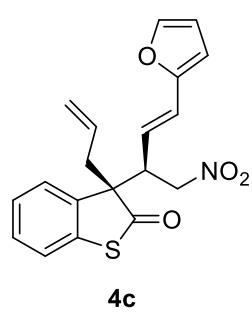

Product 4c was obtained in $54.1 \mathrm{mg}$ (61\% yield) as colorless oil. ${ }^{1} \mathrm{H}$ NMR analysis revealed that the dr value was >20:1. HPLC analysis: [Chiralcel IA, 3\% ${ }^{i} \mathrm{PrOH} / \mathrm{hexane}, 1.0 \mathrm{~mL} / \mathrm{min}, 254 \mathrm{~nm}$; major diastereomer: $\mathrm{t}_{\mathrm{r}}$ (major) $=20.53 \mathrm{~min}, \mathrm{t}_{\mathrm{r}}$ $($ minor $)=11.39 \mathrm{~min}]$ gave the isomeric composition of major diastereomer: $86 \%$ ee; $[\alpha]^{25} \mathrm{D}=+6.9\left(\mathrm{c}=0.50, \mathrm{CH}_{2} \mathrm{Cl}_{2}\right) .{ }^{1} \mathrm{H}$ NMR $\left(400 \mathrm{MHz}, \mathrm{CDCl}_{3}\right): \delta 7.39-7.36(\mathrm{~m}, 5 \mathrm{H})$, 6.38-6.28 (m, 4H), 5.33-5.31 (m, 1H), 4.99-4.92 (m, 2H), 4.37 (ABd, J=12.0, 11.2 Hz, 1H), 4.09 (dd, $J=12.0,3.2 \mathrm{~Hz}, 1 \mathrm{H}), 3.45-3.40(\mathrm{~m}, 1 \mathrm{H}), 2.84,2.55(\mathrm{ABd}, J=13.6, \mathrm{~Hz}, 2 \mathrm{H}) ;{ }^{13} \mathrm{C} \mathrm{NMR}(100 \mathrm{MHz}$, $\left.\mathrm{CDCl}_{3}\right): \delta 206.2,151.5,142.6,137.3,135.8,130.2,129.3,127.1,124.9,124.4,123.4,120.4,120.1$, 111.3, 109.2, 75.8, 64.1, 51.5, 42.8; IR (ATR): 1703, 1697, 1555, 1377, 1015, 966, 927, $745 \mathrm{~cm}^{-1}$; HRMS (ESI): Exact mass calcd for $\mathrm{C}_{19} \mathrm{H}_{17} \mathrm{NNaO}_{4} \mathrm{~S}$ [M+Na] $]^{+}$378.0770, Found: 378.0760. 


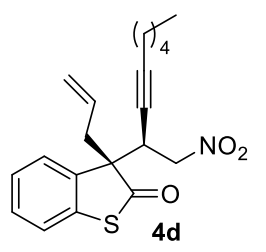

Product 4d was obtained in $60.7 \mathrm{mg}$ (68\% yield) as colorless oil. ${ }^{1} \mathrm{H}$ NMR analysis revealed that the $\mathrm{dr}$ value was 5:1. HPLC analysis: [Chiralcel AS-H, 0.5\% ${ }^{i} \mathrm{PrOH} / \mathrm{hexane}, 1.0 \mathrm{~mL} / \mathrm{min}, 230 \mathrm{~nm}$; major diastereomer: $\mathrm{t}_{\mathrm{r}}$ (major) $=15.80 \mathrm{~min}, \mathrm{t}_{\mathrm{r}}$ $($ minor $)=17.68 \mathrm{~min}]$ gave the isomeric composition of major diastereomer: $88 \%$ ee; $[\alpha]^{25}{ }_{\mathrm{D}}=-8.0\left(\mathrm{c}=0.50, \mathrm{CHCl}_{3}\right) .{ }^{1} \mathrm{H} \mathrm{NMR}\left(400 \mathrm{MHz}, \mathrm{CDCl}_{3}\right): \delta$ 7.38-7.30 (m, 4H), 5.39-5.37 (m, $\left.1 \mathrm{H}\right)$, 5.06-4.98 (m, 1H), $4.43(\mathrm{ABd}, J=12.4,10.8 \mathrm{~Hz}, 1 \mathrm{H}), 4.18$ (dd, $J=12.4,3.6 \mathrm{~Hz}, 1 \mathrm{H}), 3.75-3.71(\mathrm{~m}$, $1 \mathrm{H}), 3.02-2.96(\mathrm{~m}, 1 \mathrm{H}), 2.78-2.72(\mathrm{~m}, 1 \mathrm{H}), 2.19(\mathrm{td}, J=7.2,2.4 \mathrm{~Hz}, 2 \mathrm{H}), 1.49-1.35(\mathrm{~m}, 2 \mathrm{H})$, 1.34-1.31 (m, 4H), 0.92-0.88 (m, 3H); ${ }^{13} \mathrm{C}$ NMR (100 MHz, $\left.\mathrm{CDCl}_{3}\right): \delta 204.6,136.5,135.6,130.4$, 129.4, 126.7, 124.9, 123.3, 120.5, 88.7, 75.6, 72.4, 62.9, 41.0, 39.8, 30.9, 28.1, 22.1, 18.7, 14.0; IR (ATR): 1711, 1558, 1468, 1449, 1375, 928, 905, $750 \mathrm{~cm}^{-1}$; HRMS (ESI): Exact mass calcd for $\mathrm{C}_{20} \mathrm{H}_{23} \mathrm{NNaO}_{3} \mathrm{~S}[\mathrm{M}+\mathrm{Na}]^{+}:$380.1291, Found: 380.1292 .

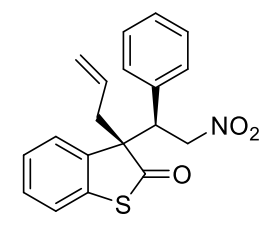

$4 e$

Product 4e was obtained in $52.5 \mathrm{mg}$ (62\% yield) as colorless oil. ${ }^{1} \mathrm{H}$ NMR analysis revealed that the dr value was >20:1. HPLC analysis: [Chiralcel AD-H, 3\% ${ }^{i} \mathrm{PrOH} /$ hexane, $1.0 \mathrm{~mL} / \mathrm{min}, 230 \mathrm{~nm}$; major diastereomer: $\mathrm{t}_{\mathrm{r}}$ (major) $=16.34 \mathrm{~min}, \mathrm{t}_{\mathrm{r}}$ $($ minor $)=14.82 \mathrm{~min}]$ gave the isomeric composition of major diastereomer: $93 \%$ ee; $[\alpha]^{25}{ }_{\mathrm{D}}=+12.4\left(\mathrm{c}=0.50, \mathrm{CH}_{2} \mathrm{Cl}_{2}\right) .{ }^{1} \mathrm{H} \mathrm{NMR}\left(400 \mathrm{MHz}, \mathrm{CDCl}_{3}\right): \delta 7.39-7.31(\mathrm{~m}, 2 \mathrm{H})$, 7.30-7.27 (m, 1H), 7.26-7.21 (m, 2H), 7.20-7.18 (m, 2H), 6.94-6.91 (m, 2H), 5.32-5.25 (m, 1H), 4.98-4.89 (m, 3H), $4.78(\mathrm{ABd}, J=13.2,4.4 \mathrm{~Hz}, 1 \mathrm{H}), 4.01(\mathrm{dd}, J=11.6,4.4 \mathrm{~Hz}, 1 \mathrm{H}), 2.69(\mathrm{dt}, J=6.8$, $1.2 \mathrm{~Hz}, 2 \mathrm{H}) ;{ }^{13} \mathrm{C}$ NMR $\left(100 \mathrm{MHz}, \mathrm{CDCl}_{3}\right): \delta 206.4,136.8,136.4,133.6,130.4,129.4,129.3,128.5$, 128.4, 126.6, 124.9, 123.3, 120.3, 75.6, 65.1, 52.1, 41.1; IR (ATR): 1705, 1557, 1449, 1377, 1092, 991, 928, 746, $702 \mathrm{~cm}^{-1}$; HRMS (ESI): Exact mass calcd for $\mathrm{C}_{19} \mathrm{H}_{17} \mathrm{NNaO}_{3} \mathrm{~S}[\mathrm{M}+\mathrm{Na}]^{+}: 362.0821$, Found: 362.0813.

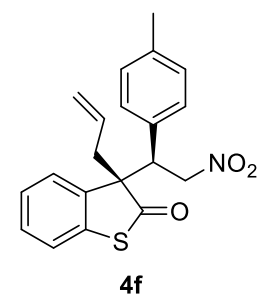

Product $4 \mathbf{f}$ was obtained in $53.8 \mathrm{mg}$ (61\% yield) as colorless oil. ${ }^{1} \mathrm{H}$ NMR analysis revealed that the $\mathrm{dr}$ value was >20:1. HPLC analysis: [Chiralcel AD-H, 3\% ${ }^{i} \mathrm{PrOH} /$ hexane, $1.0 \mathrm{~mL} / \mathrm{min}, 230 \mathrm{~nm}$; major diastereomer: $\mathrm{t}_{\mathrm{r}}$ (major) $=14.98 \mathrm{~min}, \mathrm{t}_{\mathrm{r}}$ $($ minor $)=14.19 \mathrm{~min}]$ gave the isomeric composition of major diastereomer: $92 \%$ ee; $[\alpha]^{25} \mathrm{D}=-7.1\left(\mathrm{c}=0.50, \mathrm{CH}_{2} \mathrm{Cl}_{2}\right) .{ }^{1} \mathrm{H}$ NMR $\left(400 \mathrm{MHz}, \mathrm{CDCl}_{3}\right): \delta 7.37-7.21(\mathrm{~m}, 3 \mathrm{H})$, 7.20-7.19 (m, 1H), 7.01 (d, $J=8.0 \mathrm{~Hz} 1 \mathrm{H}), 6.83-6.81(\mathrm{~m}, 2 \mathrm{H}), 5.28-5.24(\mathrm{~m}, 1 \mathrm{H})$, 4.97-4.86 (m, 3H), $4.73(\mathrm{ABd}, J=12.8,4.0 \mathrm{~Hz}, 1 \mathrm{H}), 3.96(\mathrm{dd}, J=11.6,4.0 \mathrm{~Hz}, 1 \mathrm{H}), 2.68-2.66(\mathrm{~m}, 2 \mathrm{H}), 2.28(\mathrm{~s}, 3 \mathrm{H}) ;{ }^{13} \mathrm{C}$ NMR (100 MHz, $\left.\mathrm{CDCl}_{3}\right) \delta 206.4,138.3,137.0,136.4,130.5,130.4,129.2,129,1,126.6,124.9,123.3$, 
120.2, 75.7, 65.1, 51.9, 41.2, 21.1; IR (ATR): 1703, 1556, 1447, 1377, 1338, 1104, 925, 829, $752 \mathrm{~cm}^{-1}$; HRMS (ESI): Exact mass calcd for $\mathrm{C}_{20} \mathrm{H}_{19} \mathrm{NNaO}_{3} \mathrm{~S}[\mathrm{M}+\mathrm{Na}]^{+}$: 376.0978, Found: 376.0980 .

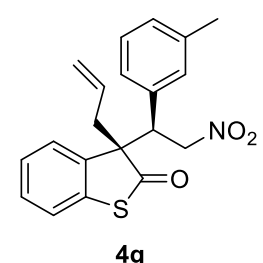

Product $4 \mathrm{~g}$ was obtained in $54.7 \mathrm{mg}$ (62\% yield) as colorless oil. ${ }^{1} \mathrm{H}$ NMR analysis revealed that the $\mathrm{dr}$ value was >20:1. HPLC analysis: [Chiralcel OD-H, 2\% ${ }^{i} \mathrm{PrOH} / \mathrm{hexane}, 1.0 \mathrm{~mL} / \mathrm{min}, 230 \mathrm{~nm}$; major diastereomer: $\mathrm{t}_{\mathrm{r}}$ (major) $=16.86 \mathrm{~min}, \mathrm{t}_{\mathrm{r}}$ $($ minor $)=23.10 \mathrm{~min}]$ gave the isomeric composition of major diastereomer: $93 \%$ ee; $[\alpha]^{25} \mathrm{D}=-4.7\left(\mathrm{c}=0.50, \mathrm{CH}_{2} \mathrm{Cl}_{2}\right) .{ }^{1} \mathrm{H}$ NMR $\left(400 \mathrm{MHz}, \mathrm{CDCl}_{3}\right): \delta$ 7.36-7.30 $(\mathrm{m}, 3 \mathrm{H}), 7.20-7.06(\mathrm{~m}, 3 \mathrm{H})$, 6.73-6.70 (m, 2H), 5.30-5.25 (m, 1H), 4.98-4.87 (m, 3H), 4.76 (ABd, $J=12.8,4.0 \mathrm{~Hz}, 1 \mathrm{H}), 3.96$ (dd, $J=11.2,4.0 \mathrm{~Hz}, 1 \mathrm{H}), 2.68(\mathrm{dd}, J=7.2,1.2 \mathrm{~Hz}, 2 \mathrm{H}), 2.22(\mathrm{~s}, 3 \mathrm{H}) ;{ }^{13} \mathrm{C} \mathrm{NMR}\left(100 \mathrm{MHz}, \mathrm{CDCl}_{3}\right) \delta$ 206.4, 137.9, 137.0, 136.4, 133.5, 130.5, 130.2, 129,2, 128.2, 126.5, 126.3, 124.9, 123.2, 120.3, 75.6, 65.2, 52.1, 41.0, 21.4; IR (ATR): 1701, 1555, 1447, 1377, 1208, 1101, 928, 804, $750 \mathrm{~cm}^{-1}$; HRMS (ESI): Exact mass calcd for $\mathrm{C}_{20} \mathrm{H}_{19} \mathrm{NNaO}_{3} \mathrm{~S}$ [M+Na] $]^{+}: 376.0978$, Found: 376.0975 .

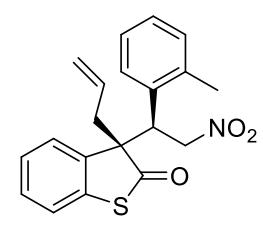

$4 \mathbf{h}$

Product 4h was obtained in $52.0 \mathrm{mg}$ (59\% yield) as colorless oil. ${ }^{1} \mathrm{H}$ NMR analysis revealed that the dr value was >20:1. HPLC analysis: [Chiralcel AD-H, 0.5\% ${ }^{i} \mathrm{PrOH} /$ hexane, $1.0 \mathrm{~mL} / \mathrm{min}, 230 \mathrm{~nm}$; major diastereomer: $\mathrm{t}_{\mathrm{r}}$ (major) $=31.23 \mathrm{~min}, \mathrm{t}_{\mathrm{r}}$ $($ minor $)=36.71 \mathrm{~min}]$ gave the isomeric composition of major diastereomer: $93 \%$ ee; $[\alpha]^{25}{ }_{\mathrm{D}}=-8.9\left(\mathrm{c}=0.50, \mathrm{CH}_{2} \mathrm{Cl}_{2}\right) .{ }^{1} \mathrm{H} \mathrm{NMR}\left(400 \mathrm{MHz}, \mathrm{CDCl}_{3}\right): \delta$ 7.34-7.32 (m, 2H), 7.18-7.05 (m, 4H), 6.86-6.84 (m, 1H), 6.74-6.72 (m, 1H), 5.26-5.24 (m, 3H), 5.03-4.88 (m, 4H), $4.36(\mathrm{dd}, J=11.2,4.4$ $\mathrm{Hz}, 1 \mathrm{H}), 2.78-2.76(\mathrm{~m}, 2 \mathrm{H}), 2.06(\mathrm{~s}, 3 \mathrm{H}) ;{ }^{13} \mathrm{C} \mathrm{NMR}\left(100 \mathrm{MHz}, \mathrm{CDCl}_{3}\right) \delta 206.7,138.7,136.7,136.1$, 132.7, 131.0, 130.5, 129,2, 128.2, 126.7, 126.2, 126.0, 125.4, 123.1, 120.3, 75.9, 65.1, 44.6, 39.1, 19.5; IR (ATR): 1701, 1557, 1447, 1377, 1221, 1101, 926, 908, $748 \mathrm{~cm}^{-1}$; HRMS (ESI): Exact mass calcd for $\mathrm{C}_{20} \mathrm{H}_{19} \mathrm{NNaO}_{3} \mathrm{~S}[\mathrm{M}+\mathrm{Na}]^{+}:$376.0978, Found: 376.0983 .

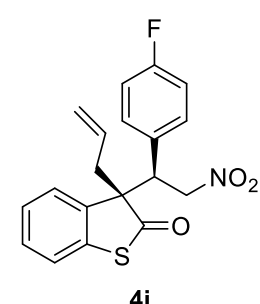

$4 \mathbf{i}$

Product 4i was obtained in $60.7 \mathrm{mg}$ (68\% yield) as colorless oil. ${ }^{1} \mathrm{H}$ NMR analysis revealed that the dr value was 6:1. HPLC analysis: [Chiralcel AD-H, 3\% ${ }^{i} \mathrm{PrOH} /$ hexane, $1.0 \mathrm{~mL} / \mathrm{min}, 230 \mathrm{~nm}$; major diastereomer: $\mathrm{t}_{\mathrm{r}}$ (major) $=21.24 \mathrm{~min}, \mathrm{t}_{\mathrm{r}}$ $($ minor $)=18.78 \mathrm{~min}]$ gave the isomeric composition of major diastereomer: $91 \%$ ee; $[\alpha]^{25} \mathrm{D}=-6.0\left(\mathrm{c}=0.50, \mathrm{CHCl}_{3}\right) .{ }^{1} \mathrm{H} \mathrm{NMR}\left(400 \mathrm{MHz}, \mathrm{CDCl}_{3}\right): \delta 7.38-7.35(\mathrm{~m}, 2 \mathrm{H})$, 7.32-7.31 (m, 1H), 7.24-7.23 (m, 1H), $6.89(\mathrm{~d}, J=6.8 \mathrm{~Hz}, 4 \mathrm{H}), 5.30-5.26(\mathrm{~m}, 1 \mathrm{H}), 4.99-4.83(\mathrm{~m}, 3 \mathrm{H})$, $4.77(\mathrm{ABd}, J=12.8,4.4 \mathrm{~Hz}, 1 \mathrm{H}), 4.01(\mathrm{dd}, J=11.6,4.4 \mathrm{~Hz}, 1 \mathrm{H}), 2.69-2.66(\mathrm{~m}, 2 \mathrm{H}) ;{ }^{13} \mathrm{C}$ NMR $(100$ 
$\left.\mathrm{MHz}, \mathrm{CDCl}_{3}\right): \delta 206.4,162.7\left(\mathrm{~d}, J_{\mathrm{F}-\mathrm{C}}=247.0 \mathrm{~Hz}\right), 136.5\left(\mathrm{~d}, J_{\mathrm{F}-\mathrm{C}}=9.0 \mathrm{~Hz}\right), 131.0\left(\mathrm{~d}, J_{\mathrm{F}-\mathrm{C}}=9.0 \mathrm{~Hz}\right)$, $130.2,129.4,129.3\left(\mathrm{~d}, J_{\mathrm{F}-\mathrm{C}}=3.0 \mathrm{~Hz}\right), 126.7,124.7,123.4,120.5,115.5,115.3$, 75.6, 65.1, 51.4, 41.2; ${ }^{19} \mathrm{~F}$ NMR (376 MHz, $\mathrm{CDCl}_{3}$ ): $\delta$-113.06; IR (ATR): 1701, 1591, 1447, 1377, 1228, 1103, 927, 840, $754 \mathrm{~cm}^{-1}$; HRMS (ESI): Exact mass calcd for $\mathrm{C}_{19} \mathrm{H}_{16} \mathrm{FNNaO}_{3} \mathrm{~S}[\mathrm{M}+\mathrm{Na}]^{+}:$380.0727, Found: 380.0718 .

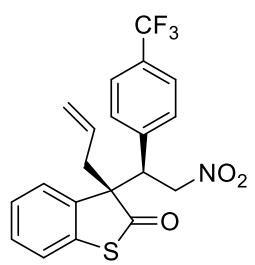

4j

Product 4j was obtained in $67.2 \mathrm{mg}$ (66\% yield) as colorless oil. ${ }^{1} \mathrm{H}$ NMR analysis revealed that the dr value was 12:1. HPLC analysis: [Chiralcel AD-H, 3\% ${ }^{i} \mathrm{PrOH} /$ hexane, $1.0 \mathrm{~mL} / \mathrm{min}, 230 \mathrm{~nm}$; major diastereomer: $\mathrm{t}_{\mathrm{r}}$ (major) $=19.40 \mathrm{~min}, \mathrm{t}_{\mathrm{r}}$ $($ minor $)=16.48 \mathrm{~min}]$ gave the isomeric composition of major diastereomer: $84 \%$ ee; $[\alpha]^{25} \mathrm{D}=-1.9\left(\mathrm{c}=0.50, \mathrm{CHCl}_{3}\right) .{ }^{1} \mathrm{H}$ NMR $\left(400 \mathrm{MHz}, \mathrm{CDCl}_{3}\right): \delta 7.47(\mathrm{~d}, J=8.0 \mathrm{~Hz}$, 2H), 7.40-7.38 (m, 2H), 7.33-7.31 (m, 1H), 7.28-7.26 (m, 1H), $7.07(\mathrm{~d}, J=8.0 \mathrm{~Hz}, 2 \mathrm{H})$, 5.34-5.23 (m, 1H), 4.99-4.88 (m, 3H), 4.77 (ABd, $J=12.8,4.0 \mathrm{~Hz}, 1 \mathrm{H}), 4.09$ (dd, $J=11.6,4.4 \mathrm{~Hz}, 1 \mathrm{H}), 2.68-2.66$ $(\mathrm{m}, 2 \mathrm{H}) ;{ }^{13} \mathrm{C}$ NMR $\left(125 \mathrm{MHz}, \mathrm{CDCl}_{3}\right): \delta 206.2,137.7,136.3\left(\mathrm{~d}, J_{\mathrm{F}-\mathrm{C}}=7.5 \mathrm{~Hz}\right), 130.7\left(\mathrm{q}, J_{\mathrm{F}-\mathrm{C}}=33.8\right.$ Hz), 129.9, 129.8, 129.7, 129.6, 126.9, 125.3, 124.7, 123.7 (q, $\left.J_{\mathrm{F}-\mathrm{C}}=271.3 \mathrm{~Hz}\right), 120.7,75.3,64.8$, 51.8, 41.4; ${ }^{19} \mathrm{~F}$ NMR (376 MHz, $\mathrm{CDCl}_{3}$ ): $\delta$-62.78; IR (ATR): 1703, 1558, 1449, 1377, 1327, 1118, 930, 849, $754 \mathrm{~cm}^{-1}$; HRMS (ESI): Exact mass calcd for $\mathrm{C}_{20} \mathrm{H}_{16} \mathrm{~F}_{3} \mathrm{NNaO}_{3} \mathrm{~S}[\mathrm{M}+\mathrm{Na}]^{+}:$430.0695, Found: 430.0691 .

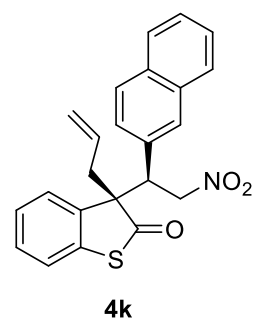

Product 4k was obtained in $60.3 \mathrm{mg}$ (62\% yield) as colorless oil. ${ }^{1} \mathrm{H}$ NMR analysis revealed that the $\mathrm{dr}$ value was >20:1. HPLC analysis: [Chiralcel AD-H, 3\% ${ }^{i} \mathrm{PrOH} / \mathrm{hexane}, 1.0 \mathrm{~mL} / \mathrm{min}, 230 \mathrm{~nm}$; major diastereomer: $\mathrm{t}_{\mathrm{r}}$ (major) $=34.78 \mathrm{~min}, \mathrm{t}_{\mathrm{r}}$ $($ minor $)=32.09 \mathrm{~min}$ ] gave the isomeric composition of major diastereomer: $94 \%$ ee; $[\alpha]^{25} \mathrm{D}=+1.4\left(\mathrm{c}=0.50, \mathrm{CH}_{2} \mathrm{Cl}_{2}\right) .{ }^{1} \mathrm{H}$ NMR $\left(400 \mathrm{MHz}, \mathrm{CDCl}_{3}\right): \delta 7.78-7.76(\mathrm{~m}$, 1H), 7.70-7.67 (m, 2H), 7.46-7.44 (m, 2H), 7.41-7.40 (m, 1H), 7.38-7.36 (m, 2H), 7.28-7.25 (m, 2H), 7.05-7.02 (m, 1H), 5.33-5.26 (m, 1H), 5.06-4.90 (m, 3H), $4.84(\mathrm{ABd}, J=12.8,4.0 \mathrm{~Hz}, 1 \mathrm{H}), 4.18(\mathrm{dd}$, $J=11.6,4.0 \mathrm{~Hz}, 1 \mathrm{H}), 2.74-2.72(\mathrm{~m}, 2 \mathrm{H}) ;{ }^{13} \mathrm{C} \mathrm{NMR}\left(100 \mathrm{MHz}, \mathrm{CDCl}_{3}\right): \delta 206.5,136.9,136,4,133.1$, $132.9,131.2$, 130.4, 129.3, 129.1, 128.1, 128.0, 127.6, 126.7, 126.5, 126.4, 126.3, 124.9, 123.4, 120.4, 75.7, 65.3, 52.3, 41.2; IR (ATR): 1703, 1557, 1449, 1375, 1078, 930, 903, 820, $752 \mathrm{~cm}^{-1}$; HRMS (ESI): Exact mass calcd for $\mathrm{C}_{23} \mathrm{H}_{19} \mathrm{NNaO}_{3} \mathrm{~S}[\mathrm{M}+\mathrm{Na}]^{+}$: 412.0978, Found: 412.0976. 


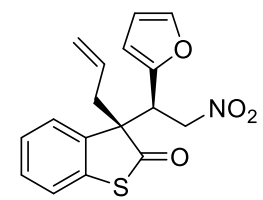

41

Product 41 was obtained in $53.5 \mathrm{mg}$ (65\% yield) as colorless oil. ${ }^{1} \mathrm{H}$ NMR analysis revealed that the dr value was 12:1. HPLC analysis: [Chiralcel IC, 5\% ${ }^{i} \mathrm{PrOH} / \mathrm{hexane}$, $1.0 \mathrm{~mL} / \mathrm{min}, 230 \mathrm{~nm}$; major diastereomer: $\mathrm{t}_{\mathrm{r}}($ major $)=9.63 \mathrm{~min}, \mathrm{t}_{\mathrm{r}}($ minor $)=10.84$ min] gave the isomeric composition of major diastereomer: $90 \%$ ee; $[\alpha]^{25} \mathrm{D}=+6.2(\mathrm{c}$ $\left.=0.50, \mathrm{CHCl}_{3}\right) .{ }^{1} \mathrm{H}$ NMR $\left(400 \mathrm{MHz}, \mathrm{CDCl}_{3}\right): \delta$ 7.36-7.26 (m, 4H), 7.06-7.03 (m, 1H), 6.33-6.32 (m, $1 \mathrm{H}), 6.18(\mathrm{dd}, J=3.2,0.8 \mathrm{~Hz}, 1 \mathrm{H}), 5.28-5.23(\mathrm{~m}, 1 \mathrm{H}), 4.98-4.83(\mathrm{~m}, 3 \mathrm{H}), 4.58(\mathrm{ABd}, J=13.2,3.6 \mathrm{~Hz}$, $1 \mathrm{H}), 4.11(\mathrm{dd}, J=11.6,3.6 \mathrm{~Hz}, 1 \mathrm{H}), 2.76-2.67(\mathrm{~m}, 2 \mathrm{H}) ;{ }^{13} \mathrm{C} \mathrm{NMR}\left(100 \mathrm{MHz}, \mathrm{CDCl}_{3}\right): \delta 205.6,148.1$, $142.8,136.9,135.5,130.3,129.3,126.7,124.9,123.2,120.4,110.7,110.5,73.6,64.4,45.5,40.5 ;$ IR (ATR): 1705, 1557, 1447, 1375, 1339, 1103, 926, 814, $750 \mathrm{~cm}^{-1}$; HRMS (ESI): Exact mass calcd for $\mathrm{C}_{17} \mathrm{H}_{15} \mathrm{NNaO}_{4} \mathrm{~S}[\mathrm{M}+\mathrm{Na}]^{+}:$352.0614, Found: 352.0609 .

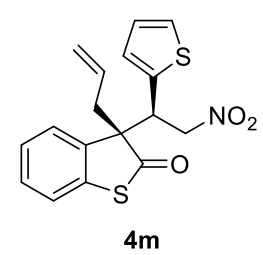

Product $4 \mathrm{~m}$ was obtained in $57.8 \mathrm{mg}$ (67\% yield) as colorless oil. ${ }^{1} \mathrm{H}$ NMR analysis revealed that the $\mathrm{dr}$ value was >20:1. HPLC analysis: [Chiralcel AD-H, 3\% ${ }^{i} \mathrm{PrOH} /$ hexane, $1.0 \mathrm{~mL} / \mathrm{min}, 230 \mathrm{~nm}$; major diastereomer: $\mathrm{t}_{\mathrm{r}}$ (major) $=20.39 \mathrm{~min}, \mathrm{t}_{\mathrm{r}}$ $($ minor $)=14.33 \mathrm{~min}]$ gave the isomeric composition of major diastereomer: $88 \%$ ee; $[\alpha]^{25} \mathrm{D}=+4.4\left(\mathrm{c}=0.50, \mathrm{CH}_{2} \mathrm{Cl}_{2}\right) .{ }^{1} \mathrm{H}$ NMR $\left(400 \mathrm{MHz}, \mathrm{CDCl}_{3}\right): \delta$ 7.39-7.33 (m, 3H), 7.22-7.18 (m, 2H), $6.88(\mathrm{dd}, J=5.2,3,6 \mathrm{~Hz}, 1 \mathrm{H}), 6.77(\mathrm{dd}, J=3.6,1.2 \mathrm{~Hz}, 1 \mathrm{H}), 5.33-5.27(\mathrm{~m}, 1 \mathrm{H}), 5.00-4.93(\mathrm{~m}$, $2 \mathrm{H}), 4.81-4.77(\mathrm{~m}, 2 \mathrm{H}), 4.35(\mathrm{dd}, J=10.8,4.4 \mathrm{~Hz}, 1 \mathrm{H}), 2.78-2.70(\mathrm{~m}, 2 \mathrm{H}) ;{ }^{13} \mathrm{C} \mathrm{NMR}(100 \mathrm{MHz}$, $\left.\mathrm{CDCl}_{3}\right): \delta 206.3,136.7,136.6,135.7,130.2,129.5,128.7,126.7,126.6,126.3,124.9,123.4,120.5$, 76.7, 65.0, 47.6, 41.3; IR (ATR): 1703, 1557, 1447, 1377, 1339, 1101, 928, 853, $752 \mathrm{~cm}^{-1}$; HRMS (ESI): Exact mass calcd for $\mathrm{C}_{17} \mathrm{H}_{15} \mathrm{NNaO}_{3} \mathrm{~S}_{2}[\mathrm{M}+\mathrm{Na}]^{+}:$368.0386, Found: 368.0383.

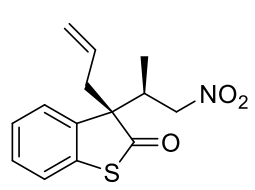

$4 n$

Product 4n was obtained in $42.9 \mathrm{mg}$ (62\% yield) as colorless oil. ${ }^{1} \mathrm{H}$ NMR analysis revealed that the $\mathrm{dr}$ value was 5:1. HPLC analysis: [Chiralcel AD-H, 3\% ${ }^{i} \mathrm{PrOH} /$ hexane, $1.0 \mathrm{~mL} / \mathrm{min}, 230 \mathrm{~nm}$; major diastereomer: $\mathrm{t}_{\mathrm{r}}$ (major) $=8.65 \mathrm{~min}, \mathrm{t}_{\mathrm{r}}$ $($ minor $)=8.12 \mathrm{~min}]$ gave the isomeric composition of major diastereomer: $80 \%$ ee; $[\alpha]^{25}{ }_{\mathrm{D}}=+3.5\left(\mathrm{c}=0.50, \mathrm{CH}_{2} \mathrm{Cl}_{2}\right) .{ }^{1} \mathrm{H}$ NMR $\left(400 \mathrm{MHz}, \mathrm{CDCl}_{3}\right): \delta$ 7.38-7.24 (m, 4H), 5.35-5.31 (m, $1 \mathrm{H}), 5.03-4.95(\mathrm{~m}, 2 \mathrm{H}), 4.22-4.11(\mathrm{~m}, 2 \mathrm{H}), 2.95-2.92(\mathrm{~m}, 1 \mathrm{H}), 2.86(\mathrm{ABd}, J=13.2,7.6 \mathrm{~Hz}, 1 \mathrm{H})$, 2.62-2.57 (m, 2H), $1.22(\mathrm{~d}, J=6.8 \mathrm{~Hz}, 3 \mathrm{H}) ;{ }^{13} \mathrm{C} \mathrm{NMR}\left(125 \mathrm{MHz}, \mathrm{CDCl}_{3}\right): \delta 206.6,137.7,135.6$, 130.4, 129.1, 127.0, 124.5, 123.3, 120.4, 63.9, 41.6, 41.4, 12.1; IR (ATR): 1703, 1555, 1449, 1387, 1377, 1229, 999, 768, $750 \mathrm{~cm}^{-1}$; HRMS (ESI): Exact mass calcd for $\mathrm{C}_{14} \mathrm{H}_{15} \mathrm{NNaO}_{3} \mathrm{~S}[\mathrm{M}+\mathrm{Na}]^{+}$: 300.0665, Found: 300.0658. 


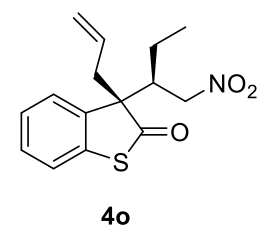

Product 40 was obtained in $45.8 \mathrm{mg}$ (63\% yield) as colorless oil. ${ }^{1} \mathrm{H}$ NMR analysis revealed that the $\mathrm{dr}$ value was 6:1. HPLC analysis: [Chiralcel AD-H, 3\% ${ }^{i} \mathrm{PrOH} / \mathrm{hexane}, 1.0 \mathrm{~mL} / \mathrm{min}, 230 \mathrm{~nm}$; major diastereomer: $\mathrm{t}_{\mathrm{r}}$ (major) $=9.39 \mathrm{~min}, \mathrm{t}_{\mathrm{r}}$ $($ minor $)=7.88 \mathrm{~min}]$ gave the isomeric composition of major diastereomer: $87 \%$ ee; $[\alpha]^{25} \mathrm{D}=+4.5\left(\mathrm{c}=0.50, \mathrm{CH}_{2} \mathrm{Cl}_{2}\right) .{ }^{1} \mathrm{H}$ NMR $\left(400 \mathrm{MHz}, \mathrm{CDCl}_{3}\right): \delta$ 7.39-7.22 (m, 4H), 5.37-5.28 (m, $1 \mathrm{H}), 5.02-4.94(\mathrm{~m}, 2 \mathrm{H}), 4.28-4.26(\mathrm{~m}, 2 \mathrm{H}), 2.87-2.82(\mathrm{~m}, 2 \mathrm{H}), 2.64-2.58(\mathrm{~m}, 1 \mathrm{H}), 1.96-1.91(\mathrm{~m}, 1 \mathrm{H})$, 1.45-1.41 (m, 1H), $0.94(\mathrm{t}, J=7.2 \mathrm{~Hz}, 3 \mathrm{H}) ;{ }^{13} \mathrm{C} \mathrm{NMR}\left(125 \mathrm{MHz}, \mathrm{CDCl}_{3}\right): \delta 207.1,137.8,135.6$, 130.4, 129.1, 126.8, 124.7, 123.3, 120.3, 76.8, 65.0, 47.5, 41.7, 21.5, 12.4; IR (ATR): 1705, 1555, 1468, 1379, 1225, 926, 914, $748 \mathrm{~cm}^{-1}$; HRMS (ESI): Exact mass calcd for $\mathrm{C}_{15} \mathrm{H}_{17} \mathrm{NNaO}_{3} \mathrm{~S}[\mathrm{M}+\mathrm{Na}]^{+}$: 314.0821, Found: 314.0814.

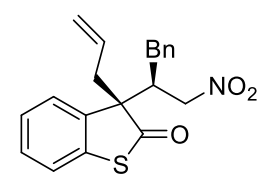

$4 p$

Product $4 \mathbf{p}$ was obtained in $53.0 \mathrm{mg}$ (60\% yield) as colorless oil. ${ }^{1} \mathrm{H}$ NMR analysis revealed that the $\mathrm{dr}$ value was 6:1. HPLC analysis: [Chiralcel AD-H, 3\% ${ }^{i} \mathrm{PrOH} / \mathrm{hexane}, 1.0 \mathrm{~mL} / \mathrm{min}, 230 \mathrm{~nm}$; major diastereomer: $\mathrm{t}_{\mathrm{r}}$ (major) $=12.57 \mathrm{~min}, \mathrm{t}_{\mathrm{r}}$ $($ minor $)=11.47 \mathrm{~min}]$ gave the isomeric composition of major diastereomer: $85 \%$ ee; $[\alpha]^{25} \mathrm{D}=+5.7\left(\mathrm{c}=0.50, \mathrm{CH}_{2} \mathrm{Cl}_{2}\right) .{ }^{1} \mathrm{H}$ NMR $\left(400 \mathrm{MHz}, \mathrm{CDCl}_{3}\right): \delta$ 7.40-7.33 $(\mathrm{m}, 2 \mathrm{H}), 7.32-7.26(\mathrm{~m}$, 4H), 7.25-7.22 (m, 1H), 7.20-7.17 (m, 2H), 5.35-5.30 (m, 1H), 5.03-4.96 (m, 2H), 4.28-4.18 (m, 2H), 3,38-3.35 (m, 1H), 3.29 (dd, $J=14.4,4.4 \mathrm{~Hz}, 1 \mathrm{H}), 2.89$ (ABd, $J=13.2,7.6 \mathrm{~Hz}, 1 \mathrm{H}), 2.64-2.52(\mathrm{~m}$, 2H); ${ }^{13} \mathrm{C}$ NMR (125 MHz, $\mathrm{CDCl}_{3}$ ): $\delta$ 207.3, 137.6, 137.3, 135.6, 130.3, 129.2, 129.1, 128.8, 127.1, 126.9, 125.0, 123.4, 120.5, 75.3, 65.0, 47.1, 41.8, 33.8; IR (ATR): 1703, 1555, 1449, 1379, 1140, 1078, 926, 748, $702 \mathrm{~cm}^{-1}$; HRMS (ESI): Exact mass calcd for $\mathrm{C}_{20} \mathrm{H}_{19} \mathrm{NNaO}_{3} \mathrm{~S}[\mathrm{M}+\mathrm{Na}]^{+}: 376.0978$, Found: 376.0973 .

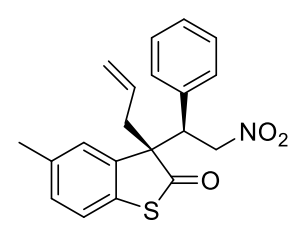

$4 q$

Product 4q was obtained in $51.2 \mathrm{mg}$ (58\% yield) as colorless oil. ${ }^{1} \mathrm{H}$ NMR analysis revealed that the dr value was 19:1. HPLC analysis: [Chiralcel AD-H, 2\% ${ }^{i} \mathrm{PrOH} / \mathrm{hexane}, 1.0 \mathrm{~mL} / \mathrm{min}, 230 \mathrm{~nm}$; major diastereomer: $\mathrm{t}_{\mathrm{r}}$ (major) $=13.72 \mathrm{~min}$, $\mathrm{t}_{\mathrm{r}}$ $($ minor $)=11.82 \mathrm{~min}]$ gave the isomeric composition of major diastereomer: $89 \%$ ee; $[\alpha]^{25}{ }_{\mathrm{D}}=-9.6\left(\mathrm{c}=0.50, \mathrm{CH}_{2} \mathrm{Cl}_{2}\right) .{ }^{1} \mathrm{H}$ NMR $\left(400 \mathrm{MHz}, \mathrm{CDCl}_{3}\right): \delta$ 7.28-7.21 (m, $3 \mathrm{H}), 7.18-7.17(\mathrm{~m}, 2 \mathrm{H}), 6.98-6.95(\mathrm{~m}, 3 \mathrm{H}), 5.30-5.22(\mathrm{~m}, 1 \mathrm{H}), 4.98-4.89(\mathrm{~m}, 3 \mathrm{H}), 4.75(\mathrm{ABd}, J=12.8$, $4.0 \mathrm{~Hz}, 1 \mathrm{H}), 3.97(\mathrm{dd}, J=11.6,4.0 \mathrm{~Hz}, 1 \mathrm{H}), 2.66-2.64(\mathrm{~m}, 2 \mathrm{H}), 2.41(\mathrm{~s}, 3 \mathrm{H}) ;{ }^{13} \mathrm{C}$ NMR $(100 \mathrm{MHz}$, $\left.\mathrm{CDCl}_{3}\right): \delta 206.8,136.8,136.6,133.7,132.7,130.5,130.1,129.4,128.5,128.3,125.5,123.0,120.2$, 75.6, 65.0, 52.2, 41.1, 21.5; IR (ATR): 1701, 1557, 1456, 1377, 1339, 1150, 928, 814, $739 \mathrm{~cm}^{-1}$; 
HRMS (ESI): Exact mass calcd for $\mathrm{C}_{20} \mathrm{H}_{19} \mathrm{NNaO}_{3} \mathrm{~S}[\mathrm{M}+\mathrm{Na}]^{+}$: 376.0978, Found: 376.0973 .

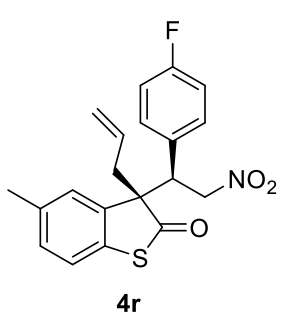

Product $4 \mathbf{r}$ was obtained in $57.5 \mathrm{mg}$ (62\% yield) as colorless oil. ${ }^{1} \mathrm{H}$ NMR analysis revealed that the dr value was >20:1. HPLC analysis: [Chiralcel AD-H, 3\% ${ }^{i} \mathrm{PrOH} / \mathrm{h}$ exane, $1.0 \mathrm{~mL} / \mathrm{min}, 230 \mathrm{~nm}$; major diastereomer: $\mathrm{t}_{\mathrm{r}}$ (major) $=14.99 \mathrm{~min}, \mathrm{t}_{\mathrm{r}}$ $($ minor $)=12.95 \mathrm{~min}]$ gave the isomeric composition of major diastereomer: $91 \%$ ee; $[\alpha]^{25} \mathrm{D}=-10.9\left(\mathrm{c}=0.50, \mathrm{CH}_{2} \mathrm{Cl}_{2}\right) .{ }^{1} \mathrm{H}$ NMR $\left(400 \mathrm{MHz}, \mathrm{CDCl}_{3}\right): \delta 7.19(\mathrm{~d}, J=$ $1.2 \mathrm{~Hz}, 2 \mathrm{H}), 7.02-7.01(\mathrm{~m}, 1 \mathrm{H}), 6.92-6.88(\mathrm{~m}, 4 \mathrm{H}), 5.31-5.24(\mathrm{~m}, 1 \mathrm{H}), 4.99-4.83(\mathrm{~m}, 3 \mathrm{H}), 4.73(\mathrm{ABd}$, $J=12.8,4.4 \mathrm{~Hz}, 1 \mathrm{H}), 3.98(\mathrm{dd}, J=12.0,4.4 \mathrm{~Hz}, 1 \mathrm{H}), 2.65-2.63(\mathrm{~m}, 2 \mathrm{H}), 2.44(\mathrm{~s}, 3 \mathrm{H}) ;{ }^{13} \mathrm{C}$ NMR $(100$ $\left.\mathrm{MHz}, \mathrm{CDCl}_{3}\right): \delta 206.8,163.7\left(\mathrm{~d}, J_{\mathrm{F}-\mathrm{C}}=247.0 \mathrm{~Hz}\right), 136.8,136.6,132.8,131.0\left(\mathrm{~d}, J_{\mathrm{F}-\mathrm{C}}=8.0 \mathrm{~Hz}\right), 130.3$ $\left(\mathrm{d}, J_{\mathrm{F}-\mathrm{C}}=3.0 \mathrm{~Hz}\right), 129.4\left(\mathrm{~d}, J_{\mathrm{F}-\mathrm{C}}=3.0 \mathrm{~Hz}\right), 125.3,123.1,120.3,115.4\left(\mathrm{~d}, J_{\mathrm{F}-\mathrm{C}}=21.0 \mathrm{~Hz}\right), 75.7,65.0$, 51.5, 41.2, 21.5; ${ }^{19} \mathrm{~F}$ NMR (376 MHz, $\mathrm{CDCl}_{3}$ ): $\delta$-113.16; IR (ATR): 1703, 1557, 1472, 1377, 1229 , 1105, 926, 841, $735 \mathrm{~cm}^{-1}$; HRMS (ESI): Exact mass calcd for $\mathrm{C}_{20} \mathrm{H}_{18} \mathrm{FNNaO}_{3} \mathrm{~S}[\mathrm{M}+\mathrm{Na}]^{+}: 394.0884$, Found: 394.0881.

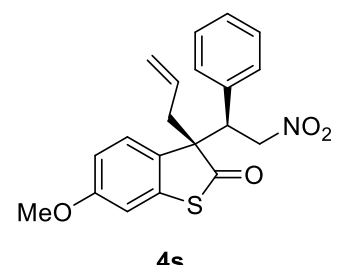

Product $4 \mathrm{~s}$ was obtained in $53.5 \mathrm{mg}$ (58\% yield) as colorless oil. ${ }^{1} \mathrm{H}$ NMR analysis revealed that the dr value was >20:1. HPLC analysis: [Chiralcel IC, 5\% ${ }^{i} \mathrm{PrOH} / \mathrm{hexane}, 1.0 \mathrm{~mL} / \mathrm{min}, 230 \mathrm{~nm}$; major diastereomer: $\mathrm{t}_{\mathrm{r}}$ (major) $=15.57 \mathrm{~min}$, $\left.t_{\mathrm{r}}(\operatorname{minor})=24.08 \mathrm{~min}\right]$ gave the isomeric composition of major diastereomer: $85 \%$ ee; $[\alpha]^{25}{ }_{\mathrm{D}}=+2.5\left(\mathrm{c}=0.50, \mathrm{CH}_{2} \mathrm{Cl}_{2}\right) .{ }^{1} \mathrm{H} \mathrm{NMR}\left(400 \mathrm{MHz}, \mathrm{CDCl}_{3}\right): \delta$ 7.26-7.19 (m, 3H), $7.07(\mathrm{~d}$, $J=8.4 \mathrm{~Hz}, 1 \mathrm{H}), 6.94-6.82(\mathrm{~m}, 4 \mathrm{H}), 5.32-5.25(\mathrm{~m}, 1 \mathrm{H}), 4.98-4.87$ (m, 3H), 4.79 (ABd, $J=12.8,4.4$ $\mathrm{Hz}, 1 \mathrm{H}), 3.96(\mathrm{dd}, J=11.2,4.4 \mathrm{~Hz}, 1 \mathrm{H}), 3.84(\mathrm{~s}, 3 \mathrm{H}), 2.67-2.64(\mathrm{~m}, 2 \mathrm{H}) ;{ }^{13} \mathrm{C}$ NMR $(125 \mathrm{MHz}$, $\left.\mathrm{CDCl}_{3}\right): \delta 206.8,160.1,137.4,133.8,130.6,129.4,128.5,128.4,128.3,125.6,120.2,112.7,108.8$, 75.7, 64.6, 55.6, 52.2, 41.2; IR (ATR): 1705, 1557, 1456, 1377, 1312, 1088, 928, 914, $737 \mathrm{~cm}^{-1}$; HRMS (ESI): Exact mass calcd for $\mathrm{C}_{20} \mathrm{H}_{19} \mathrm{NNaO}_{4} \mathrm{~S}[\mathrm{M}+\mathrm{Na}]^{+}$: 392.0927, Found: 392.0927.

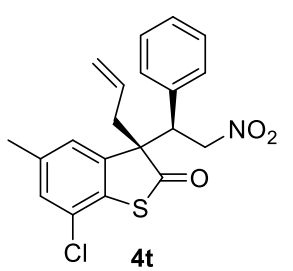

Product $4 \mathbf{t}$ was obtained in $54.2 \mathrm{mg}$ ( $56 \%$ yield) as colorless oil. ${ }^{1} \mathrm{H}$ NMR analysis revealed that the dr value was 5:1. HPLC analysis: [Chiralcel AD-H, 2\% ${ }^{i} \mathrm{PrOH} /$ hexane, $1.0 \mathrm{~mL} / \mathrm{min}, 230 \mathrm{~nm}$; major diastereomer: $\mathrm{t}_{\mathrm{r}}$ (major) $=10.92 \mathrm{~min}, \mathrm{t}_{\mathrm{r}}$ $($ minor $)=9.59 \mathrm{~min}]$ gave the isomeric composition of major diastereomer: $81 \%$ ee; $[\alpha]^{25} \mathrm{D}=+1.5\left(\mathrm{c}=0.50, \mathrm{CH}_{2} \mathrm{Cl}_{2}\right) .{ }^{1} \mathrm{H}$ NMR $\left(400 \mathrm{MHz}, \mathrm{CDCl}_{3}\right): \delta$ 7.29-7.21 (m, 4H), 6.98-6.96 (m, 2H), 6.83-6.82 (m, 1H), 5.27-5.22 (m, 1H), 4.98-4.88 (m, 3H), $4.74(\mathrm{ABd}, J=13.2,4.4 \mathrm{~Hz}, 1 \mathrm{H}), 3.96$ 
$(\mathrm{dd}, J=11.6,4.4 \mathrm{~Hz}, 1 \mathrm{H}), 2.65-2.62(\mathrm{~m}, 2 \mathrm{H}), 2.40(\mathrm{~s}, 3 \mathrm{H}) ;{ }^{13} \mathrm{C} \mathrm{NMR}\left(100 \mathrm{MHz}, \mathrm{CDCl}_{3}\right): \delta 205.3$, 138.5, 138.0, 133.3, 132.8, 130.1, 130.0, 129.4, 128.7, 128.4, 128.3, 123.7, 120.7, 75.4, 66.7, 52.1, 41.0, 21.3; IR (ATR): 1709, 1557, 1441, 1377, 1038, 908, 856, 733, $700 \mathrm{~cm}^{-1}$; HRMS (ESI): Exact mass calcd for $\mathrm{C}_{20} \mathrm{H}_{18} \mathrm{ClNNaO}_{3} \mathrm{~S}[\mathrm{M}+\mathrm{Na}]^{+}$: 410.0588, Found: 410.0590.

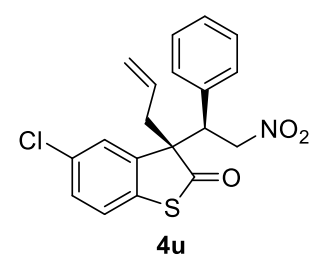

The reaction was conducted at $-90{ }^{\circ} \mathrm{C}$. Product $4 \mathbf{u}$ was obtained in $62.5 \mathrm{mg}(67 \%$ yield) as colorless oil. ${ }^{1} \mathrm{H}$ NMR analysis revealed that the dr value was 6:1. HPLC analysis: [Chiralcel AD-H, 3\% ${ }^{i} \mathrm{PrOH} /$ hexane, $1.0 \mathrm{~mL} / \mathrm{min}, 230 \mathrm{~nm}$; major diastereomer: $\mathrm{t}_{\mathrm{r}}($ major $)=13.92 \mathrm{~min}, \mathrm{t}_{\mathrm{r}}($ minor $\left.)=12.17 \mathrm{~min}\right]$ gave the isomeric composition of major diastereomer: $70 \%$ ee; $[\alpha]^{25} \mathrm{D}=-10.2\left(\mathrm{c}=0.50, \mathrm{CHCl}_{3}\right) .{ }^{1} \mathrm{H} \mathrm{NMR}(400 \mathrm{MHz}$, $\left.\mathrm{CD}_{2} \mathrm{Cl}_{2}\right): \delta 7.31-7.28(\mathrm{~m}, 1 \mathrm{H}), 7.31-7.07(\mathrm{~m}, 4 \mathrm{H}), 7.07(\mathrm{~d}, J=2.0 \mathrm{~Hz}, 1 \mathrm{H}), 6.88-6.86(\mathrm{~m}, 2 \mathrm{H})$, 5.23-5.20 (m, 1H), 4.92-4.84 (m, 3H), $4.75(\mathrm{ABd}, J=13.2,4.4 \mathrm{~Hz}, 1 \mathrm{H}), 3.89(\mathrm{dd}, J=12.0,4.4 \mathrm{~Hz}$, 1H), 2.61 (dt, $J=7.2,1.2 \mathrm{~Hz}, 2 \mathrm{H}) ;{ }^{13} \mathrm{C} \mathrm{NMR}\left(125 \mathrm{MHz}, \mathrm{CD}_{2} \mathrm{Cl}_{2}\right)$ : $\delta$ 205.5, 138.6, 134.6, 133.5, 132.6, 130.2, 129.5, 129.4, 128.6, 128.4, 125.3, 124.3, 120.4, 75.3, 51.8, 40.8; IR (ATR): 1707, 1557, 1456, 1377, 1094, 930, 818, 754, $702 \mathrm{~cm}^{-1}$; HRMS (ESI): Exact mass calcd for $\mathrm{C}_{19} \mathrm{H}_{16} \mathrm{ClNNaO}_{3} \mathrm{~S}[\mathrm{M}+\mathrm{Na}]^{+}$: 396.0432, Found: 396.0434.

\section{(b) General procedure for $1.1 \mathrm{mmol}$ scale reaction}

Under an atmosphere of nitrogen, to a Schlenk tube were added ${ }^{t} \mathrm{Bu}_{2} \mathrm{CyPAuCl}(10.1 \mathrm{mg}, 0.022$ mmol), AgOTf $(8.8 \mathrm{mg}, 0.033 \mathrm{mmol})$, and anhydrous $\mathrm{CH}_{2} \mathrm{Cl}_{2}(11 \mathrm{~mL})$. The resulting mixture was stirred at room temperature for 15-20 minutes, and followed by the addition of allyltrimethylsilane $2 \mathbf{a}$ $(0.88 \mathrm{~mL}, 5.5 \mathrm{mmol})$ and diazothiooxindole $1 \mathbf{a}(1.1 \mathrm{mmol})$. The reaction was kept stirring at $25{ }^{\circ} \mathrm{C}$ for about $22 \mathrm{~h}$ till full conversion of diazothiooxindole 1a by TLC analysis. Then $\mathrm{CH}_{2} \mathrm{Cl}_{2}$ was removed under reduced pressure, and the residue was directly subjected to a short column chromatography to remove ${ }^{t} \mathrm{Bu}_{2} \mathrm{CyPAuOTf}$ using an eluent of $\operatorname{EtOAc} / \mathrm{PE}(1: 15, \mathrm{v} / \mathrm{v})$. After concentration, toluene $(44 \mathrm{~mL})$, powdered MS $3 \AA$ (1.1 g) and chiral catalyst C1 (50.2 mg, $0.11 \mathrm{mmol})$ were added successively. After cooling down to $-78{ }^{\circ} \mathrm{C}$ for 30 minutes, the nitroolefins $10(192.5 \mathrm{mg}, 1.1 \mathrm{mmol})$ was added. The resulting mixture was stirred till full conversion of $\mathbf{6 a}$ by TLC analysis. Then the solvent was carefully removed under reduced pressure. To determine the diastereoselectivity of product, the residue was first dissolved in $\mathrm{CDCl}_{3}$, and some samples were taken for NMR analysis. Then the sample for analysis and the rest of the product were recombined for column chromatographic purification using EtOAc/PE (from 1:50 to 1:10, v/v) as the eluent to afford product 4a was obtained in $228.9 \mathrm{mg}(57 \%$ yield), $92 \%$ ee, $9: 1 \mathrm{dr}$. 
(c) Tandem reaction based on diazooxindoles 8

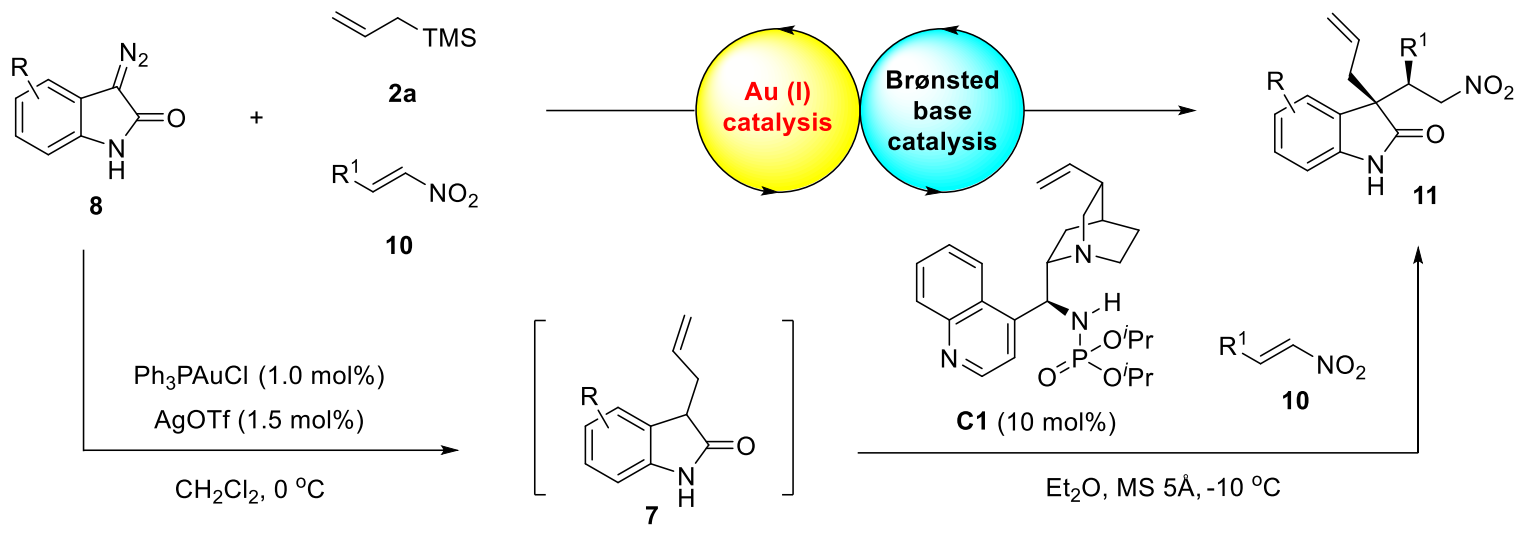

Under an atmosphere of nitrogen, to a Schlenk tube were added $\mathrm{Ph}_{3} \mathrm{PAuCl}(1.2 \mathrm{mg}, 0.0025 \mathrm{mmol})$ and $\mathrm{AgOTf}(1.0 \mathrm{mg}, 0.0038 \mathrm{mmol})$, followed by anhydrous $\mathrm{CH}_{2} \mathrm{Cl}_{2}(4.0 \mathrm{~mL})$. The resulting mixture was stirred at room temperature for $15-20$ minutes, and then cooled down to $0{ }^{\circ} \mathrm{C}$, followed by the addition of allyltrimethylsilane $\mathbf{2 a}(0.2 \mathrm{~mL}, 1.25 \mathrm{mmol})$ and diazooxindole $8(0.25 \mathrm{mmol})$. The reaction was kept stirring at $0{ }^{\circ} \mathrm{C}$ for about $1-24 \mathrm{~h}$ till full conversion of diazooxindole 8 by TLC analysis. After the anhydrous $\mathrm{CH}_{2} \mathrm{Cl}_{2}$ and excess silane $\mathbf{2 a}$ were removed under reduced pressure, ordinary $\mathrm{CH}_{2} \mathrm{Cl}_{2}$ (not anhydrous, $4 \mathrm{~mL}$ ) was added. The solution was kept stirring at $25{ }^{\circ} \mathrm{C}$ for about 0.5-24 $\mathrm{h}$ till the ring-opening process finished by TLC analysis. Then $\mathrm{CH}_{2} \mathrm{Cl}_{2}$ was removed under reduced pressure, $\mathrm{Et}_{2} \mathrm{O}(4.0 \mathrm{~mL})$, powdered MS $5 \AA$ (125 mg) and chiral catalyst $\mathbf{C 1}$ (11.4 mg, 0.025 mmol) were added successively. After cooling down to $-10{ }^{\circ} \mathrm{C}$ for 30 minutes, the nitroenynes 10 $(0.25 \mathrm{mmol})$ was added. The resulting mixture was stirred till full conversion of $\mathbf{7}$ by TLC analysis. and then the solvent was carefully removed under reduced pressure. To determine the diastereoselectivity of product, the residue was first dissolved in $\mathrm{CDCl}_{3}$, and some samples were taken for NMR analysis. Then the sample for analysis and the rest of the product were recombined for column chromatographic purification using petroleum ether/ $\mathrm{CH}_{2} \mathrm{Cl}_{2} /$ acetone (from 50:100:0 to $0: 100: 1, \mathrm{v}: \mathrm{v})$ as the eluent.

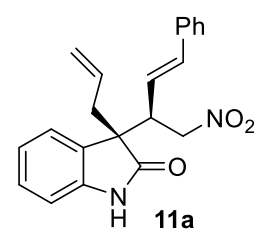

Product 11a was obtained in $51.3 \mathrm{mg}$ (59\% yield) as yellowish oil. ${ }^{1} \mathrm{H}$ NMR analysis revealed that the dr value was >20:1. HPLC analysis: [Chiralcel AD-H, 15\% ${ }^{i} \mathrm{PrOH} / \mathrm{h}$ exane, $1.0 \mathrm{~mL} / \mathrm{min}, 254 \mathrm{~nm}$; major diastereomer: $\mathrm{t}_{\mathrm{r}}$ (major) $=20.13 \mathrm{~min}, \mathrm{t}_{\mathrm{r}}$ $(\operatorname{minor})=23.38 \mathrm{~min}]$ gave the isomeric composition of major diastereomer: $95 \%$ ee; $[\alpha]^{25} \mathrm{D}=+17.2\left(\mathrm{c}=1.00, \mathrm{CHCl}_{3}\right) .{ }^{1} \mathrm{H}$ NMR $\left(400 \mathrm{MHz}, \mathrm{CDCl}_{3}\right): \delta 8.64(\mathrm{~s}, 1 \mathrm{H}), 7.37-7.35(\mathrm{~m}, 2 \mathrm{H})$, 
7.32-7.14 (m, 5H), $7.13(\mathrm{td}, J=7.6,1.2 \mathrm{~Hz}, 1 \mathrm{H}), 6.95(\mathrm{~d}, J=7.6 \mathrm{~Hz}, 1 \mathrm{H}), 6.56(\mathrm{~d}, J=15.6 \mathrm{~Hz}, 1 \mathrm{H})$, 6.44-6.38 (m, 1H), 5.40-5.34 (m, 1H), 5.01-4.91 (m, 2H), $4.34(\mathrm{t}, J=11.2 \mathrm{~Hz}, 1 \mathrm{H}), 4.18(\mathrm{ABd}, J=$ 11.6, 3.2 Hz, 1H), $3.52(\mathrm{td}, J=10.0,3.6 \mathrm{~Hz}, 1 \mathrm{H}), 2.69,2.60(\mathrm{ABd}, J=13.6,8.0 \mathrm{~Hz}, 2 \mathrm{H}) ;{ }^{13} \mathrm{C} \mathrm{NMR}$ $\left(125 \mathrm{MHz}, \mathrm{CDCl}_{3}\right): \delta 179.2,140.8,136.4,136.1,130.8,129.6,129.0,128.6,128.1,126.8,123.7$, 123.2, 122.5, 120.0, 110.2, 76.3, 54.0, 48.9, 40.2; IR (ATR): 1711, 1558, 1472, 1379, 1319, 1248, 910, 756, $692 \mathrm{~cm}^{-1}$; HRMS (ESI): Exact mass calcd for $\mathrm{C}_{21} \mathrm{H}_{20} \mathrm{~N}_{2} \mathrm{NaO}_{3}[\mathrm{M}+\mathrm{Na}]^{+}$: 371.1366 , Found: 371.1365 .

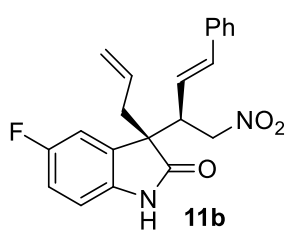

Product 11b was obtained in $75.4 \mathrm{mg}$ (84\% yield) as yellowish oil. ${ }^{1} \mathrm{H} \mathrm{NMR}$ analysis revealed that the dr value was 20:1. HPLC analysis: [Chiralcel AD-H, $15 \%{ }^{i} \mathrm{PrOH} /$ hexane, $1.0 \mathrm{~mL} / \mathrm{min}, 254 \mathrm{~nm}$; major diastereomer: $\mathrm{t}_{\mathrm{r}}$ (major) $=10.98$ $\min , \mathrm{t}_{\mathrm{r}}($ minor $\left.)=13.13 \mathrm{~min}\right]$ gave the isomeric composition of major diastereomer: $97 \%$ ee; $[\alpha]^{25} \mathrm{D}=+8.9\left(\mathrm{c}=1.00, \mathrm{CHCl}_{3}\right) .{ }^{1} \mathrm{H} \mathrm{NMR}\left(400 \mathrm{MHz}, \mathrm{CDCl}_{3}\right): \delta 8.38(\mathrm{~s}, 1 \mathrm{H}), 7.39-7.26(\mathrm{~m}$, 5H), 7.04-6.99 (m, 2H), 6.90-6.87 (m, 1H), 6.57 (d, J=16.0 Hz, 1H), 6.43-6.37 (m, 1H), 5.46-5.36 (m, $1 \mathrm{H}), 5.41-5.34(\mathrm{~m}, 1 \mathrm{H}), 5.04-4.95(\mathrm{~m}, 2 \mathrm{H}), 4.35(\mathrm{t}, J=11.6 \mathrm{~Hz}, 1 \mathrm{H}), 4.19(\mathrm{ABd}, J=12.0,3.6 \mathrm{~Hz}, 1 \mathrm{H})$, 3.51-3.49 (m, 1H), 2.69, $2.58(\mathrm{ABd}, J=13.6,7.6 \mathrm{~Hz}, 2 \mathrm{H}) ;{ }^{13} \mathrm{C} \mathrm{NMR}\left(125 \mathrm{MHz}, \mathrm{CDCl}_{3}\right): \delta 179.1$, $159.3\left(\mathrm{~d}, J_{\mathrm{C}-\mathrm{F}}=193.0 \mathrm{~Hz}\right), 136.7,136.0,131.3\left(\mathrm{~d}, J_{\mathrm{C}-\mathrm{F}}=7.5 \mathrm{~Hz}\right), 130.4,128.6,128.3,126.8,122.0$, $120.4,115.6\left(\mathrm{~d}, J_{\mathrm{C}-\mathrm{F}}=22.5 \mathrm{~Hz}\right), 111.7\left(\mathrm{~d}, J_{\mathrm{C}-\mathrm{F}}=25.0 \mathrm{~Hz}\right), 110.9\left(\mathrm{~d}, J_{\mathrm{C}-\mathrm{F}}=7.5 \mathrm{~Hz}\right), 76.2,54.6,54.5$, 48.8, 40.1; ${ }^{19} \mathrm{~F}$ NMR (376 MHz, $\mathrm{CDCl}_{3}$ ): $\delta$-118.67; IR (ATR): 1715, 1558, 1487, 1288, 1248, 910, 779, 760, $737 \mathrm{~cm}^{-1}$; HRMS (ESI): Exact mass calcd for $\mathrm{C}_{21} \mathrm{H}_{19} \mathrm{~N}_{2} \mathrm{NaO}_{3} \mathrm{~F}[\mathrm{M}+\mathrm{Na}]^{+}:$382.1272, Found: 389.1269 .

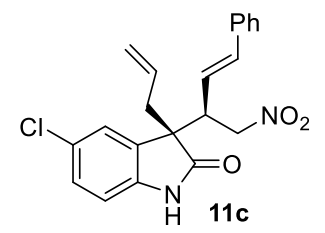

Product 11c was obtained in $82.1 \mathrm{mg}$ (86\% yield) as yellowish oil. ${ }^{1} \mathrm{H}$ NMR analysis revealed that the dr value was 16:1. HPLC analysis: [Chiralcel AD-H, $15 \%{ }^{i} \mathrm{PrOH} /$ hexane, $1.0 \mathrm{~mL} / \mathrm{min}, 254 \mathrm{~nm}$; major diastereomer: $\mathrm{t}_{\mathrm{r}}$ (major) $=10.40$ min, $\left.t_{r}(\operatorname{minor})=12.28 \mathrm{~min}\right]$ gave the isomeric composition of major diastereomer: $97 \%$ ee; $[\alpha]^{25}=+18.7\left(\mathrm{c}=1.00, \mathrm{CHCl}_{3}\right) .{ }^{1} \mathrm{H} \mathrm{NMR}\left(400 \mathrm{MHz}, \mathrm{CDCl}_{3}\right): \delta 8.89(\mathrm{~s}, 1 \mathrm{H}), 7.35-7.29(\mathrm{~m}$, 2H), 7.29-7.26 (m, 5H), $6.90(\mathrm{~d}, J=8.4 \mathrm{~Hz}, 1 \mathrm{H}), 6.57(\mathrm{~d}, J=16.0 \mathrm{~Hz}, 1 \mathrm{H}), 6.39-6.32(\mathrm{~m}, 1 \mathrm{H})$, 5.39-5.33 (m, 1H), 5.03-4.94 (m, 2H), $4.34(\mathrm{t}, J=12.0,3.6 \mathrm{~Hz}, 1 \mathrm{H}), 4.19(\mathrm{ABd}, J=11.6,3.6 \mathrm{~Hz}, 1 \mathrm{H})$, 3.53-3.49 (m, 1H), 2.68, $2.58(\mathrm{ABd}, J=13.6,7.6 \mathrm{~Hz}, 2 \mathrm{H}) ;{ }^{13} \mathrm{C} \mathrm{NMR}\left(100 \mathrm{MHz}, \mathrm{CDCl}_{3}\right): \delta 179.1$, 139.4, 136.8, 135.9, 131.4, 130.3, 129.1, 128.6, 128.3, 126.8, 124.1, 122.0, 120.5, 111.3, 76.1, 54.4, 48.7, 40.1; IR (ATR): 1709, 1557, 1478, 1435, 1377, 1287, 910,775, $737 \mathrm{~cm}^{-1}$; HRMS (ESI): Exact 
mass calcd for $\mathrm{C}_{21} \mathrm{H}_{19} \mathrm{~N}_{2} \mathrm{NaO}_{3} \mathrm{Cl}[\mathrm{M}+\mathrm{Na}]^{+}$: 405.0976, Found: 405.0976.

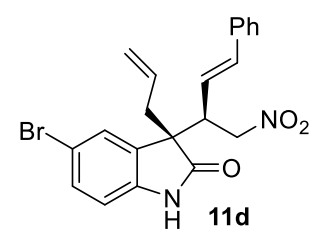

Product 11d was obtained in $78.8 \mathrm{mg}$ (74\% yield) as yellowish oil. ${ }^{1} \mathrm{H}$ NMR analysis revealed that the dr value was 17:1. HPLC analysis: [Chiralcel AD-H, $15 \%{ }^{i} \mathrm{PrOH} / \mathrm{hexane}, 1.0 \mathrm{~mL} / \mathrm{min}, 254 \mathrm{~nm}$; major diastereomer: $\mathrm{t}_{\mathrm{r}}$ (major) $=10.97$ $\min , \mathrm{t}_{\mathrm{r}}($ minor $\left.)=13.48 \mathrm{~min}\right]$ gave the isomeric composition of major diastereomer: $97 \%$ ee; $[\alpha]^{25} \mathrm{D}=+9.9\left(\mathrm{c}=1.00, \mathrm{CHCl}_{3}\right) .{ }^{1} \mathrm{H} \mathrm{NMR}\left(400 \mathrm{MHz}, \mathrm{CDCl}_{3}\right): \delta 8.77(\mathrm{~s}, 1 \mathrm{H}), 7.44-7.42(\mathrm{~m}$, $1 \mathrm{H}), 7.40-7.34(\mathrm{~m}, 3 \mathrm{H}), 7.33-7.24(\mathrm{~m}, 3 \mathrm{H}), 6.85(\mathrm{~d}, J=8.4 \mathrm{~Hz}, 1 \mathrm{H}), 6.56(\mathrm{~d}, J=15.6 \mathrm{~Hz}, 1 \mathrm{H})$, 6.38-6.31 (m, 1H), 5.37-5.32 (m, 1H), 5.03-4.95 (m, 2H), $4.34(\mathrm{t}, J=11.6 \mathrm{~Hz}, 1 \mathrm{H}), 4.20(\mathrm{ABd}, J=$ 11.6, 3.6 Hz, 1H), 3.52-3.47 (m, 1H), 2.68, 2.57 (ABd, $J=13.6,7.6 \mathrm{~Hz}, 2 \mathrm{H}) ;{ }^{13} \mathrm{C} \mathrm{NMR}(100 \mathrm{MHz}$, $\left.\mathrm{CDCl}_{3}\right): \delta 178.9,139.9,136.8,135.9,132.0,131.8,130.3,128.6,128.3,126.9,126.8,121.9,120.6$, 115.8, 111.7, 76.1, 54.4, 48.7, 40.1; IR (ATR): 1710, 1557, 1476, 1377, 1287, 1179, 910, 775, 760, $737 \mathrm{~cm}^{-1}$; HRMS (ESI): Exact mass calcd for $\mathrm{C}_{21} \mathrm{H}_{19} \mathrm{~N}_{2} \mathrm{NaO}_{3} \mathrm{Br}[\mathrm{M}+\mathrm{Na}]^{+}$: 449.0471, Found: 449.0471 .

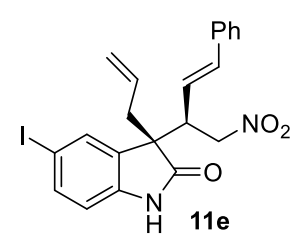

Product 11e was obtained in $88.9 \mathrm{mg}$ (75\% yield) as yellowish oil. ${ }^{1} \mathrm{H} \mathrm{NMR}$ analysis revealed that the dr value was 17:1. HPLC analysis: [Chiralcel AD-H, $15 \%{ }^{i} \mathrm{PrOH} /$ hexane, $1.0 \mathrm{~mL} / \mathrm{min}, 254 \mathrm{~nm}$; major diastereomer: $\mathrm{t}_{\mathrm{r}}$ (major) $=12.33$ $\min , \mathrm{t}_{\mathrm{r}}($ minor $\left.)=17.76 \mathrm{~min}\right]$ gave the isomeric composition of major diastereomer: $98 \%$ ee; $[\alpha]^{25} \mathrm{D}=+8.6\left(\mathrm{c}=1.00, \mathrm{CHCl}_{3}\right) .{ }^{1} \mathrm{H} \mathrm{NMR}\left(400 \mathrm{MHz}, \mathrm{CDCl}_{3}\right): \delta 8.70(\mathrm{~s}, 1 \mathrm{H}), 7.62-7.56(\mathrm{~m}$, 2H), 7.37-7.25 (m, 5H), $6.75(\mathrm{~d}, J=8.0 \mathrm{~Hz}, 1 \mathrm{H}), 6.56(\mathrm{~d}, J=15.6 \mathrm{~Hz}, 1 \mathrm{H}), 6.37-6.30(\mathrm{~m}, 1 \mathrm{H})$, 5.36-5.32 (m, 1H), 5.03-4.94 (m, 2H), $4.34(\mathrm{t}, J=11.6 \mathrm{~Hz}, 1 \mathrm{H}), 4.21(\mathrm{ABd}, J=11.6,3.6 \mathrm{~Hz}, 1 \mathrm{H})$, 3.51-3.46 (m, 1H), 2.67, $2.57(\mathrm{ABd}, J=13.6,8.0 \mathrm{~Hz}, 2 \mathrm{H}) ;{ }^{13} \mathrm{C}$ NMR (100 MHz, $\left.\mathrm{CDCl}_{3}\right): \delta 178.6$, $140.5,138.0,136.8,135.9,132.4,132.1,130.3,128.6,128.3,126.8,121.9,120.5,112.2,85.6,76.1$, 54.2, 48.7, 40.1; IR (ATR): 1713, 1558, 1474, 1287, 1265, 1180, 910, 775, $735 \mathrm{~cm}^{-1}$; HRMS (ESI): Exact mass calcd for $\mathrm{C}_{21} \mathrm{H}_{19} \mathrm{~N}_{2} \mathrm{NaO}_{3} \mathrm{I}[\mathrm{M}+\mathrm{Na}]^{+}$: 497.0333, Found: 497.0337.

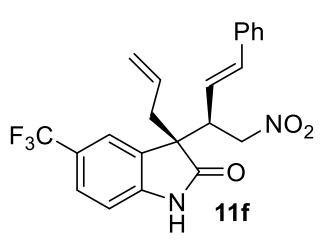

Product 11f was obtained in $67.6 \mathrm{mg}$ (65\% yield) as yellowish oil. ${ }^{1} \mathrm{H}$ NMR analysis revealed that the dr value was 18:1. HPLC analysis: [Chiralcel OD-H, $15 \%{ }^{i} \mathrm{PrOH} / \mathrm{h}$ exane, $1.0 \mathrm{~mL} / \mathrm{min}, 254 \mathrm{~nm}$; major diastereomer: $\mathrm{t}_{\mathrm{r}}$ (major) $=7.53$ min, $\mathrm{t}_{\mathrm{r}}($ minor $\left.)=6.28 \mathrm{~min}\right]$ gave the isomeric composition of major diastereomer: $97 \%$ ee; $[\alpha]^{25} \mathrm{D}=+18.6\left(\mathrm{c}=1.00, \mathrm{CHCl}_{3}\right) .{ }^{1} \mathrm{H} \mathrm{NMR}\left(400 \mathrm{MHz}, \mathrm{CDCl}_{3}\right): \delta 8.79(\mathrm{~s}, 1 \mathrm{H}), 7.59-7.50(\mathrm{~m}$, 
2H), 7.38-7.26 (m, 5H), $7.05(\mathrm{~d}, J=8.0 \mathrm{~Hz}, 1 \mathrm{H}), 6.58(\mathrm{~d}, J=16.0 \mathrm{~Hz}, 1 \mathrm{H}), 6.38-6.31(\mathrm{~m}, 1 \mathrm{H})$, 5.36-5.31 (m, 1H), 5.02-4.95 (m, 2H), $4.36(\mathrm{t}, J=10.8 \mathrm{~Hz}, 1 \mathrm{H}), 4.25(\mathrm{ABd}, J=11.6,3.6 \mathrm{~Hz}, 1 \mathrm{H})$, 3.57-3.52 (m, 1H), 2.71, $2.64(\mathrm{ABd}, J=13.6,7.6 \mathrm{~Hz}, 2 \mathrm{H}) ;{ }^{13} \mathrm{C} \mathrm{NMR}\left(125 \mathrm{MHz}, \mathrm{CDCl}_{3}\right): \delta 179.2$, 143.8, 137.1, 135.8, 130.3, 130.1, 128.6, 128.4, 126.8 (q, $J=3.75 \mathrm{~Hz}), 126.8,125.6(\mathrm{q}, J=62.5,32.5$ $\mathrm{Hz}), 124.1(\mathrm{q}, J=270.0 \mathrm{~Hz}), 120.9(\mathrm{q}, J=3.75 \mathrm{~Hz}), 110.1,76.0,54.2,48.5,39.9 ;{ }^{19} \mathrm{~F}$ NMR $(376 \mathrm{MHz}$, $\mathrm{CDCl}_{3}$ ): $\delta$-61.50; IR (ATR): 1717, 1557, 1450, 1329, 1242, 1125, 910, 880, $762 \mathrm{~cm}^{-1}$; HRMS (ESI): Exact mass calcd for $\mathrm{C}_{22} \mathrm{H}_{19} \mathrm{~N}_{2} \mathrm{NaO}_{3} \mathrm{~F}_{3}[\mathrm{M}+\mathrm{Na}]^{+}: 439.1240$, Found: 439.1249 .

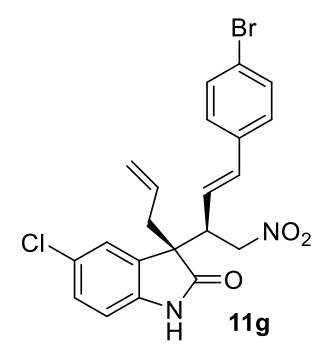

Product $11 \mathrm{~g}$ was obtained in $88.6 \mathrm{mg}$ (77\% yield) as yellowish oil. ${ }^{1} \mathrm{H}$ NMR analysis revealed that the dr value was 12:1. HPLC analysis: [Chiralcel AD-H, $15 \%{ }^{i} \mathrm{PrOH} /$ hexane, $1.0 \mathrm{~mL} / \mathrm{min}, 254 \mathrm{~nm}$; major diastereomer: $\mathrm{t}_{\mathrm{r}}$ (major) $=14.37$ $\min , \mathrm{t}_{\mathrm{r}}($ minor $\left.)=18.56 \mathrm{~min}\right]$ gave the isomeric composition of major diastereomer: $98 \%$ ee; $[\alpha]^{25}{ }_{\mathrm{D}}=+20.8\left(\mathrm{c}=1.00, \mathrm{CHCl}_{3}\right) .{ }^{1} \mathrm{H} \mathrm{NMR}\left(400 \mathrm{MHz}, \mathrm{CDCl}_{3}\right): \delta 8.59(\mathrm{~s}$, $1 \mathrm{H}), 7.45-7.42(\mathrm{~m}, 2 \mathrm{H}), 7.30-7.21(\mathrm{~m}, 4 \mathrm{H}), 6.88(\mathrm{~d}, J=8.4 \mathrm{~Hz} 1 \mathrm{H}), 6.51(\mathrm{~d}, J=15.6 \mathrm{~Hz} 1 \mathrm{H})$, 6.41-6.35 (m, 1H), 5.37-5.32 (m, 1H), 5.04-4.95 (m, 2H), $4.35(\mathrm{t}, J=11.6 \mathrm{~Hz}, 1 \mathrm{H}), 4.22(\mathrm{ABd}, J=$ 11.6, 3.6 Hz, 1H), 3.51-3.48 (m, 1H), 2.67, $2.57(\mathrm{ABd}, J=13.6,7.6 \mathrm{~Hz}, 2 \mathrm{H}) ;{ }^{13} \mathrm{C}$ NMR $(100 \mathrm{MHz}$, $\left.\mathrm{CDCl}_{3}\right): \delta 178.7,139.2,135.6,134.8,131.8,131.3,130.2,129.2,128.7,128.3,124.1,122.8,122.2$, 120.6, 111.2, 76.0, 54.2, 48.6, 40.1; IR (ATR): 1713, 1557, 1478, 1246, 1180, 1009, 910, 764, 733 $\mathrm{cm}^{-1}$; HRMS (ESI): Exact mass calcd for $\mathrm{C}_{21} \mathrm{H}_{18} \mathrm{~N}_{2} \mathrm{NaO}_{3} \mathrm{ClBr}$ [M+Na] $]^{+}$: 483.0082, Found: 483.0093.

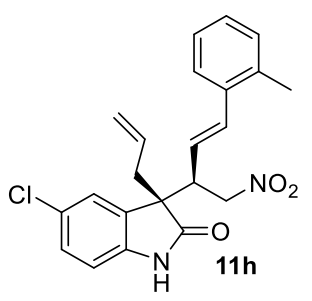

Product $11 \mathrm{~h}$ was obtained in $71.3 \mathrm{mg}\left(72 \%\right.$ yield) as yellowish oil. ${ }^{1} \mathrm{H}$ NMR analysis revealed that the dr value was >20:1. HPLC analysis: [Chiralcel AD-H, $15 \%{ }^{i} \mathrm{PrOH} / \mathrm{hexane}, 1.0 \mathrm{~mL} / \mathrm{min}, 254 \mathrm{~nm}$; major diastereomer: $\mathrm{t}_{\mathrm{r}}$ (major) $=6.82$ $\left.\min , \mathrm{t}_{\mathrm{r}}(\operatorname{minor})=10.12 \mathrm{~min}\right]$ gave the isomeric composition of major diastereomer: $97 \%$ ee; $[\alpha]^{25} \mathrm{D}=+32.4\left(\mathrm{c}=1.00, \mathrm{CHCl}_{3}\right) .{ }^{1} \mathrm{H} \mathrm{NMR}\left(400 \mathrm{MHz}, \mathrm{CDCl}_{3}\right): \delta 8.52(\mathrm{~s}$, $1 \mathrm{H}), 7.38-7.37(\mathrm{~m}, 1 \mathrm{H}), 7.28-7.26(\mathrm{~m}, 2 \mathrm{H}), 7.18-7.14(\mathrm{~m}, 3 \mathrm{H}), 6.88(\mathrm{~d}, J=8.8 \mathrm{~Hz}, 1 \mathrm{H}), 6.77(\mathrm{~d}, J=$ $15.6 \mathrm{~Hz}, 1 \mathrm{H}), 6.23-6.17(\mathrm{~m}, 1 \mathrm{H}), 5.40-5.38(\mathrm{~m}, 1 \mathrm{H}), 5.06-4.96(\mathrm{~m}, 2 \mathrm{H}), 4.39(\mathrm{t}, J=11.6 \mathrm{~Hz}, 1 \mathrm{H}), 4.31$ $(\mathrm{ABd}, J=11.6,3.6 \mathrm{~Hz}, 1 \mathrm{H}), 3.55-3.50(\mathrm{~m}, 1 \mathrm{H}), 2.70,2.61(\mathrm{ABd}, J=13.6,7.6 \mathrm{~Hz}, 2 \mathrm{H}), 2.30(\mathrm{~s}, 3 \mathrm{H})$; ${ }^{13} \mathrm{C}$ NMR (100 MHz, $\left.\mathrm{CDCl}_{3}\right): \delta 178.7,139.3,135.7,135.4,135.2,131.4,130.3,130.2,129.1,128.6$, 128.1, 126.3, 126.1, 124.2, 123.4, 120.5, 111.1, 76.2, 54.3, 48.8, 39.9, 19.7; IR (ATR): 1713, 1557, 1478, 1292, 1244, 1180, 912, 756, $737 \mathrm{~cm}^{-1}$; HRMS (ESI): Exact mass calcd for $\mathrm{C}_{22} \mathrm{H}_{21} \mathrm{~N}_{2} \mathrm{NaO}_{3} \mathrm{Cl}$ $[\mathrm{M}+\mathrm{Na}]^{+}:$419.1133, Found: 419.1141. 


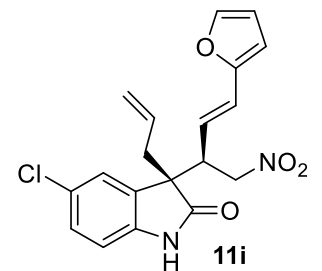

Product 11i was obtained in $72.5 \mathrm{mg}$ (78\% yield) as yellowish oil. ${ }^{1} \mathrm{H}$ NMR analysis revealed that the dr value was 14:1. HPLC analysis: [Chiralcel OD-H, $15 \%{ }^{i} \mathrm{PrOH} / \mathrm{hexane}, 1.0 \mathrm{~mL} / \mathrm{min}, 254 \mathrm{~nm}$; major diastereomer: $\mathrm{t}_{\mathrm{r}}$ (major) $=8.07$ min, $\mathrm{t}_{\mathrm{r}}($ minor $)=7.14 \mathrm{~min}$; minor diastereomer] gave the isomeric composition of major diastereomer: $97 \%$ ee; $[\alpha]^{25} \mathrm{D}=+8.4\left(\mathrm{c}=1.00, \mathrm{CHCl}_{3}\right) .{ }^{1} \mathrm{H} \mathrm{NMR}\left(400 \mathrm{MHz}, \mathrm{CDCl}_{3}\right): \delta 8.84(\mathrm{~s}$, $1 \mathrm{H}), 7.34-7.24(\mathrm{~m}, 3 \mathrm{H}), 6.91(\mathrm{~d}, J=8.4 \mathrm{~Hz}, 1 \mathrm{H}), 6.39-6.35(\mathrm{~m}, 2 \mathrm{H}), 6.28-6.24(\mathrm{~m}, 2 \mathrm{H}), 5.37-5.32(\mathrm{~m}$, $1 \mathrm{H}), 5.04-4.94(\mathrm{~m}, 2 \mathrm{H}), 4.36(\mathrm{t}, J=11.6 \mathrm{~Hz}, 1 \mathrm{H}), 4.26(\mathrm{ABd}, J=12.0,3.6 \mathrm{~Hz}, 1 \mathrm{H}), 3.48-3.44(\mathrm{~m}, 1 \mathrm{H})$, 2.69, $2.58(\mathrm{ABd}, J=13.6,7.6 \mathrm{~Hz}, 2 \mathrm{H}) ;{ }^{13} \mathrm{C} \mathrm{NMR}\left(100 \mathrm{MHz}, \mathrm{CDCl}_{3}\right): \delta 178.9,151.4,142.5,139.4$, 131.3, 130.3, 129.1, 128.6, 124.7, 124.1, 120.5, 120.3, 111.4, 111.3, 109.3, 76.0, 54.4, 48.5, 39.9; IR (ATR): 1713, 1558, 1480, 1377, 1246, 1126, 880, 766, $735 \mathrm{~cm}^{-1}$; HRMS (ESI): Exact mass calcd for $\mathrm{C}_{19} \mathrm{H}_{17} \mathrm{~N}_{2} \mathrm{NaO}_{3} \mathrm{Cl}[\mathrm{M}+\mathrm{Na}]^{+}: 395.0769$, Found: 395.0779.

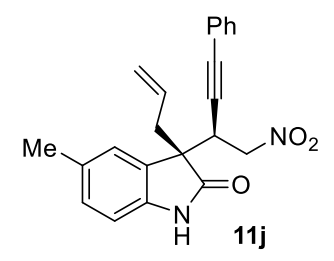

The reaction was conducted at $25{ }^{\circ} \mathrm{C}$ and 10.0 equiv of allyltrimethylsilane was used in this case. Product $\mathbf{1 1} \mathbf{j}$ was obtained in $52.2 \mathrm{mg}$ (58\% yield) as yellowish solid. ${ }^{1} \mathrm{H}$ NMR analysis revealed that the $\mathrm{dr}$ value was $3: 1$. HPLC analysis: [Chiralcel AD-H, 10\% ${ }^{i} \mathrm{PrOH} / \mathrm{hexane}, 1.0 \mathrm{~mL} / \mathrm{min}, 230 \mathrm{~nm}$; major diastereomer: $\mathrm{t}_{\mathrm{r}}($ major $)=11.79$ min, $\mathrm{t}_{\mathrm{r}}($ minor $)=15.02 \mathrm{~min}$; minor diastereomer: $\mathrm{t}_{\mathrm{r}}($ minor $)=8.07$ min, $\mathrm{t}_{\mathrm{r}}($ major $)=$ $10.27 \mathrm{~min}]$ gave the isomeric composition of major diastereomer: $92 \%$ ee; $[\alpha]^{25} \mathrm{D}=-2.1(\mathrm{c}=1.01$, $\mathrm{CH}_{2} \mathrm{Cl}_{2}$ ). Mp 58-60 ${ }^{\circ} \mathrm{C} .{ }^{1} \mathrm{H}$ NMR $\left(400 \mathrm{MHz}, \mathrm{CDCl}_{3}\right): \delta$ 8.04-7.79 (m, $\left.1 \mathrm{H}\right), 7.42-7.40(\mathrm{~m}, 2 \mathrm{H})$, 7.33-7.28 (m, 3H), 7.24 (s, 1H), 7.09-7.07 (m, 1H), 6.82-6.79 (m, 1H), 5.48-5.37 (m, 1H), 5.12-5.08 (m, 1H), 5.00-4.97 (m, 1H), 4.83-4.78 (m, 1H), 4.65-4.59 (m, 1H), 3.92-3.88 (m, 1H), 2.89, 2.77 $(\mathrm{ABd}, J=13.6, J=6.8 \mathrm{~Hz}, 2 \mathrm{H}), 2.36(\mathrm{~s}, 3 \mathrm{H}) ;{ }^{13} \mathrm{C} \mathrm{NMR}\left(100 \mathrm{MHz}, \mathrm{CDCl}_{3}\right): \delta 178.5,138.3,132.5$, 132.0, 131.2, 129.6, 129.4, 128.8, 128.4, 125.3, 122.4, 120.2, 110.0, 86.8, 83.6, 75.3, 53.0, 38.1, 37.8, 21.44; IR (ATR): 2921, 1707, 1624, 1555, 1433, 1326, 1235, $1159 \mathrm{~cm}^{-1}$; HRMS (ESI): Exact mass calcd for $\mathrm{C}_{22} \mathrm{H}_{24} \mathrm{~N}_{3} \mathrm{O}_{3}\left[\mathrm{M}+\mathrm{NH}_{4}\right]^{+}$: 378.1812, Found: 378.1820 .

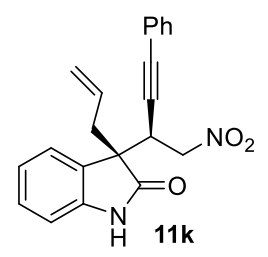

10.0 equiv of allyltrimethylsilane was used in this case. Product $11 \mathbf{k}$ was obtained in $55.4 \mathrm{mg}$ (64\% yield) as yellowish solid. ${ }^{1} \mathrm{H}$ NMR analysis revealed that the dr value was 5:1. HPLC analysis: [Chiralcel AD-H, $15 \%{ }^{i} \mathrm{PrOH} / \mathrm{hexane}, 1.0 \mathrm{~mL} / \mathrm{min}, 254 \mathrm{~nm}$; major diastereomer: $\mathrm{t}_{\mathrm{r}}($ major $)=11.56 \mathrm{~min}, \mathrm{t}_{\mathrm{r}}($ minor $\left.)=13.54 \mathrm{~min}\right]$ gave the isomeric composition of major diastereomer: $97 \%$ ee; $[\alpha]^{25} \mathrm{D}=+19.1\left(\mathrm{c}=1.00, \mathrm{CHCl}_{3}\right)$. $\mathrm{Mp} 52-53{ }^{\circ} \mathrm{C}$. ${ }^{1} \mathrm{H}$ NMR (400 MHz, $\left.\mathrm{CDCl}_{3}\right): \delta 8.73(\mathrm{~s}, 1 \mathrm{H}), 7.44-7.39(\mathrm{~m}, 3 \mathrm{H}), 7.32-7.26(\mathrm{~m}, 4 \mathrm{H}), 7.12-7.08(\mathrm{~m}, 1 \mathrm{H})$, 
$6.96(\mathrm{~d}, J=7.6 \mathrm{~Hz}, 1 \mathrm{H}), 5.43-5.41(\mathrm{~m}, 1 \mathrm{H}), 5.12-5.07(\mathrm{~m}, 1 \mathrm{H}), 4.99-4.96(\mathrm{~m}, 1 \mathrm{H}), 4.81(\mathrm{ABd}, J=$ 12.4, 4.4 Hz, 1H), 4.62 (ABd, $J=12.8,6.4 \mathrm{~Hz}, 1 \mathrm{H}), 3.92$ (dd, $J=10.8,4.4 \mathrm{~Hz}, 1 \mathrm{H}), 2.93,2.77$ (ABd, $J=13.6,6.8 \mathrm{~Hz}, 2 \mathrm{H}) ;{ }^{13} \mathrm{C} \mathrm{NMR}\left(100 \mathrm{MHz}, \mathrm{CDCl}_{3}\right): \delta 178.7,139.8,132.0,132.0,131.6,130.5,129.0$, 128.5, 128.0, 121.9, 120.8, 115.7, 112.0, 87.2, 83.1, 74.9, 53.3, 37.5, 37.4; IR (ATR): 1714, 1558, 1472, 1288, 1184, 910, 779, 760, $735 \mathrm{~cm}^{-1}$; HRMS (ESI): Exact mass calcd for $\mathrm{C}_{21} \mathrm{H}_{18} \mathrm{~N}_{2} \mathrm{NaO}_{3}$ $[\mathrm{M}+\mathrm{Na}]^{+}: 369.1210$, Found: 369.1216.

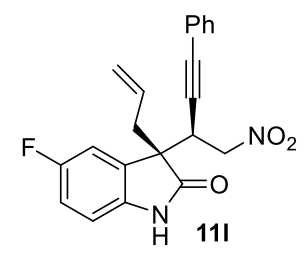

Product $11 \mathrm{l}$ was obtained in $80.1 \mathrm{mg}$ (88\% yield) as yellowish solid. ${ }^{1} \mathrm{H}$ NMR analysis revealed that the dr value was 4:1. HPLC analysis: [Chiralcel AD-H, 15\% ${ }^{i} \mathrm{PrOH} /$ hexane, $1.0 \mathrm{~mL} / \mathrm{min}, 230 \mathrm{~nm}$; major diastereomer: $\mathrm{t}_{\mathrm{r}}$ (major) $=8.15 \mathrm{~min}, \mathrm{t}_{\mathrm{r}}$ $($ minor $)=10.28 \mathrm{~min} ;$ minor diastereomer: $\mathrm{t}_{\mathrm{r}}($ minor $)=7.13 \mathrm{~min}, \mathrm{t}_{\mathrm{r}}($ major $)=9.30$ $\min ]$ gave the isomeric composition of major diastereomer: $96 \%$ ee; $[\alpha]^{25} \mathrm{D}=+49.0\left(\mathrm{c}=1.08, \mathrm{CH}_{2} \mathrm{Cl}_{2}\right)$. Mp 60-62 ${ }^{\circ} \mathrm{C} .{ }^{1} \mathrm{H}$ NMR (400 MHz, $\left.\mathrm{CDCl}_{3}\right): \delta 9.03(\mathrm{~s}, 1 \mathrm{H}), 7.42-7.40(\mathrm{~m}, 2 \mathrm{H}), 7.36-7.30(\mathrm{~m}, 3 \mathrm{H})$, 7.24-7.21 (m, 1H), 7.01-6.96 (m, 1H), 6.93-6.90 (m, 1H), 5.46-5.36 (m, 1H), 5.12-5.07 (m, 1H), 5.01-4.98 (m, 1H), $4.91(\mathrm{ABd}, J=12.8,4.4 \mathrm{~Hz}, 1 \mathrm{H}), 4.67-4.61(\mathrm{~m}, 1 \mathrm{H}), 3.90(\mathrm{ABd}, J=10.4,4.4 \mathrm{~Hz}$, $1 \mathrm{H}), 2.91,2.76(\mathrm{ABd}, J=13.2,6.8 \mathrm{~Hz}, 2 \mathrm{H}) ;{ }^{13} \mathrm{C} \mathrm{NMR}\left(100 \mathrm{MHz}, \mathrm{CDCl}_{3}\right): \delta 178.9,159.2\left(\mathrm{~d}, J_{\mathrm{C}-\mathrm{F}}=\right.$ $240.0 \mathrm{~Hz}), 136.7\left(\mathrm{~d}, J_{\mathrm{C}-\mathrm{F}}=2.0 \mathrm{~Hz}\right), 131.9,131.2\left(\mathrm{~d}, J_{\mathrm{C}-\mathrm{F}}=8.0 \mathrm{~Hz}\right), 130.6,129.0,128.5,121.99,120.7$, $115.8\left(\mathrm{~d}, J_{\mathrm{C}-\mathrm{F}}=23.0 \mathrm{~Hz}\right), 112.8\left(\mathrm{~d}, J_{\mathrm{C}-\mathrm{F}}=25.0 \mathrm{~Hz}\right), 111.1\left(\mathrm{~d}, J_{\mathrm{C}-\mathrm{F}}=8.0 \mathrm{~Hz}\right), 87.2,83.1,75.0,53.5$, 37.7, 37.5; ${ }^{19} \mathrm{~F}$ NMR (376 MHz, $\left.\mathrm{CDCl}_{3}\right): \delta$-119.11; IR (ATR): 1710, 1628, 1557, 1486, 1443, 1328 , 1262, $925 \mathrm{~cm}^{-1}$; HRMS (ESI): Exact mass calcd for $\mathrm{C}_{21} \mathrm{H}_{21} \mathrm{~N}_{3} \mathrm{O}_{3} \mathrm{~F}\left[\mathrm{M}+\mathrm{NH}_{4}\right]^{+}$: 382.1561, Found: 382.1569.

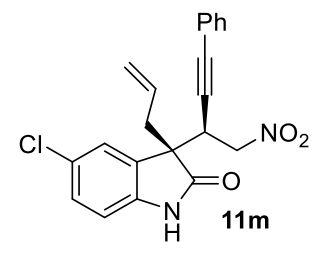

Product $11 \mathrm{~m}$ was obtained in $77.0 \mathrm{mg}$ (81\% yield) as yellowish solid. ${ }^{1} \mathrm{H}$ NMR analysis revealed that the dr value was 5:1. HPLC analysis: [Chiralcel AD-H, 15\% ${ }^{i} \mathrm{PrOH} / \mathrm{hexane}, 1.0 \mathrm{~mL} / \mathrm{min}, 254 \mathrm{~nm}$; major diastereomer: $\mathrm{t}_{\mathrm{r}}$ (major) $=7.70 \mathrm{~min}, \mathrm{t}_{\mathrm{r}}$ $($ minor $)=8.74 \mathrm{~min}]$ gave the isomeric composition of major diastereomer: $97 \%$ ee; $[\alpha]^{25} \mathrm{D}=+16.8\left(\mathrm{c}=1.00, \mathrm{CHCl}_{3}\right) . \mathrm{Mp} 66-67{ }^{\circ} \mathrm{C} .{ }^{1} \mathrm{H}$ NMR $\left(400 \mathrm{MHz}, \mathrm{CDCl}_{3}\right): \delta 8.45(\mathrm{~s}, 1 \mathrm{H}), 7.49$ $(\mathrm{d}, J=2.0 \mathrm{~Hz}, 1 \mathrm{H}), 7.43-7.41(\mathrm{~m}, 2 \mathrm{H}), 7.34-7.26(\mathrm{~m}, 4 \mathrm{H}), 6.89(\mathrm{~d}, J=8.4 \mathrm{~Hz}, 1 \mathrm{H}), 5.42-5.35(\mathrm{~m}, 1 \mathrm{H})$, 5.12-5.08 (m, 1H), 5.01-4.94 (m, 2H), $4.65(\mathrm{ABd}, J=12.8,11.6 \mathrm{~Hz}, 1 \mathrm{H}), 3.88(\mathrm{ABd}, J=10.0,4.0 \mathrm{~Hz}$, $1 \mathrm{H}), 2.90,2.74(\mathrm{ABd}, J=13.6,6.8 \mathrm{~Hz}, 2 \mathrm{H}) ;{ }^{13} \mathrm{C} \mathrm{NMR}\left(125 \mathrm{MHz}, \mathrm{CDCl}_{3}\right): \delta 178.2,139.1,131.8$, 131.1, 130.4, 129.2, 128.9, 128.4, 128.3, 125.2, 121.8, 120.7, 111.2, 87.1, 83.0, 74.8, 53.1, 37.4, 37.3; IR (ATR): 1715, 1620, 1558, 1478, 1443, 1375, 1179, $910 \mathrm{~cm}^{-1}$; HRMS (ESI): Exact mass calcd for 
$\mathrm{C}_{21} \mathrm{H}_{17} \mathrm{~N}_{2} \mathrm{NaO}_{3} \mathrm{Cl}[\mathrm{M}+\mathrm{Na}]^{+}:$403.0820, Found: 403.0822 .

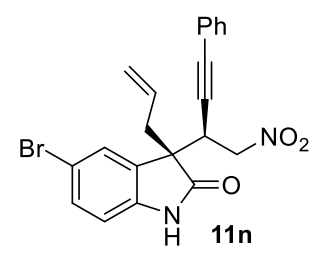

Product 11n was obtained in $82.7 \mathrm{mg}$ (78\% yield) as yellowish solid. ${ }^{1} \mathrm{H}$ NMR analysis revealed that the dr value was 5:1. HPLC analysis: [Chiralcel AD-H, 15\% ${ }^{i} \mathrm{PrOH} /$ hexane, $1.0 \mathrm{~mL} / \mathrm{min}, 254 \mathrm{~nm}$; major diastereomer: $\mathrm{t}_{\mathrm{r}}$ (major) $=7.90 \mathrm{~min}, \mathrm{t}_{\mathrm{r}}$ $($ minor $)=8.83 \mathrm{~min}]$ gave the isomeric composition of major diastereomer: $97 \%$ ee; $[\alpha]^{25} \mathrm{D}=+45.1\left(\mathrm{c}=1.00, \mathrm{CHCl}_{3}\right) . \mathrm{Mp} 74-75{ }^{\circ} \mathrm{C} .{ }^{1} \mathrm{H} \mathrm{NMR}\left(400 \mathrm{MHz}, \mathrm{CDCl}_{3}\right): \delta 9.17(\mathrm{~s}, 1 \mathrm{H})$, 7.62-7.61 (m, 1H), 7.43-7.31 (m, 6H), $6.87(\mathrm{~d}, J=8.4 \mathrm{~Hz}, 1 \mathrm{H}), 5.40-5.35(\mathrm{~m}, 1 \mathrm{H}), 5.12-5.07(\mathrm{~m}, 1 \mathrm{H})$, 5.00-4.98 (m, 1H), $4.92(\mathrm{ABd}, J=12.8,4.0 \mathrm{~Hz}, 1 \mathrm{H}), 4.64(\mathrm{ABd}, J=12.8,10.4 \mathrm{~Hz}, 1 \mathrm{H}), 3.88(\mathrm{ABd}, J$ $=10.4,4.4 \mathrm{~Hz}, 1 \mathrm{H}), 2.90,2.74(\mathrm{ABd}, J=13.6,6.8 \mathrm{~Hz}, 2 \mathrm{H}) ;{ }^{13} \mathrm{C} \mathrm{NMR}\left(100 \mathrm{MHz}, \mathrm{CDCl}_{3}\right): \delta 178.6$, 139.7, 132.1, 131.9, 131.5, 130.4, 128.9, 128.4, 127.9, 121.8, 120.7, 115.6, 111.9, 87.1, 83.0, 74.8, 53.2, 37.4, 37.3; IR (ATR): 1711, 1557, 1474, 1225, 1179, 910, 777, 758, $734 \mathrm{~cm}^{-1}$; HRMS (ESI): Exact mass calcd for $\mathrm{C}_{21} \mathrm{H}_{18} \mathrm{~N}_{2} \mathrm{O}_{3} \mathrm{Br}[\mathrm{M}+\mathrm{H}]^{+}$: 425.0495, Found: 425.0493 .

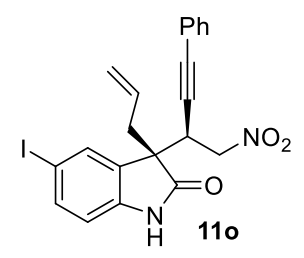

Product 110 was obtained in $88.5 \mathrm{mg}$ (75\% yield) as yellowish solid. ${ }^{1} \mathrm{H}$ NMR analysis revealed that the dr value was 8:1. HPLC analysis: [Chiralcel AD-H, 15\% ${ }^{i} \mathrm{PrOH} / \mathrm{hexane}, 1.0 \mathrm{~mL} / \mathrm{min}, 254 \mathrm{~nm}$; major diastereomer: $\mathrm{t}_{\mathrm{r}}$ (major) $=8.62 \mathrm{~min}$, $\mathrm{t}_{\mathrm{r}}$ $(\operatorname{minor})=9.78 \mathrm{~min}]$ gave the isomeric composition of major diastereomer: $99 \%$ ee; $[\alpha]^{25} \mathrm{D}=+5.5\left(\mathrm{c}=1.00, \mathrm{CHCl}_{3}\right) . \mathrm{Mp} 88-90{ }^{\circ} \mathrm{C} .{ }^{1} \mathrm{H} \mathrm{NMR}\left(400 \mathrm{MHz}, \mathrm{CDCl}_{3}\right): \delta 8.11(\mathrm{~s}, 1 \mathrm{H}), 7.83-7.82$ (m, 1H), 7.63-7.61 (m, 1H), 7.46-7.33 (m, 5H), $6.74(\mathrm{~d}, J=8.0 \mathrm{~Hz}, 1 \mathrm{H}), 5.38-5.34(\mathrm{~m}, 1 \mathrm{H}), 5.13-5.08$ (m, 1H), 5.02-4.98 (m, 2H), 4.65 (ABd, $J=12.810 .4 \mathrm{~Hz}, 1 \mathrm{H}), 3.86(\mathrm{ABd}, J=10.4,4.0 \mathrm{~Hz}, 1 \mathrm{H}), 2.89$, $2.71(\mathrm{ABd}, J=13.2,6.4 \mathrm{~Hz}, 2 \mathrm{H}) ;{ }^{13} \mathrm{C} \mathrm{NMR}\left(125 \mathrm{MHz}, \mathrm{CDCl}_{3}\right): \delta 177.4,140.1,138.0,133.8,131.9$, 131.85, 130.4, 128.9, 128.4, 121.8, 120.7, 112.0, 87.1, 85.3, 83.0, 74.8, 52.8, 37.5, 37.2; IR (ATR): $1717,1558,1288,1248,910,779,760,737 \mathrm{~cm}^{-1}$; HRMS (ESI): Exact mass calcd for $\mathrm{C}_{21} \mathrm{H}_{17} \mathrm{~N}_{2} \mathrm{NaO}_{3} \mathrm{I}$ $[\mathrm{M}+\mathrm{Na}]^{+}:$495.0176, Found: 495.0176.

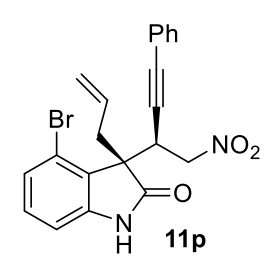

The reaction was conducted at $0{ }^{\circ} \mathrm{C}$ and 10.0 equiv of allyltrimethylsilane was used in this case. Product 11p was obtained in $68.9 \mathrm{mg}$ (65\% yield) as yellowish solid. ${ }^{1} \mathrm{H}$ NMR analysis revealed that the dr value was 7:1. HPLC analysis: [Chiralcel AD-H, $15 \%{ }^{i} \mathrm{PrOH} / \mathrm{hexane}, 1.0 \mathrm{~mL} / \mathrm{min}, 230 \mathrm{~nm}$; major diastereomer: $\mathrm{t}_{\mathrm{r}}$ (major) $=14.41 \mathrm{~min}$, $\mathrm{t}_{\mathrm{r}}($ minor $)=18.57 \mathrm{~min}$; minor diastereomer: $\mathrm{t}_{\mathrm{r}}(\operatorname{minor})=11.22 \mathrm{~min}, \mathrm{t}_{\mathrm{r}}($ major $\left.)=12.09 \mathrm{~min}\right]$ gave the isomeric composition of major diastereomer: $96 \%$ ee; $[\alpha]^{25} \mathrm{D}=-80.8\left(\mathrm{c}=0.33, \mathrm{CH}_{2} \mathrm{Cl}_{2}\right) . \mathrm{Mp} 70-72{ }^{\circ} \mathrm{C}$. 
${ }^{1} \mathrm{H}$ NMR (400 MHz, $\left.\mathrm{CDCl}_{3}\right): \delta 9.21(\mathrm{~s}, 1 \mathrm{H})$, 7.49-7.46 (m, 2H), 7.33-7.28 (m, 3H), 7.21-7.19 (m, 1H), 7.04-7.00 (m, 1H), 6.91-6.89 (m, 1H), 5.35-5.25 (m, 1H), 5.13-5.08 (m, 1H), 4.89-4.86 (m, 1H), 4.76 $(\mathrm{ABd}, J=10.4,4.0 \mathrm{~Hz}, 1 \mathrm{H}), 4.49-4.43(\mathrm{~m}, 1 \mathrm{H}), 4.04(\mathrm{ABd}, J=12.4,4.0 \mathrm{~Hz}, 1 \mathrm{H}), 3.34,3.20(\mathrm{ABd}, J$ $=13.6,7.6 \mathrm{~Hz}, 2 \mathrm{H}) ;{ }^{13} \mathrm{C} \mathrm{NMR}\left(100 \mathrm{MHz}, \mathrm{CDCl}_{3}\right): \delta 177.3,143.4,132.1,131.1,130.9,128.8,128.4$, 127.6, 126.1, 122.6, 119.9, 119.4, 109.7, 87.4, 82.6, 76.0, 55.6, 37.3, 35.6; IR (ATR): 1716, 1613, 1579, 1490, 1375, 1304, 1234, $1174 \mathrm{~cm}^{-1}$; HRMS (ESI): Exact mass calcd for $\mathrm{C}_{21} \mathrm{H}_{21} \mathrm{~N}_{3} \mathrm{O}_{3}{ }^{79} \mathrm{Br}$ $\left[\mathrm{M}+\mathrm{NH}_{4}\right]^{+}:$442.0761, Found: 442.0766.

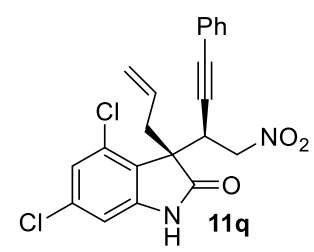

Product 11q was obtained in $94.2 \mathrm{mg}$ (91\% yield) as yellowish solid. ${ }^{1} \mathrm{H}$ NMR analysis revealed that the dr value was 9:1. HPLC analysis: [Chiralcel AD-H, 5\% ${ }^{i} \mathrm{PrOH} /$ hexane, $1.0 \mathrm{~mL} / \mathrm{min}, 230 \mathrm{~nm}$; major diastereomer: $\mathrm{t}_{\mathrm{r}}$ (major) $=30.16 \mathrm{~min}$, $\mathrm{t}_{\mathrm{r}}($ minor $)=59.56 \min ;$ minor diastereomer: $\mathrm{t}_{\mathrm{r}}($ minor $)=33.20$ min, $\mathrm{t}_{\mathrm{r}}($ major $)=$ $25.41 \mathrm{~min}]$ gave the isomeric composition of major diastereomer: $96 \%$ ee; $[\alpha]^{25}=-120.4(\mathrm{c}=0.37$, $\mathrm{CH}_{2} \mathrm{Cl}_{2}$ ). Mp 134-136 ${ }^{\circ} \mathrm{C} .{ }^{1} \mathrm{H}$ NMR (400 MHz, $\left.\mathrm{CDCl}_{3}\right): \delta$ 9.07-8.67 (m, 1H), 7.44-7.42 (m, 2H), 7.34-7.28 (m, 3H), 7.08-7.07 (m, 1H), 6.87-6.85 (m, 1H), 5.35-5.26 (m, 1H), 5.13-5.09 (m, 1H), 4.94-4.92 (m, 1H), 4.56-4.47 (m, 2H), 4.20-4.16 (m, 1H), 3.20-3.18 (m, 2H); ${ }^{13} \mathrm{C}$ NMR (100 MHz, $\left.\mathrm{CDCl}_{3}\right): \delta 177.7,143.8,136.2,132.0,131.6,130.5,128.9,128.4,124.1,123.2,122.3,120.3,110.0$, 87.5, 82.2, 76.0, 55.2, 37.3, 36.2; IR (ATR): 2236, 1732, 1697, 1580, 1490, 1433, 1341, 1227, 1175 $\mathrm{cm}^{-1}$; HRMS (ESI): Exact mass calcd for $\mathrm{C}_{21} \mathrm{H}_{20} \mathrm{~N}_{3} \mathrm{O}_{3}{ }^{35} \mathrm{Cl}_{2}\left[\mathrm{M}+\mathrm{NH}_{4}\right]^{+}$: 432.0876 , Found: 432.0882.

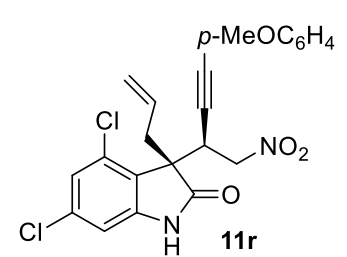

Product 11r was obtained in $92.1 \mathrm{mg}$ (83\% yield) as yellowish solid. ${ }^{1} \mathrm{H}$ NMR analysis revealed that the dr value was 9:1. HPLC analysis: [Chiralcel AD-H, $10 \%{ }^{i} \mathrm{PrOH} /$ hexane, $1.0 \mathrm{~mL} / \mathrm{min}, 230 \mathrm{~nm}$; major diastereomer: $\mathrm{t}_{\mathrm{r}}$ (major) $=25.12$ $\min , \mathrm{t}_{\mathrm{r}}($ minor $)=64.85 \mathrm{~min}$; minor diastereomer: $\mathrm{t}_{\mathrm{r}}($ major $)=19.39 \mathrm{~min}, \mathrm{t}_{\mathrm{r}}$ $($ minor $)=21.06 \mathrm{~min}]$ gave the isomeric composition of major diastereomer: $95 \%$ ee; $[\alpha]^{25} \mathrm{D}=-88.8(\mathrm{c}$ $\left.=0.70, \mathrm{CH}_{2} \mathrm{Cl}_{2}\right) \cdot \mathrm{Mp} 125-127{ }^{\circ} \mathrm{C} .{ }^{1} \mathrm{H} \mathrm{NMR}\left(400 \mathrm{MHz}, \mathrm{CDCl}_{3}\right): \delta 9.26(\mathrm{~s}, 1 \mathrm{H}), 7.35(\mathrm{ABd}, J=8.8 \mathrm{~Hz}$, 2H), $7.06(\mathrm{~d}, \mathrm{~J}=1.2 \mathrm{~Hz}, 1 \mathrm{H}), 6.87(\mathrm{~d}, \mathrm{~J}=1.6 \mathrm{~Hz}, 1 \mathrm{H}), 6.82(\mathrm{ABd}, \mathrm{J}=8.8 \mathrm{~Hz}, 2 \mathrm{H}), 5.35-5.25(\mathrm{~m}, 1 \mathrm{H})$, 5.12-5.08 (m, 1H), 4.93-4.90 (m, 1H), 4.53-4.45 (m, 2H), 4.21-4.12 (m, 1H), $3.79(\mathrm{~s}, 3 \mathrm{H}), 3.17(\mathrm{~d}, J=$ $7.2 \mathrm{~Hz}, 2 \mathrm{H}) ;{ }^{13} \mathrm{C}$ NMR $\left(100 \mathrm{MHz}, \mathrm{CDCl}_{3}\right): \delta 177.6,160.1,143.9,136.1,133.5,131.6,130.6,124.1$, 123.4, 120.3 , 114.4, 114.1, 109.9, 87.4, 80.7, 76.1, 55.4, 55.3, 37.2, 36.3; IR (ATR): 2928, 1729, 1693, 1581, 1439, 1377, 1298, $1204 \mathrm{~cm}^{-1}$; HRMS (ESI): Exact mass calcd for $\mathrm{C}_{22} \mathrm{H}_{22} \mathrm{~N}_{3} \mathrm{O}_{4}{ }^{35} \mathrm{Cl}_{2}\left[\mathrm{M}+\mathrm{NH}_{4}\right]^{+}$: 462.0982, Found: 462.0987. 


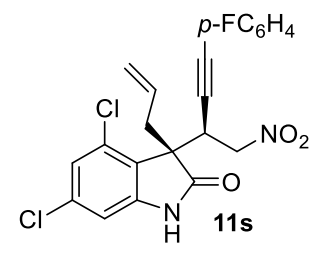

Product 11s was obtained in $94.0 \mathrm{mg}\left(87 \%\right.$ yield) as yellowish solid. ${ }^{1} \mathrm{H}$ NMR analysis revealed that the dr value was 13:1. HPLC analysis: [Chiralcel AD-H, $10 \%{ }^{i} \mathrm{PrOH} / \mathrm{hexane}, 1.0 \mathrm{~mL} / \mathrm{min}, 230 \mathrm{~nm}$; major diastereomer: $\mathrm{t}_{\mathrm{r}}$ (major) $=18.59$ $\min , t_{r}(\operatorname{minor})=33.35$ min; minor diastereomer: $t_{r}($ minor $)=14.04$ min, $t_{r}($ major $)$ $=14.84 \mathrm{~min}]$ gave the isomeric composition of major diastereomer: $97 \%$ ee; $[\alpha]^{25} \mathrm{D}=-77.7(\mathrm{c}=1.04$, $\mathrm{CH}_{2} \mathrm{Cl}_{2}$ ). Mp 128-130 ${ }^{\circ} \mathrm{C} .{ }^{1} \mathrm{H}$ NMR (400 MHz, $\left.\mathrm{CDCl}_{3}\right): \delta$ 9.02-8.79 (m, $\left.1 \mathrm{H}\right), 7.43-7.40(\mathrm{~m}, 2 \mathrm{H})$, 7.08-7.08 (m, 1H), 7.02-6.98 (m, 2H), 6.86-6.85 (m, 1H), 5.36-5.25 (m, 1H), 5.13-5.09 (m, 1H), 4.94-4.92 (m, 1H), 4.55-4.45 (m, 2H), 4.19-4.15 (m, 1H), 3.18-3.17 (m, 2H); ${ }^{13} \mathrm{C}$ NMR (100 MHz, $\left.\mathrm{CDCl}_{3}\right): \delta 177.7,162.9\left(\mathrm{~d}, J_{\mathrm{F}-\mathrm{C}}=249.0 \mathrm{~Hz}\right), 143.8,136.2,134.0\left(\mathrm{~d}, J_{\mathrm{F}-\mathrm{C}}=9.0 \mathrm{~Hz}\right), 131.6,130.5,124.1$, $123.16,120.4,118.3\left(\mathrm{~d}, J_{\mathrm{F}-\mathrm{C}}=3.0 \mathrm{~Hz}\right), 115.8\left(\mathrm{~d}, J_{\mathrm{F}-\mathrm{C}}=22.0 \mathrm{~Hz}\right), 110.0,86.5,82.0,75.9,55.2,37.3$, 36.1; ${ }^{19} \mathrm{~F}$ NMR (376 MHz, $\mathrm{CDCl}_{3}$ ): $\delta$-110.05; IR (ATR): 2935, 1736, 1697, 1555, 1475, 1377, 1228 , $1174 \mathrm{~cm}^{-1}$; HRMS (ESI): Exact mass calcd for $\mathrm{C}_{21} \mathrm{H}_{19} \mathrm{~N}_{3} \mathrm{O}_{3} \mathrm{~F}^{35} \mathrm{Cl}_{2}\left[\mathrm{M}+\mathrm{NH}_{4}\right]^{+}$: 450.0782, Found: 450.0787 .

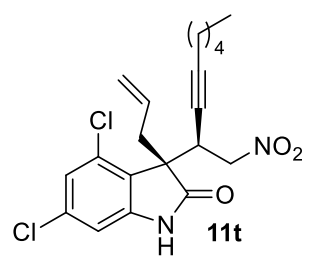

Product 11t was obtained in $93.8 \mathrm{mg}$ (92\% yield) as white solid. ${ }^{1} \mathrm{H}$ NMR analysis revealed that the dr value was 8:1. HPLC analysis: [Chiralcel IC, 5\% ${ }^{i} \mathrm{PrOH} /$ hexane, $1.0 \mathrm{~mL} / \mathrm{min}, 230 \mathrm{~nm}$; major diastereomer: $\mathrm{t}_{\mathrm{r}}(\operatorname{minor})=7.44 \mathrm{~min}, \mathrm{t}_{\mathrm{r}}$ $($ major $)=72.73$ min; minor diastereomer: $\mathrm{t}_{\mathrm{r}}($ minor $)=8.11$ min, $\mathrm{t}_{\mathrm{r}}($ major $)=$ $13.59 \mathrm{~min}]$ gave the isomeric composition of major diastereomer: $95 \%$ ee; $[\alpha]^{25} \mathrm{D}=-78.8(\mathrm{c}=0.21$, $\mathrm{CH}_{2} \mathrm{Cl}_{2}$ ). Mp 140-142 ${ }^{\circ} \mathrm{C} .{ }^{1} \mathrm{H}$ NMR (400 MHz, $\left.\mathrm{CDCl}_{3}\right): \delta$ 8.39-8.20 (m, $\left.1 \mathrm{H}\right)$, 7.05-7.05 (m, $\left.1 \mathrm{H}\right)$, 6.83-6.81 (m, 1H), 5.34-5.23 (m, 1H), 5.11-5.06 (m, 1H), 4.92-4.89 (m, 1H), 4.43-4.38 (m, 1H), 4.30-4.26 (m, 1H), 4.13-4.09 (m, 1H), 3.10-3.08 (m, 2H), 2.18 (td, $J=6.8,2.4 \mathrm{~Hz}, 2 \mathrm{H}), 1.52-1.45(\mathrm{~m}$, 2H), 1.37-1.26 (m, 4H), 0.90 (t, $J=7.2 \mathrm{~Hz}, 3 \mathrm{H}) ;{ }^{13} \mathrm{C} \mathrm{NMR}\left(100 \mathrm{MHz}, \mathrm{CDCl}_{3}\right): \delta 177.7,143.7,135.9$, 131.5, 130.6, 123.9, 123.4, 120.0, 109.6, 88.3, 76.4, 72.6, 55.1, 37.0, 35.8, 30.9, 28.2, 22.1, 18.7, 14.0; IR (ATR): 2929, 1731, 1692, 1580, 1434, 1335, 1206, $1092 \mathrm{~cm}^{-1}$; HRMS (ESI): Exact mass calcd for $\mathrm{C}_{20} \mathrm{H}_{26} \mathrm{~N}_{3} \mathrm{O}_{3}{ }^{35} \mathrm{Cl}_{2}\left[\mathrm{M}+\mathrm{NH}_{4}\right]^{+}:$426.1346, Found: 426.1353.

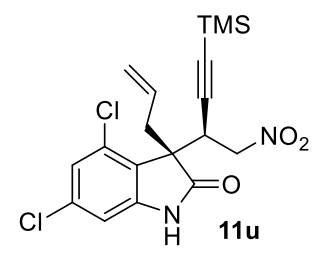

Product 11u was obtained in $87.1 \mathrm{mg}$ ( $85 \%$ yield) as white solid. ${ }^{1} \mathrm{H}$ NMR analysis revealed that the dr value was 10:1. HPLC analysis: [Chiralcel IC, 5\% ${ }^{i} \mathrm{PrOH} / \mathrm{hexane}, 1.0 \mathrm{~mL} / \mathrm{min}, 230 \mathrm{~nm}$; major diastereomer: $\mathrm{t}_{\mathrm{r}}($ minor $)=4.82 \mathrm{~min}, \mathrm{t}_{\mathrm{r}}$ $($ major $)=25.87 \mathrm{~min} ;$ minor diastereomer: $\mathrm{t}_{\mathrm{r}}($ minor $)=6.08 \mathrm{~min}, \mathrm{t}_{\mathrm{r}}($ major $)=11.38$ 
$\min ]$ gave the isomeric composition of major diastereomer: $91 \%$ ee; $[\alpha]^{25} \mathrm{D}=-52.4\left(\mathrm{c}=1.15, \mathrm{CH}_{2} \mathrm{Cl}_{2}\right)$. Mp 118-120 ${ }^{\circ} \mathrm{C} .{ }^{1} \mathrm{H}$ NMR (400 MHz, $\left.\mathrm{CDCl}_{3}\right): \delta 9.14(\mathrm{~s}, 1 \mathrm{H}), 7.05(\mathrm{~d}, J=1.6 \mathrm{~Hz}, 1 \mathrm{H}), 6.85(\mathrm{~d}, J=1.6$ $\mathrm{Hz}, 1 \mathrm{H}), 5.34-5.24(\mathrm{~m}, 1 \mathrm{H}), 5.11-5.07(\mathrm{~m}, 1 \mathrm{H}), 4.93-4.90(\mathrm{~m}, 1 \mathrm{H}), 4.47-4.42(\mathrm{~m}, 1 \mathrm{H}), 4.30(\mathrm{ABd}, J=$ 10.0, 4.0 Hz, 1H), $4.17(\mathrm{ABd}, J=12.0,4.0 \mathrm{~Hz}, 1 \mathrm{H}), 3.14-3.04(\mathrm{~m}, 2 \mathrm{H}), 0.16(\mathrm{~s}, 9 \mathrm{H}) ;{ }^{13} \mathrm{C} \mathrm{NMR}(100$ $\left.\mathrm{MHz}_{\mathrm{CDCl}}\right): \delta 177.5,143.9,136.1,131.6,130.6,124.0,123.4,120.3,109.8,98.4,93.1,76.0,55.1$, 37.0, 36.5, -0.2; IR (ATR): 1722, 1613, 1580, 1478, 1373, 1317, 1250, 1178, $1087 \mathrm{~cm}^{-1}$; HRMS (ESI): Exact mass calcd for $\mathrm{C}_{18} \mathrm{H}_{24} \mathrm{~N}_{3} \mathrm{O}_{3} \mathrm{Si}^{35} \mathrm{Cl}_{2}\left[\mathrm{M}+\mathrm{NH}_{4}\right]^{+}$: 428.0958 , Found: 428.0967.

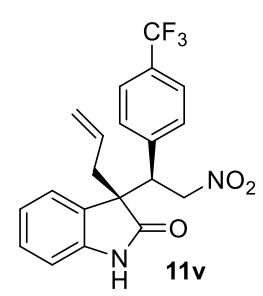

Product 11v was obtained in $68.3 \mathrm{mg}$ (70\% yield) as yellowish oil. ${ }^{1} \mathrm{H}$ NMR analysis revealed that the $\mathrm{dr}$ value was 6:1. HPLC analysis: [Chiralcel AD-H, 15\% ${ }^{i} \mathrm{PrOH} /$ hexane, $1.0 \mathrm{~mL} / \mathrm{min}, 254 \mathrm{~nm}$; major diastereomer: $\mathrm{t}_{\mathrm{r}}$ (major) $=7.07 \mathrm{~min}, \mathrm{t}_{\mathrm{r}}$ (minor) $=7.87 \mathrm{~min}]$ gave the isomeric composition of major diastereomer: $97 \%$ ee; $[\alpha]^{25} \mathrm{D}=-1.3\left(\mathrm{c}=0.50, \mathrm{CHCl}_{3}\right) .{ }^{1} \mathrm{H} \mathrm{NMR}\left(400 \mathrm{MHz}, \mathrm{CDCl}_{3}\right): \delta 7.73(\mathrm{~s}, 1 \mathrm{H})$, 7.42-7.40 (m, 2H), 7.31-7.27 (m, 1H), 7.18-7.11 (m, 4H), $6.79(\mathrm{~d}, J=8.0 \mathrm{~Hz}, 1 \mathrm{H}), 5.35-5.28(\mathrm{~m}, 1 \mathrm{H})$, 5.02-4.89 (m, 4H), 4.10 (dd, $J=9.2,2.4 \mathrm{~Hz}, 1 \mathrm{H}), 2.66-2.64(\mathrm{~m}, 2 \mathrm{H}) ;{ }^{13} \mathrm{C}$ NMR $\left(125 \mathrm{MHz}, \mathrm{CDCl}_{3}\right): \delta$ $177.8,140.8\left(\mathrm{~d}, J_{\mathrm{F}-\mathrm{C}}=2.5 \mathrm{~Hz}\right), 138.7,130.6,130.5\left(\mathrm{q}, J_{\mathrm{F}-\mathrm{C}}=32.5 \mathrm{~Hz}\right), 129.6,129.4,128.6,125.2(\mathrm{q}$, $\left.J_{\mathrm{F}-\mathrm{C}}=3.8 \mathrm{~Hz}\right), 124.0,123.9\left(\mathrm{q}, J_{\mathrm{F}-\mathrm{C}}=270.0 \mathrm{~Hz}\right), 123.0,120.2,110.1\left(\mathrm{~d}, J_{\mathrm{F}-\mathrm{C}}=2.5 \mathrm{~Hz}\right), 75.7,55.0$, 49.5, 39.4; ${ }^{19} \mathrm{~F}$ NMR (376 MHz, $\mathrm{CDCl}_{3}$ ): $\delta$-62.71; IR (ATR): 1716, 1560, 1473, 1327, 1169, 1119, 1071, 757, $739 \mathrm{~cm}^{-1}$; HRMS (ESI): Exact mass calcd for $\mathrm{C}_{20} \mathrm{H}_{17} \mathrm{~F}_{3} \mathrm{~N}_{2} \mathrm{NaO}_{3}[\mathrm{M}+\mathrm{Na}]^{+}$: 413.1083 , Found: 413.1081.

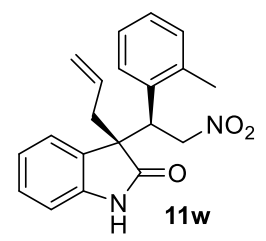

Product $11 \mathrm{w}$ was obtained in $61.3 \mathrm{mg}$ (73\% yield) as yellowish oil. ${ }^{1} \mathrm{H}$ NMR analysis revealed that the dr value was 6:1. HPLC analysis: [Chiralcel OD-H, 15\% ${ }^{i} \mathrm{PrOH} /$ hexane, $1.0 \mathrm{~mL} / \mathrm{min}, 254 \mathrm{~nm}$; major diastereomer: $\mathrm{t}_{\mathrm{r}}$ (major) $=6.72 \mathrm{~min}, \mathrm{t}_{\mathrm{r}}$ $($ minor $)=8.36 \mathrm{~min}]$ gave the isomeric composition of major diastereomer: $70 \%$ ee; $[\alpha]^{25} \mathrm{D}=-2.2\left(\mathrm{c}=0.50, \mathrm{CHCl}_{3}\right) .{ }^{1} \mathrm{H} \mathrm{NMR}\left(400 \mathrm{MHz}, \mathrm{CDCl}_{3}\right): \delta 7.91(\mathrm{~s}, 1 \mathrm{H}), 7.26-7.23(\mathrm{~m}, 1 \mathrm{H})$, 7.12-7.10 (m, 1H), 7.06-7.03 (m, 2H), 7.00-6.96 (m, 1H), 6.92-6.90 (m, 1H), $6.81(\mathrm{~d}, J=7.6 \mathrm{~Hz}, 1 \mathrm{H})$, $6.64(\mathrm{~d}, J=7.2 \mathrm{~Hz}, 1 \mathrm{H}), 5.28-5.22(\mathrm{~m}, 2 \mathrm{H}), 5.02-4.87(\mathrm{~m}, 3 \mathrm{H}), 4.31(\mathrm{dd}, J=11.6,4.0 \mathrm{~Hz}, 1 \mathrm{H})$, 2.74-2.72 (m, 2H), $2.11(\mathrm{t}, 3 \mathrm{H}) ;{ }^{13} \mathrm{C}$ NMR (125 MHz, $\left.\mathrm{CDCl}_{3}\right): \delta 179.1,140.9,138.7,133.8,131.1$, 131.0, 129.4, 128.9, 128.0, 126.5, 125.9, 124.4, 122.6, 119.8, 109.8, 76.3, 54.9, 43.0, 37.8, 19.8; IR (ATR): 1718, 1715, 1707, 1559, 1555, 749, 742, 685, $663 \mathrm{~cm}^{-1}$; HRMS (ESI): Exact mass calcd for $\mathrm{C}_{20} \mathrm{H}_{20} \mathrm{~N}_{2} \mathrm{NaO}_{3}[\mathrm{M}+\mathrm{Na}]^{+}:$359.1366, Found: 359.1360. 


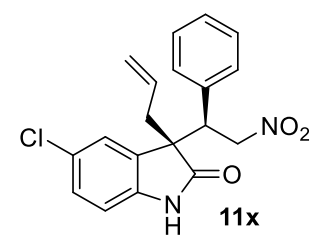

Product 11x was obtained in $70.3 \mathrm{mg}\left(79 \%\right.$ yield) as yellowish oil. ${ }^{1} \mathrm{H}$ NMR analysis revealed that the dr value was 9:1. HPLC analysis: [Chiralcel AD-H, 15\% ${ }^{i} \mathrm{PrOH} / \mathrm{h}$ exane, $1.0 \mathrm{~mL} / \mathrm{min}, 254 \mathrm{~nm}$; major diastereomer: $\mathrm{t}_{\mathrm{r}}$ (major) $=10.13 \mathrm{~min}$, $\left.\mathrm{t}_{\mathrm{r}}(\mathrm{minor})=9.08 \mathrm{~min}\right]$ gave the isomeric composition of major diastereomer: $97 \%$ ee; $[\alpha]^{25} \mathrm{D}=+13.6\left(\mathrm{c}=0.50, \mathrm{CH}_{2} \mathrm{Cl}_{2}\right) .{ }^{1} \mathrm{H} \mathrm{NMR}\left(400 \mathrm{MHz}, \mathrm{CDCl}_{3}\right): \delta 7.86(\mathrm{~s}, 1 \mathrm{H}), 7.26-7.18(\mathrm{~m}, 4 \mathrm{H})$, 7.02-6.95 (m, 3H), $6.72(\mathrm{~d}, J=8.4 \mathrm{~Hz}, 1 \mathrm{H}), 5.30-5.26(\mathrm{~m}, 1 \mathrm{H}), 5.03-4.90(\mathrm{~m}, 4 \mathrm{H}), 3.97(\mathrm{dd}, J=10.8$, $4.4 \mathrm{~Hz}, 1 \mathrm{H}), 2.67-2.63(\mathrm{~m}, 2 \mathrm{H}) ;{ }^{13} \mathrm{C} \mathrm{NMR}\left(125 \mathrm{MHz}, \mathrm{CDCl}_{3}\right): \delta 178.3,139.4,134.2,131.1,130.5$, 129.2, 129.0, 128.5, 128.4, 128.0, 124.7, 120.3, 110.9, 75.7, 55.6, 49.5, 38.7; IR (ATR): 1717, 1558, 1478, 1456, 1377, 1242, 912, 739, $708 \mathrm{~cm}^{-1}$; HRMS (ESI): Exact mass calcd for $\mathrm{C}_{19} \mathrm{H}_{17} \mathrm{~N}_{2} \mathrm{NaO}_{3} \mathrm{Cl}$ $[\mathrm{M}+\mathrm{Na}]^{+}: 379.0820$, Found: 379.0822 . 


\section{Product elaboration}

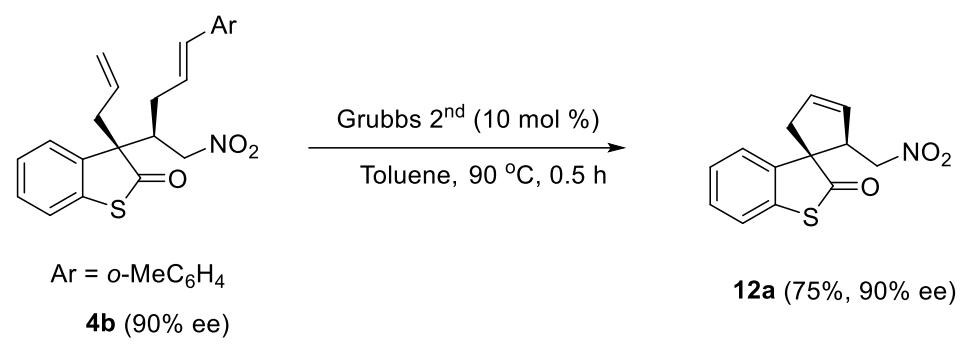

Under an atmosphere of nitrogen, to a Schlenk tube were added $4 \mathbf{b}$ (37.9 $\mathrm{mg}, 0.1 \mathrm{mmol}$ ), Grubbs $2^{\text {nd }}$ catalyst $(8.2 \mathrm{mg}, 0.01 \mathrm{mmol})$, and anhydrous toluene $(2.0 \mathrm{~mL})$. The resulting mixture was stirred at $90{ }^{\circ} \mathrm{C}$ about $0.5 \mathrm{~h}$ till full conversion of $\mathbf{4 b}$ by TLC analysis. The reaction mixture was directly subjected to column chromatography using EtOAc/PE (1:20, v/v) as eluent to afford the desired product 12a in $19.6 \mathrm{mg}(75 \%$ yield $)$ as colorless oil. $[\alpha]^{25} \mathrm{D}=-33.6\left(\mathrm{c}=0.50, \mathrm{CH}_{2} \mathrm{Cl}_{2}\right)$; $\mathrm{HPLC}$ analysis (Chiralcel OD-H, 1\% ${ }^{i} \mathrm{PrOH} /$ hexane, $1.0 \mathrm{~mL} / \mathrm{min}, 230 \mathrm{~nm}$; $\mathrm{t}_{\mathrm{r}}$ (major) $=23.75 \mathrm{~min}, \mathrm{t}_{\mathrm{r}}($ minor $)=20.31$ $\min )$ gave the isomeric composition of the product: $90 \%$ ee, ${ }^{1} \mathrm{H}$ NMR $\left(400 \mathrm{MHz}, \mathrm{CDCl}_{3}\right): \delta 7.36-7.26$ $(\mathrm{m}, 4 \mathrm{H}), 6.07-6.04(\mathrm{~m}, 1 \mathrm{H}), 5.75-5.72(\mathrm{~m}, 1 \mathrm{H}), 4.70,4.47(\mathrm{ABd}, J=14.4,8.0 \mathrm{~Hz}, 2 \mathrm{H}), 3.84-3.82(\mathrm{~m}$, $1 \mathrm{H}), 3.21-3.15(\mathrm{~m}, 1 \mathrm{H}), 2.79-2.74(\mathrm{~m}, 1 \mathrm{H}) ;{ }^{13} \mathrm{C} \mathrm{NMR}\left(100 \mathrm{MHz}, \mathrm{CDCl}_{3}\right): \delta 207.8,143.3,133.5$, 132.1, 128.8, 128.2, 127.3, 122.9, 74.5, 64.2, 55.8, 47.8; IR (ATR): 1705, 1551, 1466, 1377, 1339, 1111, 907, 826, $754 \mathrm{~cm}^{-1}$; HRMS (ESI): Exact mass calcd for $\mathrm{C}_{13} \mathrm{H}_{11} \mathrm{NNaO}_{3} \mathrm{~S}[\mathrm{M}+\mathrm{Na}]^{+}: 284.0352$, Found: 284.0346.

Compounds 12b-12d were obtained following a same procedure.

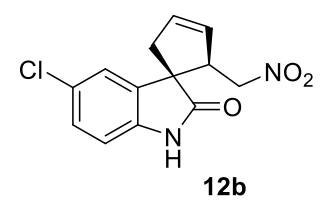

Product 12b was obtained in $21.1 \mathrm{mg}$ (76\% yield) as colorless oil. $[\alpha]^{25} \mathrm{D}=-20.7$ (c $=0.50, \mathrm{CH}_{2} \mathrm{Cl}_{2}$ ); HPLC analysis (Chiralcel AD-H, $15 \%{ }^{i} \mathrm{PrOH} /$ hexane, 1.0 $\mathrm{mL} / \mathrm{min}, 254 \mathrm{~nm} ; \mathrm{t}_{\mathrm{r}}$ (major) $=14.46 \mathrm{~min}, \mathrm{t}_{\mathrm{r}}($ minor $\left.)=10.55 \mathrm{~min}\right)$ gave the isomeric composition of the product: $97 \%$ ee; ${ }^{1} \mathrm{H}$ NMR $\left(400 \mathrm{MHz}, \mathrm{CDCl}_{3}\right): \delta$ 8.27 (s, 1H), 7.26-7.20 (m, 2H), 6.87-6.85 (m, 1H), 6.12-6.10 (m, 1H), 5.77-5.75 (m, 1H), 4.90, 4.43 $(\mathrm{ABd}, J=15.2,9.2 \mathrm{~Hz}, 2 \mathrm{H}), 3.76-3.74(\mathrm{~m}, 1 \mathrm{H}), 3.13-3.08(\mathrm{~m}, 1 \mathrm{H}), 2.70-2.65(\mathrm{~m}, 1 \mathrm{H}) ;{ }^{13} \mathrm{C} \mathrm{NMR}(125$ $\left.\mathrm{MHz}_{\mathrm{CDCl}}\right): \delta 180.0,138.0,137.9,133.0,128.3,128.4,128.2,122.3,111.0,73.4,53.6,52.6,44.4$; IR (ATR): 3259, 2927, 2858, 1712, 1611, 1580, 1467, 1376, $1347 \mathrm{~cm}^{-1}$; HRMS (ESI): Exact mass calcd for $\mathrm{C}_{13} \mathrm{H}_{11} \mathrm{~N}_{2} \mathrm{NaO}_{3} \mathrm{Cl}[\mathrm{M}+\mathrm{Na}]^{+}$: 301.0350, Found: 301.0359 . 


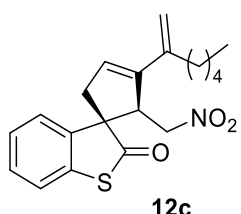

$12 \mathrm{c}$

Product 12c was obtained in $30.3 \mathrm{mg}(85 \%$ yield $)$ as colorless oil. $[\alpha]^{25} \mathrm{D}=+1.6(\mathrm{c}=$ $0.5, \mathrm{CH}_{2} \mathrm{Cl}_{2}$ ); HPLC analysis (Chiralcel AD-H, $1 \%{ }^{i} \mathrm{PrOH} / \mathrm{hexane}, 1.0 \mathrm{~mL} / \mathrm{min}, 230$ $\mathrm{nm} ; \mathrm{t}_{\mathrm{r}}($ major $)=10.53 \mathrm{~min}, \mathrm{t}_{\mathrm{r}}($ minor $\left.)=11.48 \mathrm{~min}\right)$ gave the isomeric composition of the product: $85 \%$ ee; ${ }^{1} \mathrm{H}$ NMR $\left(400 \mathrm{MHz}, \mathrm{CDCl}_{3}\right): \delta$ 7.37-7.21 (m, $\left.4 \mathrm{H}\right)$, 6.01-6.00 (m, 1H), 5.11-4.98 (m, 3H), 4.50 (ABd, $J=16.4,2.4 \mathrm{~Hz}, 1 \mathrm{H}), 4.00-3.97$ (m, 1H), 3.29-3.24 (m, 1H), $2.76(\mathrm{ABd}, J=17.6,3.2 \mathrm{~Hz}, 1 \mathrm{H}), 2.33-2.32(\mathrm{~m}, 2 \mathrm{H}), 1.57-1.52(\mathrm{~m}, 3 \mathrm{H}), 1.36-1.33(\mathrm{~m}, 3 \mathrm{H})$, 0.95-0.91 (m, 3H); ${ }^{13} \mathrm{C}$ NMR (100 MHz, $\left.\mathrm{CDCl}_{3}\right): \delta 208.0,144.0,141.9,140.5,133.2,128.8,127.2$, 127.0, 122.9, 122.2, 113.7, 71.5, 64.0, 54.5, 46.6, 34.5, 31.7, 28.2, 22.5, 14.1; IR (ATR): 1709, 1553, 1466, 1375, 1111, 1028, 900, 837, $758 \mathrm{~cm}^{-1}$; HRMS (ESI): Exact mass calcd for $\mathrm{C}_{20} \mathrm{H}_{23} \mathrm{NNaO}_{3} \mathrm{~S}$ $[\mathrm{M}+\mathrm{Na}]^{+}:$380.1291, Found: 380.1294 .

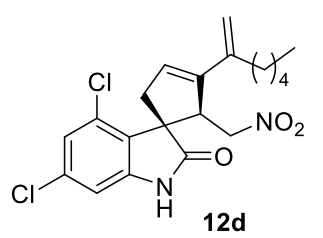

Product 12d was obtained in $31.4 \mathrm{mg}(77 \%$ yield $)$ as colorless oil. $[\alpha]^{25} \mathrm{D}=$ $-121.5\left(\mathrm{c}=1.22, \mathrm{CH}_{2} \mathrm{Cl}_{2}\right)$; HPLC analysis (Chiralcel IE, 5\% ${ }^{i} \mathrm{PrOH} / \mathrm{hexane}, 1.0$ $\mathrm{mL} / \mathrm{min}, 230 \mathrm{~nm} ; \mathrm{t}_{\mathrm{r}}($ major $)=14.99 \mathrm{~min}, \mathrm{t}_{\mathrm{r}}($ minor $\left.)=9.92 \mathrm{~min}\right)$ gave the isomeric composition of the product: $96 \%$ ee; ${ }^{1} \mathrm{H}$ NMR $\left(400 \mathrm{MHz}, \mathrm{CDCl}_{3}\right): \delta$ 8.49 (s, 1H), $7.03(\mathrm{~d}, J=1.2 \mathrm{~Hz}, 1 \mathrm{H}), 6.85(\mathrm{~d}, J=1.6 \mathrm{~Hz}, 1 \mathrm{H}), 5.94(\mathrm{~s}, 1 \mathrm{H}), 5.21-5.15(\mathrm{~m}, 1 \mathrm{H}), 5.07$ (s, 1H), $4.94(\mathrm{~s}, 1 \mathrm{H}), 4.74-4.69(\mathrm{~m}, 1 \mathrm{H}), 4.20(\mathrm{~d}, J=11.2 \mathrm{~Hz}, 1 \mathrm{H}), 3.17-3.12(\mathrm{~m}, 1 \mathrm{H}), 2.91-2.86(\mathrm{~m}$, $1 \mathrm{H}), 2.35-2.22(\mathrm{~m}, 2 \mathrm{H}), 1.55-1.42(\mathrm{~m}, 2 \mathrm{H}), 1.39-1.32(\mathrm{~m}, 4 \mathrm{H}), 0.92(\mathrm{t}, J=7.2 \mathrm{~Hz}, 3 \mathrm{H}) ;{ }^{13} \mathrm{C}$ NMR $\left(100 \mathrm{MHz}, \mathrm{CDCl}_{3}\right): \delta 180.44,142.47,142.39,140.24,134.67,131.43,130.63,127.51,123.82,112.85$, 109.50, 72.18, 54.08, 50.89, 43.60, 35.01, 31.78, 28.32, 22.63, 14.20; IR (ATR): 3259, 2927, 2858, 1712, 1611, 1580, 1467, 1376, $1347 \mathrm{~cm}^{-1}$; HRMS (ESI): Exact mass calcd for $\mathrm{C}_{20} \mathrm{H}_{26} \mathrm{~N}_{3} \mathrm{O}_{3}{ }^{35} \mathrm{Cl}_{2}$ $\left[\mathrm{M}+\mathrm{NH}_{4}\right]^{+}:$426.1346, Found: 426.1349 .
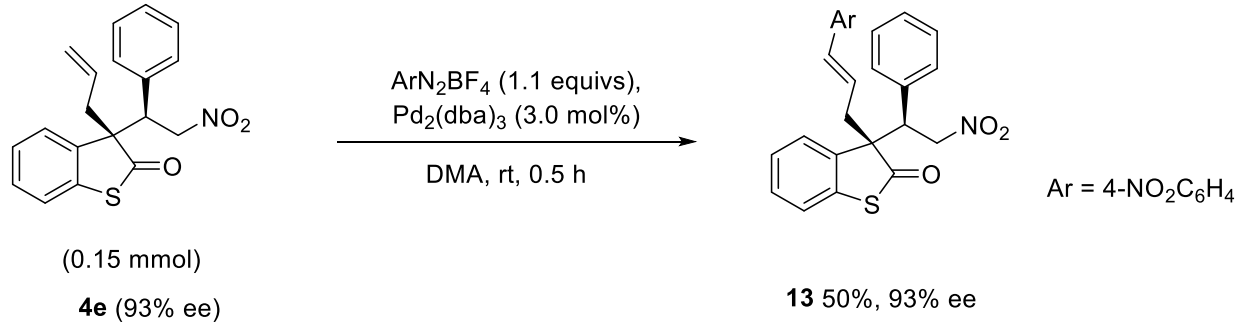

4e $(93 \%$ ee $)$

$1350 \%, 93 \%$ ee

Under an atmosphere of nitrogen, to a Schlenk tube were added $\operatorname{Pd}_{2}(\mathrm{dba})_{3}(4.1 \mathrm{mg}, 0.0045 \mathrm{mmol})$, 4e $(50.9 \mathrm{mg}, 0.15 \mathrm{mmol})$, benzenediazonium salt ${ }^{10}(31.7 \mathrm{mg}, 0.17 \mathrm{mmol})$ and $2.5 \mathrm{~mL}$ DMA. The resulting mixture was stirred at room temperature for $0.5 \mathrm{~h}$ till full conversion of $4 \mathrm{e}$. Then $10 \mathrm{~mL} \mathrm{Et}_{2} \mathrm{O}$ 
and $10 \mathrm{~mL}$ of water was added, and the aqueous layer was extracted twice with $10 \mathrm{~mL} \mathrm{Et} 2 \mathrm{O}$. The combined organic layers were dried over $\mathrm{Na}_{2} \mathrm{SO}_{4}$. After concentration in vacuo, the residue was purified by silica gel column chromatography using EtOAc/PE (1:5, v/v) as eluent to afford the desired product 13 in $33.8 \mathrm{mg}$ (50\% yield) as yellow solid. Mp 143-145 ${ }^{\circ} \mathrm{C} .[\alpha]^{25} \mathrm{D}=+9.9(\mathrm{c}=0.50$, $\mathrm{CH}_{2} \mathrm{Cl}_{2}$ ); HPLC analysis (Chiralcel AD-H, 40\% ${ }^{i} \mathrm{PrOH} / \mathrm{hexane}, 1.0 \mathrm{~mL} / \mathrm{min}, 230 \mathrm{~nm}$; $\mathrm{t}_{\mathrm{r}}$ (major) = $22.98 \mathrm{~min}, \mathrm{t}_{\mathrm{r}}($ minor $\left.)=13.02 \mathrm{~min}\right)$ gave the isomeric composition of the product: $93 \%$ ee; ${ }^{1} \mathrm{H} \mathrm{NMR}$ $\left(400 \mathrm{MHz}, \mathrm{CDCl}_{3}\right): \delta 8.06-8.04(\mathrm{~m}, 2 \mathrm{H}), 7.41-7.39(\mathrm{~m}, 2 \mathrm{H}), 7.32-7.30(\mathrm{~m}, 3 \mathrm{H}), 7.25-7.19(\mathrm{~m}, 4 \mathrm{H})$, 6.99-6.96 (m, 2H), 6.36-6.32 (m, 1H), 5.83-5.77 (m, 1H), 4.97-4.91 (m, 1H), 4.78 (ABd, $J=12.8,4.4$ $\mathrm{Hz}, 1 \mathrm{H}), 4.08(\mathrm{dd}, J=11.2,4.4 \mathrm{~Hz}, 1 \mathrm{H}), 2.88-2.86(\mathrm{~m}, 2 \mathrm{H}), 2.70-2.65(\mathrm{~m}, 1 \mathrm{H}) ;{ }^{13} \mathrm{C} \mathrm{NMR}(125 \mathrm{MHz}$, $\left.\mathrm{CDCl}_{3}\right): \delta 206.3,147.0,142.9,136.5,136.2,133.4,133.3,129.7,129.4,128.7,128.5,126.9,126.8$, 124.8, 124.0, 123.9, 123.6, 75.5, 65.2, 52.2, 40.5; IR (ATR): 1703, 1557, 1516, 1377, 1342, 1109, 862, 746, $702 \mathrm{~cm}^{-1}$; HRMS (ESI): Exact mass calcd for $\mathrm{C}_{25} \mathrm{H}_{20} \mathrm{~N}_{2} \mathrm{NaO}_{5} \mathrm{~S}[\mathrm{M}+\mathrm{Na}]^{+}$: 483.0985, Found: 483.0993. 


\section{X-ray crystallographic data of $11 \mathrm{r}$ and 13.}

The solvent system of $n$-hexane/EtOAc (4/1, v/v) was employed for crystal growth. The ellipsoid contour 50\% probability levels in the caption for the image of the structure.

Data intensity of $\mathbf{1 1} \mathbf{r}^{1}$ was collected using a 'Bruker APEX-II CCD' diffractometer at 296(2) K. Data collection and reduction were done by using Olex2 and the structure was solved with the ShelXS structure solution program using direct methods and refined by full-matrix least-squares on $F^{2}$ with anisotropic displacement parameters for non-H atoms using SHELX-97. Hydrogen atoms were added at their geometrically idea positions and refined isotropically. Crystal data for $11 \mathrm{r}$ : $\mathrm{C}_{22} \mathrm{H}_{18} \mathrm{Cl}_{2} \mathrm{~N}_{2} \mathrm{O}_{4}, \mathrm{M}$ $=445.28, T=293(2) \mathrm{K}, \lambda=0.71073 \AA$, Monoclinic, space group $\mathrm{P} 21 / \mathrm{c}, a=10.1586(10) \AA, b=$ 8.5870(8) $\AA, c=13.0464(12) \AA, V=1068.49(18) \AA^{3}, Z=2, d_{\text {calc }}=1.384 \mathrm{Mg} / \mathrm{m}^{3} .6512$ reflections measured, $4202[\mathrm{R}($ int $)=0.0203], \mathrm{R}_{1}=0.0416, \mathrm{wR}_{2}=0.1034\left(I>2 \sigma(I)\right.$, final $\mathrm{R}_{1}=0.0385, \mathrm{wR}_{2}=$ $0.1015, \mathrm{GOF}=1.071$, and 285 parameters.

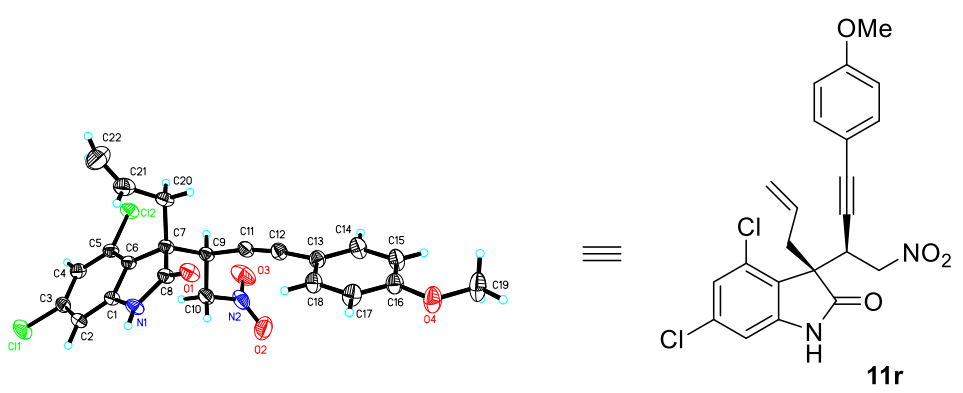

Table S7. Crystal data and structure refinement for 11r.

Identification code

Empirical formula

Formula weight

Temperature

Wavelength

Crystal system

Space group

Unit cell dimensions 11r

$\mathrm{C}_{22} \mathrm{H}_{18} \mathrm{Cl}_{2} \mathrm{~N}_{2} \mathrm{O}_{4}$

445.28

293(2) K

$0.71073 \AA$

Monoclinic

P 21

$a=10.1586(10) \AA \quad \alpha=90^{\circ}$.

$\mathrm{b}=8.5870(8) \AA \quad \beta=110.138(2)^{\circ}$.

${ }^{1}$ Supplementary crystallographic data have been deposited at the Cambridge Crystallographic Data Center. (CCDC1024589). 
Volume

$\mathrm{Z}$

Density (calculated)

Absorption coefficient

$\mathrm{F}(000)$

Crystal size

Theta range for data collection

Index ranges

Reflections collected

Independent reflections

Completeness to theta $=25.242^{\circ}$

Absorption correction

Max. and min. transmission

Refinement method

Data / restraints / parameters

Goodness-of-fit on $\mathrm{F}^{2}$

Final R indices [I $>2 \operatorname{sigma}(\mathrm{I})]$

$\mathrm{R}$ indices (all data)

Absolute structure parameter

Extinction coefficient

Largest diff. peak and hole $\mathrm{c}=13.0464(12) \AA \quad \gamma=90^{\circ}$.

1068.49(18) $\AA^{3}$

2

$1.384 \mathrm{Mg} / \mathrm{m}^{3}$

$0.335 \mathrm{~mm}^{-1}$

460

$0.211 \times 0.165 \times 0.101 \mathrm{~mm}^{3}$

1.663 to $25.996^{\circ}$

$-12<=\mathrm{h}<=6,-10<=\mathrm{k}<=10,-15<=1<=16$

6512

$4202[\mathrm{R}(\mathrm{int})=0.0203]$

$100.0 \%$

Semi-empirical from equivalents

0.7457 and 0.6670

Full-matrix least-squares on $\mathrm{F}^{2}$

4202 / 9 / 285

1.071

$\mathrm{R} 1=0.0385, \mathrm{wR} 2=0.1015$

$\mathrm{R} 1=0.0416, \mathrm{wR} 2=0.1034$

$0.01(3)$

$\mathrm{n} / \mathrm{a}$

0.239 and -0.185 e. $\AA^{-3}$

Table S8. Fractional Atomic Coordinates $\left(\times 10^{4}\right)$ and Equivalent Isotropic Displacement Parameters $\left(\AA^{2} \times 10^{3}\right)$ for 11r. $U_{\text {eq }}$ is defined as $1 / 3$ of of the trace of the orthogonalised $U_{\text {IJ }}$ tensor.

\begin{tabular}{ccccc}
\hline Atom & $\boldsymbol{x}$ & $\boldsymbol{y}$ & $\boldsymbol{z}$ & $\mathbf{U}(\mathbf{e q})$ \\
\hline $\mathrm{Cl}(1)$ & $995(1)$ & $-2048(1)$ & $5943(1)$ & $72(1)$ \\
$\mathrm{Cl}(2)$ & $2290(1)$ & $3996(1)$ & $6551(1)$ & $55(1)$ \\
$\mathrm{N}(1)$ & $631(3)$ & $1136(3)$ & $9189(2)$ & $44(1)$ \\
$\mathrm{N}(2)$ & $5434(3)$ & $3105(4)$ & $10247(3)$ & $67(1)$
\end{tabular}




\begin{tabular}{|c|c|c|c|c|}
\hline $\mathrm{O}(1)$ & $467(3)$ & $3197(3)$ & $10246(2)$ & $51(1)$ \\
\hline $\mathrm{O}(2)$ & $6073(4)$ & $2569(5)$ & $11145(3)$ & $97(1)$ \\
\hline $\mathrm{O}(3)$ & $5898(3)$ & 3982(4) & $9728(4)$ & $96(1)$ \\
\hline $\mathrm{O}(4)$ & $3758(3)$ & $6428(4)$ & $15594(2)$ & $71(1)$ \\
\hline $\mathrm{C}(1)$ & $939(3)$ & $901(3)$ & $8235(2)$ & $37(1)$ \\
\hline $\mathrm{C}(2)$ & $839(3)$ & $-459(4)$ & $7667(3)$ & $42(1)$ \\
\hline $\mathrm{C}(3)$ & $1157(4)$ & $-377(4)$ & $6723(3)$ & $46(1)$ \\
\hline $\mathrm{C}(4)$ & $1579(4)$ & 971(4) & $6364(3)$ & $45(1)$ \\
\hline$C(5)$ & 1701(3) & $2308(4)$ & $6984(2)$ & $40(1)$ \\
\hline $\mathrm{C}(6)$ & $1368(3)$ & $2308(3)$ & $7922(2)$ & $36(1)$ \\
\hline $\mathrm{C}(7)$ & $1421(3)$ & $3533(3)$ & $8758(2)$ & $38(1)$ \\
\hline $\mathrm{C}(8)$ & $785(3)$ & $2647(3)$ & $9506(3)$ & $40(1)$ \\
\hline $\mathrm{C}(9)$ & $2975(3)$ & $4010(4)$ & $9399(2)$ & $41(1)$ \\
\hline$C(10)$ & $3948(4)$ & 2593(4) & $9706(3)$ & $54(1)$ \\
\hline $\mathrm{C}(11)$ & $3113(4)$ & $4893(4)$ & $10395(3)$ & $45(1)$ \\
\hline $\mathrm{C}(12)$ & $3309(4)$ & $5407(4)$ & 11279(3) & $47(1)$ \\
\hline$C(13)$ & $3424(4)$ & $5815(4)$ & $12370(3)$ & $46(1)$ \\
\hline $\mathrm{C}(14)$ & $4357(4)$ & $6906(5)$ & 12991(3) & $57(1)$ \\
\hline$C(15)$ & $4504(4)$ & $7148(5)$ & $14076(3)$ & $58(1)$ \\
\hline$C(16)$ & $3687(4)$ & $6321(5)$ & $14536(3)$ & $52(1)$ \\
\hline $\mathrm{C}(17)$ & $2726(4)$ & $5249(5)$ & 13918(3) & $56(1)$ \\
\hline $\mathrm{C}(18)$ & $2604(4)$ & $5001(5)$ & $12854(3)$ & $53(1)$ \\
\hline C(19) & $4657(6)$ & $7563(7)$ & $16268(3)$ & $85(2)$ \\
\hline$C(20)$ & 553(4) & $5012(4)$ & $8314(3)$ & $47(1)$ \\
\hline $\mathrm{C}(21)$ & $-939(5)$ & $4681(5)$ & $7655(4)$ & $75(1)$ \\
\hline $\mathrm{C}(22)$ & $-1571(11)$ & $5118(15)$ & $6703(8)$ & $110(3)$ \\
\hline$C\left(22^{\prime}\right)$ & $-2024(12)$ & $5262(18)$ & $7749(13)$ & $93(4)$ \\
\hline
\end{tabular}

Table S9. Anisotropic Displacement Parameters $\left(\AA^{2} \times 10^{3}\right)$ for 11r. The Anisotropic displacement factor exponent takes the form: $-2 \pi^{2}\left[h^{2} a^{* 2} U_{11}+2 h k a * b * U_{12}+\ldots\right]$. 


\begin{tabular}{|c|c|c|c|c|c|c|}
\hline Atom & $\mathbf{U}_{11}$ & $\mathbf{U}_{22}$ & $\mathbf{U}_{33}$ & $\mathbf{U}_{23}$ & $\mathbf{U}_{13}$ & $\mathbf{U}_{12}$ \\
\hline $\mathrm{Cl}(1)$ & 103(1) & $46(1)$ & $72(1)$ & $-23(1)$ & $36(1)$ & $-1(1)$ \\
\hline $\mathrm{Cl}(2)$ & $82(1)$ & $44(1)$ & $52(1)$ & $7(1)$ & $39(1)$ & $-5(1)$ \\
\hline $\mathrm{N}(1)$ & $62(2)$ & $33(1)$ & $49(2)$ & $1(1)$ & $34(1)$ & $-5(1)$ \\
\hline $\mathrm{N}(2)$ & $58(2)$ & $50(2)$ & $89(3)$ & $-22(2)$ & $20(2)$ & $1(2)$ \\
\hline $\mathrm{O}(1)$ & $72(2)$ & $43(1)$ & $54(1)$ & $-4(1)$ & $41(1)$ & $0(1)$ \\
\hline $\mathrm{O}(2)$ & $83(2)$ & 109(3) & $74(2)$ & $-17(2)$ & $-4(2)$ & $10(2)$ \\
\hline $\mathrm{O}(3)$ & $72(2)$ & $61(2)$ & 163(4) & $-4(2)$ & $50(2)$ & $-5(2)$ \\
\hline $\mathrm{O}(4)$ & $86(2)$ & $88(2)$ & $47(1)$ & $-17(1)$ & $33(1)$ & $-25(2)$ \\
\hline $\mathrm{C}(1)$ & $41(1)$ & $33(1)$ & $39(1)$ & $2(1)$ & $17(1)$ & $2(1)$ \\
\hline $\mathrm{C}(2)$ & $47(2)$ & $30(1)$ & $50(2)$ & $-1(1)$ & $19(1)$ & $-1(1)$ \\
\hline $\mathrm{C}(3)$ & $51(2)$ & $37(2)$ & $48(2)$ & $-10(1)$ & $14(1)$ & $6(1)$ \\
\hline $\mathrm{C}(4)$ & $56(2)$ & $46(2)$ & $36(2)$ & $0(1)$ & $20(1)$ & $8(1)$ \\
\hline$C(5)$ & $48(2)$ & $36(1)$ & $40(2)$ & $6(1)$ & $19(1)$ & $2(1)$ \\
\hline$C(6)$ & $41(2)$ & $32(1)$ & $36(1)$ & $3(1)$ & $16(1)$ & $2(1)$ \\
\hline$C(7)$ & $49(2)$ & $32(1)$ & $38(1)$ & $0(1)$ & $21(1)$ & $0(1)$ \\
\hline $\mathrm{C}(8)$ & $48(2)$ & $34(2)$ & $43(2)$ & $2(1)$ & $21(1)$ & $0(1)$ \\
\hline $\mathrm{C}(9)$ & $54(2)$ & $32(1)$ & $40(1)$ & $0(1)$ & $22(1)$ & $-2(1)$ \\
\hline$C(10)$ & $56(2)$ & $40(2)$ & $61(2)$ & $-7(2)$ & $16(2)$ & $0(2)$ \\
\hline $\mathrm{C}(11)$ & $55(2)$ & $37(2)$ & $47(2)$ & $-3(1)$ & $22(1)$ & $-4(1)$ \\
\hline$C(12)$ & $54(2)$ & $44(2)$ & $48(2)$ & $-4(1)$ & $22(2)$ & $-4(2)$ \\
\hline$C(13)$ & $50(2)$ & $48(2)$ & $41(2)$ & $-4(1)$ & $19(1)$ & $2(2)$ \\
\hline$C(14)$ & $68(2)$ & $59(2)$ & $56(2)$ & $-13(2)$ & $35(2)$ & $-19(2)$ \\
\hline$C(15)$ & $65(2)$ & $58(2)$ & $52(2)$ & $-17(2)$ & $24(2)$ & $-18(2)$ \\
\hline$C(16)$ & $55(2)$ & $60(2)$ & $45(2)$ & $-7(2)$ & $23(2)$ & $-5(2)$ \\
\hline$C(17)$ & $52(2)$ & $72(2)$ & $50(2)$ & $-3(2)$ & $24(2)$ & $-11(2)$ \\
\hline$C(18)$ & $47(2)$ & $60(2)$ & $50(2)$ & $-8(2)$ & $15(2)$ & $-9(2)$ \\
\hline C(19) & $97(3)$ & $106(4)$ & $51(2)$ & $-31(2)$ & $26(2)$ & $-26(3)$ \\
\hline$C(20)$ & $63(2)$ & $33(2)$ & $52(2)$ & $4(1)$ & $28(2)$ & $7(1)$ \\
\hline$C(21)$ & $69(3)$ & $53(2)$ & $93(3)$ & $7(2)$ & $16(2)$ & $11(2)$ \\
\hline
\end{tabular}




\begin{tabular}{ccccccc} 
C(22) & $91(6)$ & $115(7)$ & $96(6)$ & $21(6)$ & $-1(5)$ & $6(6)$ \\
C(22') & $64(6)$ & $92(8)$ & $105(8)$ & $2(7)$ & $7(6)$ & $15(6)$ \\
\hline
\end{tabular}

Table S10. Bond Lengths for 11r.

\begin{tabular}{|c|c|c|c|c|c|}
\hline Atom & Atom & Length/Å & Atom & Atom & Length/Å \\
\hline $\mathrm{Cl}(1)$ & $\mathrm{C}(3)$ & $1.734(3)$ & $\mathrm{C}(11)$ & $\mathrm{C}(12)$ & $1.185(5)$ \\
\hline $\mathrm{Cl}(2)$ & $\mathrm{C}(5)$ & $1.735(3)$ & $\mathrm{C}(12)$ & $C(13)$ & $1.432(4)$ \\
\hline $\mathrm{N}(1)$ & $\mathrm{C}(8)$ & $1.355(4)$ & $\mathrm{C}(13)$ & $C(14)$ & $1.380(5)$ \\
\hline $\mathrm{N}(1)$ & $\mathrm{C}(1)$ & $1.399(4)$ & $\mathrm{C}(13)$ & $\mathrm{C}(18)$ & $1.393(5)$ \\
\hline $\mathrm{N}(1)$ & $\mathrm{H}(1)$ & $0.81(5)$ & $\mathrm{C}(14)$ & $C(15)$ & $1.387(5)$ \\
\hline $\mathrm{N}(2)$ & $\mathrm{O}(3)$ & $1.211(5)$ & $\mathrm{C}(14)$ & $\mathrm{H}(14)$ & 0.9300 \\
\hline $\mathrm{N}(2)$ & $\mathrm{O}(2)$ & $1.218(5)$ & $\mathrm{C}(15)$ & $C(16)$ & $1.377(5)$ \\
\hline $\mathrm{N}(2)$ & $C(10)$ & $1.496(5)$ & $\mathrm{C}(15)$ & $\mathrm{H}(15)$ & 0.9300 \\
\hline $\mathrm{O}(1)$ & $\mathrm{C}(8)$ & $1.215(4)$ & $C(16)$ & $\mathrm{C}(17)$ & $1.381(5)$ \\
\hline $\mathrm{O}(4)$ & $C(16)$ & $1.360(4)$ & $\mathrm{C}(17)$ & $\mathrm{C}(18)$ & $1.368(5)$ \\
\hline $\mathrm{O}(4)$ & $C(19)$ & $1.416(6)$ & $\mathrm{C}(17)$ & $\mathrm{H}(17)$ & 0.9300 \\
\hline $\mathrm{C}(1)$ & $\mathrm{C}(2)$ & $1.368(4)$ & $\mathrm{C}(18)$ & $\mathrm{H}(18)$ & 0.9300 \\
\hline $\mathrm{C}(1)$ & $C(6)$ & $1.392(4)$ & $\mathrm{C}(19)$ & $\mathrm{H}(19 \mathrm{~A})$ & 0.9600 \\
\hline $\mathrm{C}(2)$ & $\mathrm{C}(3)$ & $1.379(5)$ & $\mathrm{C}(19)$ & $\mathrm{H}(19 \mathrm{~B})$ & 0.9600 \\
\hline $\mathrm{C}(2)$ & $\mathrm{H}(2 \mathrm{~A})$ & 0.9300 & $\mathrm{C}(19)$ & $\mathrm{H}(19 \mathrm{C})$ & 0.9600 \\
\hline $\mathrm{C}(3)$ & $\mathrm{C}(4)$ & $1.371(5)$ & $\mathrm{C}(20)$ & $\mathrm{C}(21)$ & $1.490(6)$ \\
\hline $\mathrm{C}(4)$ & $\mathrm{C}(5)$ & $1.385(4)$ & $\mathrm{C}(20)$ & $\mathrm{H}(20 \mathrm{~A})$ & 0.9700 \\
\hline $\mathrm{C}(4)$ & $\mathrm{H}(4)$ & 0.9300 & $\mathrm{C}(20)$ & $\mathrm{H}(20 \mathrm{~B})$ & 0.9700 \\
\hline $\mathrm{C}(5)$ & $C(6)$ & $1.378(4)$ & $\mathrm{C}(21)$ & $\mathrm{C}(22)$ & $1.243(10)$ \\
\hline $\mathrm{C}(6)$ & $\mathrm{C}(7)$ & $1.502(4)$ & $\mathrm{C}(21)$ & $C\left(22^{\prime}\right)$ & $1.254(13)$ \\
\hline $\mathrm{C}(7)$ & $C(20)$ & $1.541(4)$ & $\mathrm{C}(21)$ & $\mathrm{H}(21)$ & 0.9524 \\
\hline $\mathrm{C}(7)$ & $\mathrm{C}(8)$ & $1.543(4)$ & $\mathrm{C}(21)$ & $\mathrm{H}\left(21^{\prime}\right)$ & 0.9664 \\
\hline $\mathrm{C}(7)$ & $\mathrm{C}(9)$ & $1.565(4)$ & $\mathrm{C}(22)$ & $\mathrm{H}(22 \mathrm{~A})$ & 0.9300 \\
\hline $\mathrm{C}(9)$ & $\mathrm{C}(11)$ & $1.469(4)$ & $\mathrm{C}(22)$ & $\mathrm{H}(22 \mathrm{~B})$ & 0.9300 \\
\hline $\mathrm{C}(9)$ & $C(10)$ & $1.532(5)$ & $\mathrm{C}\left(22^{\prime}\right)$ & $\mathrm{H}(22 \mathrm{C})$ & 0.9300 \\
\hline $\mathrm{C}(9)$ & $\mathrm{H}(9)$ & 0.9800 & $\mathrm{C}\left(22^{\prime}\right)$ & $\mathrm{H}(22 \mathrm{D})$ & 0.9300 \\
\hline
\end{tabular}


$\mathrm{C}(10) \quad \mathrm{H}(10 \mathrm{~A}) \quad 0.9700$

$\begin{array}{lll}\mathrm{C}(10) & \mathrm{H}(10 \mathrm{~B}) & 0.9700\end{array}$

Table S11. Bond Angles for 11r.

\begin{tabular}{cccc|cccc}
\hline Atom & Atom & Atom & Angle/ & Atom & Atom & Atom & Angle/ \\
\hline $\mathrm{C}(8)$ & $\mathrm{N}(1)$ & $\mathrm{C}(1)$ & $111.8(3)$ & $\mathrm{N}(2)$ & $\mathrm{C}(10)$ & $\mathrm{H}(10 \mathrm{~A})$ & 109.6 \\
$\mathrm{C}(8)$ & $\mathrm{N}(1)$ & $\mathrm{H}(1)$ & $122(3)$ & $\mathrm{C}(9)$ & $\mathrm{C}(10)$ & $\mathrm{H}(10 \mathrm{~A})$ & 109.6 \\
$\mathrm{C}(1)$ & $\mathrm{N}(1)$ & $\mathrm{H}(1)$ & $125(3)$ & $\mathrm{N}(2)$ & $\mathrm{C}(10)$ & $\mathrm{H}(10 \mathrm{~B})$ & 109.6 \\
$\mathrm{O}(3)$ & $\mathrm{N}(2)$ & $\mathrm{O}(2)$ & $126.3(4)$ & $\mathrm{C}(9)$ & $\mathrm{C}(10)$ & $\mathrm{H}(10 \mathrm{~B})$ & 109.6 \\
$\mathrm{O}(3)$ & $\mathrm{N}(2)$ & $\mathrm{C}(10)$ & $116.3(4)$ & $\mathrm{H}(10 \mathrm{~A})$ & $\mathrm{C}(10)$ & $\mathrm{H}(10 \mathrm{~B})$ & 108.1 \\
$\mathrm{O}(2)$ & $\mathrm{N}(2)$ & $\mathrm{C}(10)$ & $117.4(4)$ & $\mathrm{C}(12)$ & $\mathrm{C}(11)$ & $\mathrm{C}(9)$ & $170.2(4)$ \\
$\mathrm{C}(16)$ & $\mathrm{O}(4)$ & $\mathrm{C}(19)$ & $118.3(3)$ & $\mathrm{C}(11)$ & $\mathrm{C}(12)$ & $\mathrm{C}(13)$ & $170.8(4)$ \\
$\mathrm{C}(2)$ & $\mathrm{C}(1)$ & $\mathrm{C}(6)$ & $123.7(3)$ & $\mathrm{C}(14)$ & $\mathrm{C}(13)$ & $\mathrm{C}(18)$ & $118.2(3)$ \\
$\mathrm{C}(2)$ & $\mathrm{C}(3)$ & $\mathrm{Cl}(1)$ & $118.5(3)$ & $\mathrm{C}(14)$ & $\mathrm{C}(13)$ & $\mathrm{C}(12)$ & $123.6(3)$ \\
$\mathrm{C}(3)$ & $\mathrm{C}(4)$ & $\mathrm{C}(5)$ & $118.5(3)$ & $\mathrm{C}(18)$ & $\mathrm{C}(13)$ & $\mathrm{C}(12)$ & $118.1(3)$ \\
$\mathrm{C}(3)$ & $\mathrm{C}(4)$ & $\mathrm{H}(4)$ & 120.8 & $\mathrm{C}(13)$ & $\mathrm{C}(14)$ & $\mathrm{C}(15)$ & $120.7(3)$ \\
$\mathrm{C}(5)$ & $\mathrm{C}(4)$ & $\mathrm{H}(4)$ & 120.8 & $\mathrm{C}(13)$ & $\mathrm{C}(14)$ & $\mathrm{H}(14)$ & 119.6 \\
$\mathrm{C}(6)$ & $\mathrm{C}(5)$ & $\mathrm{C}(4)$ & $121.2(3)$ & $\mathrm{C}(15)$ & $\mathrm{C}(14)$ & $\mathrm{H}(14)$ & 119.6 \\
$\mathrm{C}(6)$ & $\mathrm{C}(5)$ & $\mathrm{Cl}(2)$ & $120.5(2)$ & $\mathrm{C}(16)$ & $\mathrm{C}(15)$ & $\mathrm{C}(14)$ & $119.9(3)$ \\
$\mathrm{C}(4)$ & $\mathrm{C}(5)$ & $\mathrm{Cl}(2)$ & $118.3(2)$ & $\mathrm{C}(16)$ & $\mathrm{C}(15)$ & $\mathrm{H}(15)$ & 120.0 \\
$\mathrm{C}(5)$ & $\mathrm{C}(6)$ & $\mathrm{C}(1)$ & $117.3(3)$ & $\mathrm{C}(14)$ & $\mathrm{C}(15)$ & $\mathrm{H}(15)$ & 120.0 \\
$\mathrm{C}(5)$ & $\mathrm{C}(6)$ & $\mathrm{C}(7)$ & $133.3(3)$ & $\mathrm{O}(4)$ & $\mathrm{C}(16)$ & $\mathrm{C}(15)$ & $125.3(3)$ \\
$\mathrm{C}(1)$ & $\mathrm{C}(6)$ & $\mathrm{C}(7)$ & $109.3(2)$ & $\mathrm{O}(4)$ & $\mathrm{C}(16)$ & $\mathrm{C}(17)$ & $114.6(3)$ \\
$\mathrm{C}(6)$ & $\mathrm{C}(7)$ & $\mathrm{C}(20)$ & $115.5(2)$ & $\mathrm{C}(15)$ & $\mathrm{C}(16)$ & $\mathrm{C}(17)$ & $120.0(3)$ \\
$\mathrm{C}(6)$ & $\mathrm{C}(7)$ & $\mathrm{C}(8)$ & $101.3(2)$ & $\mathrm{C}(18)$ & $\mathrm{C}(17)$ & $\mathrm{C}(16)$ & $119.6(3)$ \\
$\mathrm{C}(20)$ & $\mathrm{C}(7)$ & $\mathrm{C}(8)$ & $109.5(2)$ & $\mathrm{C}(18)$ & $\mathrm{C}(17)$ & $\mathrm{H}(17)$ & 120.2 \\
$\mathrm{C}(6)$ & $\mathrm{C}(7)$ & $\mathrm{C}(9)$ & $110.4(2)$ & $\mathrm{C}(16)$ & $\mathrm{C}(17)$ & $\mathrm{H}(17)$ & 120.2 \\
$\mathrm{C}(20)$ & $\mathrm{C}(7)$ & $\mathrm{C}(9)$ & $109.0(3)$ & $\mathrm{C}(17)$ & $\mathrm{C}(18)$ & $\mathrm{C}(13)$ & $121.5(3)$ \\
$\mathrm{C}(2)$ & $\mathrm{C}(3)$ & $\mathrm{Cl}(1)$ & $118.5(3)$ & $\mathrm{C}(17)$ & $\mathrm{C}(18)$ & $\mathrm{H}(18)$ & 119.3 \\
$\mathrm{C}(3)$ & $\mathrm{C}(4)$ & $\mathrm{C}(5)$ & $118.5(3)$ & $\mathrm{C}(13)$ & $\mathrm{C}(18)$ & $\mathrm{H}(18)$ & 119.3
\end{tabular}




\begin{tabular}{|c|c|c|c|c|c|c|c|}
\hline Atom & Atom & Atom & Angle/ ${ }^{\circ}$ & Atom & Atom & Atom & Angle/ ${ }^{\circ}$ \\
\hline $\mathrm{C}(3)$ & $\mathrm{C}(4)$ & $\mathrm{H}(4)$ & 120.8 & $\mathrm{O}(4)$ & $C(19)$ & $\mathrm{H}(19 \mathrm{~A})$ & 109.5 \\
\hline $\mathrm{C}(5)$ & $\mathrm{C}(4)$ & $\mathrm{H}(4)$ & 120.8 & $\mathrm{O}(4)$ & $C(19)$ & $\mathrm{H}(19 \mathrm{~B})$ & 109.5 \\
\hline$C(6)$ & $C(5)$ & $C(4)$ & $121.2(3)$ & $\mathrm{H}(19 \mathrm{~A})$ & $C(19)$ & $\mathrm{H}(19 B)$ & 109.5 \\
\hline$C(6)$ & $C(5)$ & $\mathrm{Cl}(2)$ & $120.5(2)$ & $\mathrm{O}(4)$ & $C(19)$ & $\mathrm{H}(19 \mathrm{C})$ & 109.5 \\
\hline $\mathrm{C}(4)$ & $\mathrm{C}(5)$ & $\mathrm{Cl}(2)$ & $118.3(2)$ & $\mathrm{H}(19 \mathrm{~A})$ & $C(19)$ & $\mathrm{H}(19 \mathrm{C})$ & 109.5 \\
\hline $\mathrm{C}(5)$ & $C(6)$ & $\mathrm{C}(1)$ & $117.3(3)$ & $\mathrm{H}(19 \mathrm{~B})$ & $\mathrm{C}(19)$ & $\mathrm{H}(19 \mathrm{C})$ & 109.5 \\
\hline $\mathrm{C}(5)$ & $C(6)$ & $C(7)$ & $133.3(3)$ & $\mathrm{C}(21)$ & $\mathrm{C}(20)$ & $C(7)$ & $113.3(3)$ \\
\hline $\mathrm{C}(1)$ & $\mathrm{C}(6)$ & $C(7)$ & $109.3(2)$ & $\mathrm{C}(21)$ & $\mathrm{C}(20)$ & $\mathrm{H}(20 \mathrm{~A})$ & 108.9 \\
\hline$C(6)$ & $C(7)$ & $\mathrm{C}(20)$ & $115.5(2)$ & $C(7)$ & $\mathrm{C}(20)$ & $H(20 A)$ & 108.9 \\
\hline $\mathrm{C}(6)$ & $\mathrm{C}(7)$ & $\mathrm{C}(8)$ & $101.3(2)$ & $\mathrm{C}(21)$ & $\mathrm{C}(20)$ & $\mathrm{H}(20 \mathrm{~B})$ & 108.9 \\
\hline $\mathrm{C}(20)$ & $\mathrm{C}(7)$ & $\mathrm{C}(8)$ & $109.5(2)$ & $\mathrm{C}(7)$ & $\mathrm{C}(20)$ & $\mathrm{H}(20 \mathrm{~B})$ & 108.9 \\
\hline$C(6)$ & $C(7)$ & $\mathrm{C}(9)$ & $110.4(2)$ & $\mathrm{H}(20 \mathrm{~A})$ & $\mathrm{C}(20)$ & $\mathrm{H}(20 \mathrm{~B})$ & 107.7 \\
\hline $\mathrm{C}(20)$ & $C(7)$ & $\mathrm{C}(9)$ & $109.0(3)$ & $C(22)$ & $\mathrm{C}(21)$ & $\mathrm{C}(20)$ & $126.3(7)$ \\
\hline $\mathrm{C}(8)$ & $\mathrm{C}(7)$ & $C(9)$ & $110.9(2)$ & $C\left(22^{\prime}\right)$ & $\mathrm{C}(21)$ & $\mathrm{C}(20)$ & $128.4(8)$ \\
\hline $\mathrm{O}(1)$ & $\mathrm{C}(8)$ & $\mathrm{N}(1)$ & $125.5(3)$ & $\mathrm{C}(22)$ & $\mathrm{C}(21)$ & $\mathrm{H}(21)$ & 116.9 \\
\hline $\mathrm{O}(1)$ & $C(8)$ & $C(7)$ & $126.5(3)$ & $C(20)$ & $\mathrm{C}(21)$ & $\mathrm{H}(21)$ & 116.8 \\
\hline $\mathrm{N}(1)$ & $\mathrm{C}(8)$ & $\mathrm{C}(7)$ & $108.0(3)$ & $\mathrm{C}\left(22^{\prime}\right)$ & $C(21)$ & $\mathrm{H}\left(21^{\prime}\right)$ & 115.8 \\
\hline $\mathrm{C}(11)$ & $\mathrm{C}(9)$ & $\mathrm{C}(10)$ & $108.8(3)$ & $\mathrm{C}(20)$ & $\mathrm{C}(21)$ & $\mathrm{H}\left(21^{\prime}\right)$ & 115.8 \\
\hline $\mathrm{C}(11)$ & $\mathrm{C}(9)$ & $C(7)$ & $112.3(3)$ & $\mathrm{C}(21)$ & $\mathrm{C}(22)$ & $\mathrm{H}(22 \mathrm{~A})$ & 120.0 \\
\hline$C(10)$ & $\mathrm{C}(9)$ & $C(7)$ & $111.9(3)$ & $\mathrm{C}(21)$ & $\mathrm{C}(22)$ & $\mathrm{H}(22 \mathrm{~B})$ & 120.0 \\
\hline $\mathrm{C}(11)$ & $\mathrm{C}(9)$ & $\mathrm{H}(9)$ & 107.9 & $\mathrm{H}(22 \mathrm{~A})$ & $\mathrm{C}(22)$ & $\mathrm{H}(22 \mathrm{~B})$ & 120.0 \\
\hline $\mathrm{C}(10)$ & $\mathrm{C}(9)$ & $\mathrm{H}(9)$ & 107.9 & $C(21)$ & $C\left(22^{\prime}\right)$ & $\mathrm{H}(22 \mathrm{C})$ & 120.0 \\
\hline $\mathrm{C}(7)$ & $\mathrm{C}(9)$ & $\mathrm{H}(9)$ & 107.9 & $C(21)$ & $C\left(22^{\prime}\right)$ & $H(22 D)$ & 120.0 \\
\hline $\mathrm{N}(2)$ & $\mathrm{C}(10)$ & $\mathrm{C}(9)$ & $110.2(3)$ & $\mathrm{H}(22 \mathrm{C})$ & $C\left(22^{\prime}\right)$ & $H(22 D)$ & 120.0 \\
\hline
\end{tabular}

Table S12. Hydrogen Atom Coordinates $\left(\AA \times 10^{4}\right)$ and Isotropic Displacement Parameters $\left(\AA^{2} \times 10^{3}\right)$ for $11 r$.

\begin{tabular}{ccccc}
\hline Atom & $\boldsymbol{x}$ & $\boldsymbol{y}$ & $\boldsymbol{z}$ & $\mathrm{U}(\mathbf{e q})$ \\
\hline $\mathrm{H}(2 \mathrm{~A})$ & 572 & -1389 & 7905 & 50
\end{tabular}




\begin{tabular}{ccccc}
$\mathrm{H}(4)$ & 1779 & 987 & 5720 & 54 \\
$\mathrm{H}(9)$ & 3293 & 4680 & 8922 & 49 \\
$\mathrm{H}(10 \mathrm{~A})$ & 3673 & 1924 & 10196 & 64 \\
$\mathrm{H}(10 \mathrm{~B})$ & 3867 & 2000 & 9054 & 64 \\
$\mathrm{H}(14)$ & 4894 & 7486 & 12679 & 69 \\
$\mathrm{H}(15)$ & 5153 & 7867 & 14492 & 69 \\
$\mathrm{H}(17)$ & 2166 & 4698 & 14225 & 68 \\
$\mathrm{H}(18)$ & 1960 & 4273 & 12443 & 63 \\
$\mathrm{H}(19 \mathrm{~A})$ & 5608 & 7344 & 16331 & 127 \\
$\mathrm{H}(19 \mathrm{~B})$ & 4575 & 7539 & 16979 & 127 \\
$\mathrm{H}(19 \mathrm{C})$ & 4398 & 8576 & 15951 & 127 \\
$\mathrm{H}(20 \mathrm{~A})$ & 583 & 5677 & 8922 & 56 \\
$\mathrm{H}(20 \mathrm{~B})$ & 979 & 5578 & 7865 & 56 \\
$\mathrm{H}(21)$ & -1450 & 4052 & 7989 & 90 \\
$\mathrm{H}(21)$ & -1090 & 3922 & 7079 & 90 \\
$\mathrm{H}(22 \mathrm{~A})$ & -2438 & 5607 & 6529 & 131 \\
$\mathrm{H}(22 \mathrm{~B})$ & -1177 & 4957 & 6165 & 131 \\
$\mathrm{H}(22 \mathrm{C})$ & -2708 & 5693 & 7145 & 111 \\
$\mathrm{H}(22 \mathrm{D})$ & -2145 & 5261 & 8424 & 111 \\
$\mathrm{H}(1)$ & $270(40)$ & $500(60)$ & $9460(30)$ & $61(12)$ \\
\hline
\end{tabular}

Table S13. Torsion Angles for 11r.

\begin{tabular}{ccccc}
\hline $\mathbf{A}$ & $\mathbf{B}$ & $\mathbf{C}$ & $\mathbf{D}$ & Angle/ $^{\mathbf{0}}$ \\
\hline $\mathrm{C}(8)$ & $\mathrm{N}(1)$ & $\mathrm{C}(1)$ & $\mathrm{C}(2)$ & $-177.2(3)$ \\
$\mathrm{C}(8)$ & $\mathrm{N}(1)$ & $\mathrm{C}(1)$ & $\mathrm{C}(6)$ & $2.3(4)$ \\
$\mathrm{C}(6)$ & $\mathrm{C}(1)$ & $\mathrm{C}(2)$ & $\mathrm{C}(3)$ & $-1.6(5)$ \\
$\mathrm{N}(1)$ & $\mathrm{C}(1)$ & $\mathrm{C}(2)$ & $\mathrm{C}(3)$ & $177.7(3)$ \\
$\mathrm{C}(1)$ & $\mathrm{C}(2)$ & $\mathrm{C}(3)$ & $\mathrm{C}(4)$ & $1.2(5)$ \\
$\mathrm{C}(1)$ & $\mathrm{C}(2)$ & $\mathrm{C}(3)$ & $\mathrm{Cl}(1)$ & $-177.9(2)$ \\
$\mathrm{C}(2)$ & $\mathrm{C}(3)$ & $\mathrm{C}(4)$ & $\mathrm{C}(5)$ & $0.5(5)$
\end{tabular}




\begin{tabular}{|c|c|c|c|c|}
\hline $\mathbf{A}$ & B & C & D & Angle/o \\
\hline $\mathrm{Cl}(1)$ & $\mathrm{C}(3)$ & $\mathrm{C}(4)$ & $C(5)$ & $179.6(2)$ \\
\hline $\mathrm{C}(3)$ & $\mathrm{C}(4)$ & $\mathrm{C}(5)$ & $C(6)$ & $-1.9(5)$ \\
\hline $\mathrm{C}(3)$ & $\mathrm{C}(4)$ & $\mathrm{C}(5)$ & $\mathrm{Cl}(2)$ & $177.9(2)$ \\
\hline $\mathrm{C}(4)$ & $\mathrm{C}(5)$ & $\mathrm{C}(6)$ & $C(1)$ & $1.6(4)$ \\
\hline $\mathrm{Cl}(2)$ & $\mathrm{C}(5)$ & $\mathrm{C}(6)$ & $\mathrm{C}(1)$ & $-178.3(2)$ \\
\hline $\mathrm{C}(4)$ & $\mathrm{C}(5)$ & $C(6)$ & $C(7)$ & $178.4(3)$ \\
\hline $\mathrm{Cl}(2)$ & $\mathrm{C}(5)$ & $\mathrm{C}(6)$ & $C(7)$ & $-1.4(5)$ \\
\hline $\mathrm{C}(2)$ & $\mathrm{C}(1)$ & $\mathrm{C}(6)$ & $C(5)$ & $0.3(5)$ \\
\hline $\mathrm{N}(1)$ & $\mathrm{C}(1)$ & $\mathrm{C}(6)$ & $C(5)$ & $-179.2(3)$ \\
\hline$C(2)$ & $\mathrm{C}(1)$ & $\mathrm{C}(6)$ & $C(7)$ & $-177.3(3)$ \\
\hline $\mathrm{N}(1)$ & $\mathrm{C}(1)$ & $\mathrm{C}(6)$ & $C(7)$ & $3.2(3)$ \\
\hline $\mathrm{C}(5)$ & $\mathrm{C}(6)$ & $\mathrm{C}(7)$ & $\mathrm{C}(20)$ & $58.1(5)$ \\
\hline $\mathrm{C}(1)$ & $\mathrm{C}(6)$ & $\mathrm{C}(7)$ & $\mathrm{C}(20)$ & $-124.9(3)$ \\
\hline$C(5)$ & $\mathrm{C}(6)$ & $\mathrm{C}(7)$ & $C(8)$ & $176.4(3)$ \\
\hline $\mathrm{C}(1)$ & $C(6)$ & $\mathrm{C}(7)$ & $\mathrm{C}(8)$ & $-6.6(3)$ \\
\hline $\mathrm{C}(5)$ & $\mathrm{C}(6)$ & $\mathrm{C}(7)$ & $\mathrm{C}(9)$ & $-66.1(4)$ \\
\hline $\mathrm{C}(1)$ & $\mathrm{C}(6)$ & $\mathrm{C}(7)$ & $C(9)$ & $110.9(3)$ \\
\hline $\mathrm{C}(1)$ & $\mathrm{N}(1)$ & $\mathrm{C}(8)$ & $\mathrm{O}(1)$ & $173.0(3)$ \\
\hline $\mathrm{C}(1)$ & $\mathrm{N}(1)$ & $\mathrm{C}(8)$ & $C(7)$ & $-6.6(4)$ \\
\hline$C(6)$ & $\mathrm{C}(7)$ & $\mathrm{C}(8)$ & $\mathrm{O}(1)$ & $-171.7(3)$ \\
\hline$C(20)$ & $\mathrm{C}(7)$ & $\mathrm{C}(8)$ & $\mathrm{O}(1)$ & $-49.2(4)$ \\
\hline $\mathrm{C}(9)$ & $C(7)$ & $\mathrm{C}(8)$ & $\mathrm{O}(1)$ & $71.1(4)$ \\
\hline$C(6)$ & $\mathrm{C}(7)$ & $\mathrm{C}(8)$ & $\mathrm{N}(1)$ & $7.9(3)$ \\
\hline$C(20)$ & $C(7)$ & $\mathrm{C}(8)$ & $\mathrm{N}(1)$ & $130.4(3)$ \\
\hline $\mathrm{C}(9)$ & $C(7)$ & $\mathrm{C}(8)$ & $\mathrm{N}(1)$ & $-109.3(3)$ \\
\hline$C(6)$ & $\mathrm{C}(7)$ & $\mathrm{C}(9)$ & $\mathrm{C}(11)$ & $-165.5(3)$ \\
\hline$C(20)$ & $C(7)$ & $\mathrm{C}(9)$ & $\mathrm{C}(11)$ & $66.5(3)$ \\
\hline $\mathrm{C}(8)$ & $C(7)$ & $\mathrm{C}(9)$ & $\mathrm{C}(11)$ & $-54.1(3)$ \\
\hline$C(6)$ & $\mathrm{C}(7)$ & $\mathrm{C}(9)$ & $\mathrm{C}(10)$ & $-42.8(3)$ \\
\hline
\end{tabular}




\begin{tabular}{ccccc}
\hline $\mathbf{A}$ & $\mathbf{B}$ & $\mathbf{C}$ & $\mathbf{D}$ & Angle/ $^{\mathbf{0}}$ \\
\hline $\mathrm{C}(20)$ & $\mathrm{C}(7)$ & $\mathrm{C}(9)$ & $\mathrm{C}(10)$ & $-170.7(3)$ \\
$\mathrm{C}(8)$ & $\mathrm{C}(7)$ & $\mathrm{C}(9)$ & $\mathrm{C}(10)$ & $68.6(3)$ \\
$\mathrm{O}(3)$ & $\mathrm{N}(2)$ & $\mathrm{C}(10)$ & $\mathrm{C}(9)$ & $-57.5(4)$ \\
$\mathrm{O}(2)$ & $\mathrm{N}(2)$ & $\mathrm{C}(10)$ & $\mathrm{C}(9)$ & $124.7(4)$ \\
$\mathrm{C}(11)$ & $\mathrm{C}(9)$ & $\mathrm{C}(10)$ & $\mathrm{N}(2)$ & $-59.2(4)$ \\
$\mathrm{C}(7)$ & $\mathrm{C}(9)$ & $\mathrm{C}(10)$ & $\mathrm{N}(2)$ & $176.1(3)$ \\
$\mathrm{C}(18)$ & $\mathrm{C}(13)$ & $\mathrm{C}(14)$ & $\mathrm{C}(15)$ & $2.0(6)$ \\
$\mathrm{C}(12)$ & $\mathrm{C}(13)$ & $\mathrm{C}(14)$ & $\mathrm{C}(15)$ & $-174.2(4)$ \\
$\mathrm{C}(13)$ & $\mathrm{C}(14)$ & $\mathrm{C}(15)$ & $\mathrm{C}(16)$ & $-1.6(6)$ \\
$\mathrm{C}(19)$ & $\mathrm{O}(4)$ & $\mathrm{C}(16)$ & $\mathrm{C}(15)$ & $5.4(6)$ \\
$\mathrm{C}(19)$ & $\mathrm{O}(4)$ & $\mathrm{C}(16)$ & $\mathrm{C}(17)$ & $-176.4(4)$ \\
$\mathrm{C}(14)$ & $\mathrm{C}(15)$ & $\mathrm{C}(16)$ & $\mathrm{O}(4)$ & $178.3(4)$ \\
$\mathrm{C}(14)$ & $\mathrm{C}(15)$ & $\mathrm{C}(16)$ & $\mathrm{C}(17)$ & $0.2(6)$ \\
$\mathrm{O}(4)$ & $\mathrm{C}(16)$ & $\mathrm{C}(17)$ & $\mathrm{C}(18)$ & $\mathrm{C}(18)$ \\
$\mathrm{C}(15)$ & $\mathrm{C}(16)$ & $\mathrm{C}(17)$ & $\mathrm{C}(18)$ & $0.8(6)$ \\
$\mathrm{C}(16)$ & $\mathrm{C}(17)$ & $\mathrm{C}(18)$ & $\mathrm{C}(13)$ & $-0.4(6)$ \\
$\mathrm{C}(14)$ & $\mathrm{C}(13)$ & $\mathrm{C}(18)$ & $\mathrm{C}(17)$ & $-1.0(6)$ \\
$\mathrm{C}(12)$ & $\mathrm{C}(13)$ & $\mathrm{C}(18)$ & $\mathrm{C}(17)$ & $175.4(4)$ \\
$\mathrm{C}(6)$ & $\mathrm{C}(7)$ & $\mathrm{C}(20)$ & $\mathrm{C}(21)$ & $53.0(4)$ \\
$\mathrm{C}(8)$ & $\mathrm{C}(7)$ & $\mathrm{C}(20)$ & $\mathrm{C}(21)$ & $-60.5(4)$ \\
$\mathrm{C}(9)$ & $\mathrm{C}(7)$ & $\mathrm{C}(20)$ & $\mathrm{C}(21)$ & $178.0(3)$ \\
$\mathrm{C}(7)$ & $\mathrm{C}(20)$ & $\mathrm{C}(21)$ & $\mathrm{C}(22)$ & $-124.8(9)$ \\
$\mathrm{C}(7)$ & $\mathrm{C}(20)$ & $\mathrm{C}(21)$ & $\mathrm{C}(22)$ & $129.0(11)$ \\
\hline & & & & \\
\hline
\end{tabular}


The solvent system of $n$-hexane/THF (4/1, v/v) was employed for crystal growth. The ellipsoid contour $50 \%$ probability levels in the caption for the image of the structure.

Data intensity of $\mathbf{1 3}^{2}$ was collected using a 'Bruker APEX-II CCD' diffractometer at 296(2) K. Data collection and reduction were done by using Olex2 and the structure was solved with the ShelXS structure solution program using direct methods and refined by full-matrix least-squares on $F^{2}$ with anisotropic displacement parameters for non-H atoms using SHELX-97. Hydrogen atoms were added at their geometrically idea positions and refined isotropically. Crystal data for 10: $\mathrm{C}_{25} \mathrm{H}_{20} \mathrm{~N}_{2} \mathrm{O}_{5} \mathrm{~S}, \mathrm{M}=$ 460.49, $T=193(2) \mathrm{K}, \lambda=0.71073 \AA$, Monoclinic, space group $\mathrm{P} 21 / \mathrm{c}, a=8.3647(10) \AA, b=$ 13.1827(15) $\AA, c=19.997(3) \AA, V=2205.0(5) \AA^{3}, \mathrm{Z}=4, d_{\text {calc }}=1.387 \mathrm{Mg} / \mathrm{m}^{3}, 11103$ reflections measured, $4308[\mathrm{R}($ int $)=0.0354], \mathrm{R}_{1}=0.0351, \mathrm{wR}_{2}=0.0803\left(I>2 \sigma(I)\right.$, final $\mathrm{R}_{1}=0.0415, \mathrm{wR}_{2}=$ $0.0851, \mathrm{GOF}=1.051$, and 299 parameters.
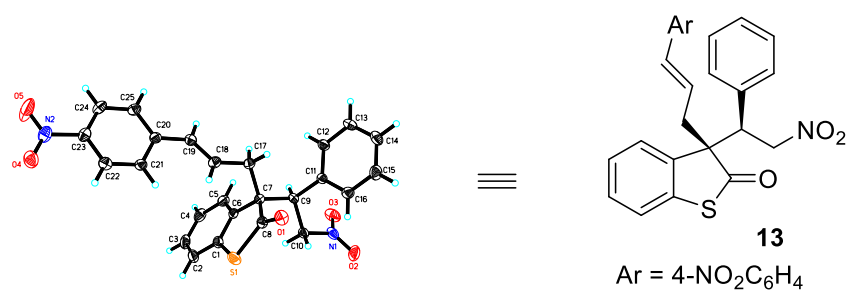

Table S14. Crystal data and structure refinement for 13.

Identification code

Empirical formula

Formula weight

Temperature

Wavelength

Crystal system

Space group

Unit cell dimensions
13

$\mathrm{C}_{25} \mathrm{H}_{20} \mathrm{~N}_{2} \mathrm{O}_{5} \mathrm{~S}$

460.49

193(2) K

$0.71073 \AA$

Orthorhombic

P 212121

$\mathrm{a}=8.3647(10) \AA \quad \mathrm{a}=90^{\circ}$.

$\mathrm{b}=13.1827(15) \AA \quad \mathrm{b}=90^{\circ}$.

$\mathrm{c}=19.997(3) \AA \quad \mathrm{g}=90^{\circ}$.
Volume

Z
2205.0(5) $\AA^{3}$

4

2 Supplementary crystallographic data have been deposited at the Cambridge Crystallographic Data Center. (CCDC2036953). 
Density (calculated)

Absorption coefficient

$\mathrm{F}(000)$

Crystal size

Theta range for data collection

Index ranges

Reflections collected

Independent reflections

Completeness to theta $=25.242^{\circ}$

Absorption correction

Max. and min. transmission

Refinement method

Data / restraints / parameters

Goodness-of-fit on $\mathrm{F}^{2}$

Final $\mathrm{R}$ indices [I $>2 \operatorname{sigma}(\mathrm{I})]$

$\mathrm{R}$ indices (all data)

Absolute structure parameter

Extinction coefficient

Largest diff. peak and hole
$1.387 \mathrm{Mg} / \mathrm{m}^{3}$

$0.188 \mathrm{~mm}^{-1}$

960

$0.190 \times 0.150 \times 0.100 \mathrm{~mm}^{3}$

2.557 to $25.996^{\circ}$.

$-10<=\mathrm{h}<=10,-16<=\mathrm{k}<=15,-24<=\mathrm{l}<=20$

11103

$4308[\mathrm{R}(\mathrm{int})=0.0354]$

$99.3 \%$

Semi-empirical from equivalents

0.7456 and 0.5885

Full-matrix least-squares on $\mathrm{F}^{2}$

4308 / 0 / 299

1.051

$\mathrm{R}_{1}=0.0351, \mathrm{wR}_{2}=0.0803$

$\mathrm{R}_{1}=0.0415, \mathrm{wR}_{2}=0.0851$

$0.06(4)$

0.011(3)

0.159 and -0.206 e. $\AA^{-3}$

Table S15. Atomic coordinates $\left(\times 10^{4}\right)$ and equivalent isotropic displacement parameters $\left(\AA^{2} \times 10^{3}\right)$ for 13. $\mathrm{U}(\mathrm{eq})$ is defined as one third of the trace of the orthogonalized Uij tensor.

\begin{tabular}{ccccc}
\hline Atom & $\boldsymbol{x}$ & $\boldsymbol{y}$ & $\boldsymbol{z}$ & $\mathbf{U}(\mathbf{e q})$ \\
\hline $\mathrm{S}(1)$ & $3890(1)$ & $3747(1)$ & $2956(1)$ & $45(1)$ \\
$\mathrm{O}(1)$ & $5798(2)$ & $3430(2)$ & $3980(1)$ & $44(1)$ \\
$\mathrm{O}(2)$ & $9618(3)$ & $1802(2)$ & $2300(1)$ & $64(1)$ \\
$\mathrm{O}(3)$ & $10656(2)$ & $3269(2)$ & $2102(1)$ & $61(1)$ \\
$\mathrm{O}(4)$ & $-1577(3)$ & $8799(2)$ & $4475(1)$ & $58(1)$ \\
$\mathrm{O}(5)$ & $-222(4)$ & $10158(2)$ & $4550(2)$ & $105(1)$ \\
$\mathrm{N}(1)$ & $9576(3)$ & $2730(2)$ & $2302(1)$ & $44(1)$
\end{tabular}




\begin{tabular}{|c|c|c|c|c|}
\hline Atom & $x$ & $y$ & $z$ & $\mathbf{U}(\mathbf{e q})$ \\
\hline $\mathrm{N}(2)$ & $-309(3)$ & $9252(2)$ & $4472(1)$ & $49(1)$ \\
\hline $\mathrm{C}(1)$ & $4523(3)$ & $4705(2)$ & $2405(1)$ & $36(1)$ \\
\hline $\mathrm{C}(2)$ & $3633(4)$ & $5068(2)$ & $1869(2)$ & $47(1)$ \\
\hline $\mathrm{C}(3)$ & $4281(4)$ & $5815(2)$ & $1472(2)$ & $52(1)$ \\
\hline $\mathrm{C}(4)$ & $5795(4)$ & $6196(2)$ & $1600(1)$ & $49(1)$ \\
\hline$C(5)$ & $6661(4)$ & $5843(2)$ & $2147(1)$ & $40(1)$ \\
\hline$C(6)$ & $6038(3)$ & $5094(2)$ & $2550(1)$ & $30(1)$ \\
\hline $\mathrm{C}(7)$ & $6801(3)$ & $4665(2)$ & $3177(1)$ & $28(1)$ \\
\hline $\mathrm{C}(8)$ & $5611(3)$ & $3891(2)$ & $3468(1)$ & $33(1)$ \\
\hline $\mathrm{C}(9)$ & $8422(3)$ & $4122(2)$ & $3003(1)$ & $28(1)$ \\
\hline$C(10)$ & $8070(3)$ & $3233(2)$ & $2535(1)$ & $35(1)$ \\
\hline $\mathrm{C}(11)$ & $9437(3)$ & $3816(2)$ & $3605(1)$ & $28(1)$ \\
\hline$C(12)$ & $10490(3)$ & $4519(2)$ & $3870(1)$ & $37(1)$ \\
\hline$C(13)$ & $11457(3)$ & $4282(2)$ & $4414(2)$ & $45(1)$ \\
\hline$C(14)$ & $11410(3)$ & $3325(2)$ & $4683(1)$ & $46(1)$ \\
\hline $\mathrm{C}(15)$ & $10389(4)$ & $2619(2)$ & $4426(2)$ & $49(1)$ \\
\hline$C(16)$ & $9398(3)$ & $2858(2)$ & $3888(1)$ & $39(1)$ \\
\hline$C(17)$ & $7049(3)$ & $5500(2)$ & $3709(1)$ & $35(1)$ \\
\hline $\mathrm{C}(18)$ & $5546(3)$ & $6064(2)$ & 3871(1) & $37(1)$ \\
\hline$C(19)$ & $5403(3)$ & $7050(2)$ & $3937(1)$ & $38(1)$ \\
\hline$C(20)$ & $3937(3)$ & $7602(2)$ & $4110(1)$ & $33(1)$ \\
\hline $\mathrm{C}(21)$ & $2440(3)$ & $7126(2)$ & 4111(1) & $38(1)$ \\
\hline$C(22)$ & $1068(4)$ & $7655(2)$ & $4249(1)$ & $38(1)$ \\
\hline$C(23)$ & $1168(4)$ & $8678(2)$ & $4372(1)$ & $37(1)$ \\
\hline $\mathrm{C}(24)$ & $2630(4)$ & $9174(2)$ & 4389(1) & $44(1)$ \\
\hline$C(25)$ & $3992(3)$ & $8633(2)$ & $4262(1)$ & $38(1)$ \\
\hline
\end{tabular}

Table S16. Bond Lengths for 13.

\begin{tabular}{llllll} 
Atom Atom Length/Å Atom Atom Length/A \\
\hline
\end{tabular}




\begin{tabular}{|c|c|c|c|c|c|}
\hline $\mathrm{S}(1)$ & $\mathrm{C}(1)$ & $1.758(3)$ & $\mathrm{C}(11)$ & $C(16)$ & $1.384(4)$ \\
\hline $\mathrm{S}(1)$ & $\mathrm{C}(8)$ & $1.776(3)$ & $C(12)$ & $C(13)$ & $1.391(4)$ \\
\hline $\mathrm{O}(1)$ & $\mathrm{C}(8)$ & $\mathrm{C}(8)$ & $C(12)$ & $\mathrm{H}(12)$ & 0.9500 \\
\hline $\mathrm{O}(2)$ & $\mathrm{N}(1)$ & $1.223(3)$ & $C(13)$ & $\mathrm{C}(14)$ & $C(14)$ \\
\hline $\mathrm{O}(3)$ & $\mathrm{N}(1)$ & $1.217(3)$ & $C(13)$ & $\mathrm{H}(13)$ & 0.9500 \\
\hline $\mathrm{O}(4)$ & $\mathrm{N}(2)$ & $\mathrm{N}(2)$ & $C(14)$ & $C(15)$ & $1.364(4)$ \\
\hline $\mathrm{O}(5)$ & $\mathrm{N}(2)$ & $1.207(3)$ & $C(14)$ & $\mathrm{H}(14)$ & 0.9500 \\
\hline $\mathrm{N}(1)$ & $C(10)$ & $1.498(3)$ & $C(15)$ & $C(16)$ & $1.393(4)$ \\
\hline $\mathrm{N}(2)$ & $C(23)$ & $1.463(4)$ & $C(15)$ & $\mathrm{H}(15)$ & 0.9500 \\
\hline $\mathrm{C}(1)$ & $\mathrm{C}(2)$ & $1.390(4)$ & $C(17)$ & $\mathrm{C}(18)$ & $1.495(4)$ \\
\hline $\mathrm{C}(1)$ & $\mathrm{C}(6)$ & $1.398(4)$ & $C(17)$ & $\mathrm{H}(17 \mathrm{~A})$ & 0.9900 \\
\hline $\mathrm{C}(2)$ & $\mathrm{C}(3)$ & $1.375(4)$ & $C(17)$ & $\mathrm{H}(17 \mathrm{~B})$ & 0.9900 \\
\hline $\mathrm{C}(2)$ & $\mathrm{H}(2)$ & 0.9500 & $\mathrm{C}(18)$ & C(19) & $1.312(4)$ \\
\hline $\mathrm{C}(3)$ & $\mathrm{C}(4)$ & $1.386(5)$ & $\mathrm{C}(18)$ & $\mathrm{H}(18)$ & 0.9500 \\
\hline $\mathrm{C}(3)$ & $\mathrm{H}(3)$ & 0.9500 & $\mathrm{C}(19)$ & $C(20)$ & $1.467(4)$ \\
\hline $\mathrm{C}(4)$ & $\mathrm{C}(5)$ & $1.393(4)$ & $C(19)$ & H(19) & 0.9500 \\
\hline $\mathrm{C}(4)$ & $\mathrm{H}(4)$ & 0.9500 & $C(20)$ & $C(25)$ & $1.394(4)$ \\
\hline$C(5)$ & $C(6)$ & $1.378(4)$ & $\mathrm{C}(20)$ & $C(21)$ & $1.400(4)$ \\
\hline$C(5)$ & $\mathrm{H}(5)$ & 0.9500 & $C(21)$ & $C(22)$ & $1.372(4)$ \\
\hline$C(6)$ & $\mathrm{C}(7)$ & $1.516(3)$ & $\mathrm{C}(21)$ & $\mathrm{H}(21)$ & 0.9500 \\
\hline$C(7)$ & $\mathrm{C}(8)$ & $1.539(4)$ & $\mathrm{C}(22)$ & $C(23)$ & $1.374(4)$ \\
\hline$C(7)$ & $C(17)$ & $1.545(3)$ & $C(22)$ & $\mathrm{H}(22)$ & 0.9500 \\
\hline$C(7)$ & $\mathrm{C}(9)$ & $1.572(3)$ & $C(23)$ & $C(24)$ & $1.387(4)$ \\
\hline $\mathrm{C}(9)$ & $\mathrm{C}(11)$ & $1.527(3)$ & $C(24)$ & $C(25)$ & $1.368(4)$ \\
\hline $\mathrm{C}(9)$ & $C(10)$ & $1.529(3)$ & $C(24)$ & $\mathrm{H}(24)$ & 0.9500 \\
\hline $\mathrm{C}(9)$ & $\mathrm{H}(9)$ & 1.0000 & $C(25)$ & $\mathrm{H}(25)$ & 0.9500 \\
\hline$C(10)$ & $\mathrm{H}(10 \mathrm{~A})$ & 0.9900 & & & \\
\hline$C(10)$ & $\mathrm{H}(10 \mathrm{~B})$ & 0.9900 & & & \\
\hline$C(11)$ & $C(12)$ & $1.384(4)$ & & & \\
\hline
\end{tabular}

Table S17. Bond Angles for 13. 


\begin{tabular}{|c|c|c|c|c|c|c|c|}
\hline Atom & Atom & Atom & Angle/ ${ }^{\circ}$ & Atom & Atom & Atom & Angle/ ${ }^{\circ}$ \\
\hline $\mathrm{C}(1)$ & $\mathrm{S}(1)$ & $\mathrm{C}(8)$ & $92.30(12)$ & $\mathrm{C}(12)$ & $\mathrm{C}(11)$ & $\mathrm{C}(9)$ & $118.5(2)$ \\
\hline $\mathrm{O}(3)$ & $\mathrm{N}(1)$ & $\mathrm{O}(2)$ & $124.2(3)$ & $C(16)$ & $\mathrm{C}(11)$ & $\mathrm{C}(9)$ & $123.4(2)$ \\
\hline $\mathrm{O}(3)$ & $\mathrm{N}(1)$ & $\mathrm{C}(10)$ & $117.8(3)$ & $\mathrm{C}(11)$ & $\mathrm{C}(12)$ & $\mathrm{C}(13)$ & $121.2(3)$ \\
\hline $\mathrm{O}(2)$ & $\mathrm{N}(1)$ & $\mathrm{C}(10)$ & $117.9(3)$ & $\mathrm{C}(11)$ & $\mathrm{C}(12)$ & $\mathrm{H}(12)$ & 119.4 \\
\hline $\mathrm{O}(5)$ & $\mathrm{N}(2)$ & $\mathrm{O}(4)$ & $122.5(3)$ & $\mathrm{C}(13)$ & $\mathrm{C}(12)$ & $\mathrm{H}(12)$ & 119.4 \\
\hline $\mathrm{O}(5)$ & $\mathrm{N}(2)$ & $\mathrm{C}(23)$ & $118.6(3)$ & $C(14)$ & $\mathrm{C}(13)$ & $\mathrm{C}(12)$ & $119.8(3)$ \\
\hline $\mathrm{O}(4)$ & $\mathrm{N}(2)$ & $\mathrm{C}(23)$ & $118.9(2)$ & $C(14)$ & $\mathrm{C}(13)$ & $\mathrm{H}(13)$ & 120.1 \\
\hline $\mathrm{C}(2)$ & $\mathrm{C}(1)$ & $C(6)$ & $121.3(3)$ & $\mathrm{C}(12)$ & $\mathrm{C}(13)$ & $\mathrm{H}(13)$ & 120.1 \\
\hline $\mathrm{C}(2)$ & $\mathrm{C}(1)$ & $\mathrm{S}(1)$ & $124.7(2)$ & $\mathrm{C}(15)$ & $\mathrm{C}(14)$ & $\mathrm{C}(13)$ & $119.8(3)$ \\
\hline $\mathrm{C}(6)$ & $\mathrm{C}(1)$ & $\mathrm{S}(1)$ & $113.96(19)$ & $\mathrm{C}(15)$ & $\mathrm{C}(14)$ & $\mathrm{H}(14)$ & 120.1 \\
\hline $\mathrm{C}(3)$ & $\mathrm{C}(2)$ & $\mathrm{C}(1)$ & $118.7(3)$ & $\mathrm{H}(10 \mathrm{~A})$ & $\mathrm{C}(10)$ & $\mathrm{H}(10 \mathrm{~B})$ & 108.0 \\
\hline $\mathrm{C}(3)$ & $\mathrm{C}(2)$ & $\mathrm{H}(2)$ & 120.7 & $\mathrm{C}(12)$ & $\mathrm{C}(11)$ & $\mathrm{C}(16)$ & $118.0(2)$ \\
\hline $\mathrm{C}(1)$ & $\mathrm{C}(2)$ & $\mathrm{H}(2)$ & 120.7 & $C(12)$ & $\mathrm{C}(11)$ & $\mathrm{C}(9)$ & $118.5(2)$ \\
\hline $\mathrm{C}(2)$ & $\mathrm{C}(3)$ & $\mathrm{C}(4)$ & $120.9(3)$ & $C(16)$ & $\mathrm{C}(11)$ & $\mathrm{C}(9)$ & $123.4(2)$ \\
\hline $\mathrm{C}(2)$ & $\mathrm{C}(3)$ & $\mathrm{H}(3)$ & 119.5 & $\mathrm{C}(11)$ & $\mathrm{C}(12)$ & $\mathrm{C}(13)$ & $121.2(3)$ \\
\hline $\mathrm{C}(4)$ & $\mathrm{C}(3)$ & $\mathrm{H}(3)$ & 119.5 & $\mathrm{C}(11)$ & $\mathrm{C}(12)$ & $\mathrm{H}(12)$ & 119.4 \\
\hline $\mathrm{C}(3)$ & $\mathrm{C}(4)$ & $\mathrm{C}(5)$ & $119.9(3)$ & $\mathrm{C}(13)$ & $\mathrm{C}(12)$ & $\mathrm{H}(12)$ & 119.4 \\
\hline $\mathrm{C}(3)$ & $\mathrm{C}(4)$ & $\mathrm{H}(4)$ & 120.1 & $C(14)$ & $C(13)$ & $\mathrm{C}(12)$ & $119.8(3)$ \\
\hline$C(5)$ & $\mathrm{C}(4)$ & $\mathrm{H}(4)$ & 120.1 & $C(14)$ & $C(13)$ & $\mathrm{H}(13)$ & 120.1 \\
\hline$C(6)$ & $\mathrm{C}(5)$ & $\mathrm{C}(4)$ & $120.2(3)$ & $\mathrm{C}(12)$ & $\mathrm{C}(13)$ & $\mathrm{H}(13)$ & 120.1 \\
\hline$C(6)$ & $\mathrm{C}(5)$ & $\mathrm{H}(5)$ & 119.9 & $C(15)$ & $\mathrm{C}(14)$ & $\mathrm{C}(13)$ & $119.8(3)$ \\
\hline $\mathrm{C}(4)$ & $\mathrm{C}(5)$ & $\mathrm{H}(5)$ & 119.9 & $C(15)$ & $\mathrm{C}(14)$ & $\mathrm{H}(14)$ & 120.1 \\
\hline $\mathrm{C}(5)$ & $C(6)$ & $\mathrm{C}(1)$ & $118.9(2)$ & $\mathrm{C}(13)$ & $\mathrm{C}(14)$ & $\mathrm{H}(14)$ & 120.1 \\
\hline $\mathrm{C}(5)$ & $\mathrm{C}(6)$ & $\mathrm{C}(7)$ & $126.4(2)$ & $\mathrm{C}(14)$ & $\mathrm{C}(15)$ & $C(16)$ & $120.7(3)$ \\
\hline $\mathrm{C}(1)$ & $\mathrm{C}(6)$ & $\mathrm{C}(7)$ & $114.6(2)$ & $C(14)$ & $\mathrm{C}(15)$ & $\mathrm{H}(15)$ & 119.7 \\
\hline$C(6)$ & $\mathrm{C}(7)$ & $\mathrm{C}(8)$ & $106.7(2)$ & $C(16)$ & $\mathrm{C}(15)$ & $\mathrm{H}(15)$ & 119.7 \\
\hline $\mathrm{C}(6)$ & $\mathrm{C}(7)$ & $\mathrm{C}(17)$ & $111.08(19)$ & $\mathrm{C}(11)$ & $C(16)$ & $\mathrm{C}(15)$ & $120.5(3)$ \\
\hline $\mathrm{C}(8)$ & $\mathrm{C}(7)$ & $\mathrm{C}(17)$ & $107.4(2)$ & $\mathrm{C}(11)$ & $C(16)$ & $\mathrm{H}(16)$ & 119.8 \\
\hline$C(6)$ & $\mathrm{C}(7)$ & $\mathrm{C}(9)$ & $110.5(2)$ & $C(15)$ & $C(16)$ & $\mathrm{H}(16)$ & 119.8 \\
\hline
\end{tabular}




\begin{tabular}{|c|c|c|c|c|c|c|c|}
\hline Atom & Atom & Atom & Angle/ ${ }^{\circ}$ & Atom & Atom & Atom & Angle/o \\
\hline $\mathrm{C}(8)$ & $C(7)$ & $\mathrm{C}(9)$ & $109.88(19)$ & $\mathrm{C}(18)$ & $\mathrm{C}(17)$ & $C(7)$ & $113.0(2)$ \\
\hline$C(17)$ & $\mathrm{C}(7)$ & $C(9)$ & $111.14(19)$ & $\mathrm{C}(18)$ & $\mathrm{C}(17)$ & $\mathrm{H}(17 \mathrm{~A})$ & 109.0 \\
\hline $\mathrm{O}(1)$ & $\mathrm{C}(8)$ & $\mathrm{C}(7)$ & $124.9(2)$ & $\mathrm{C}(7)$ & $\mathrm{C}(17)$ & $\mathrm{H}(17 \mathrm{~A})$ & 109.0 \\
\hline $\mathrm{O}(1)$ & $\mathrm{C}(8)$ & $\mathrm{S}(1)$ & $122.9(2)$ & $\mathrm{C}(18)$ & $\mathrm{C}(17)$ & $\mathrm{H}(17 \mathrm{~B})$ & 109.0 \\
\hline $\mathrm{C}(7)$ & $\mathrm{C}(8)$ & $S(7)$ & $112.20(18)$ & $\mathrm{C}(7)$ & $\mathrm{C}(17)$ & $\mathrm{H}(17 \mathrm{~B})$ & 109.0 \\
\hline $\mathrm{C}(11)$ & $\mathrm{C}(9)$ & $\mathrm{C}(10)$ & $112.8(2)$ & $\mathrm{H}(17 \mathrm{~A})$ & $\mathrm{C}(17)$ & $\mathrm{H}(17 \mathrm{~B})$ & 107.8 \\
\hline $\mathrm{C}(11)$ & $\mathrm{C}(9)$ & $\mathrm{C}(7)$ & $115.2(2)$ & $C(19)$ & $\mathrm{C}(18)$ & $C(17)$ & $126.2(3)$ \\
\hline $\mathrm{C}(10)$ & $\mathrm{C}(9)$ & $\mathrm{C}(7)$ & $108.53(18)$ & $C(19)$ & $\mathrm{C}(18)$ & $\mathrm{H}(18)$ & 116.9 \\
\hline $\mathrm{C}(11)$ & $\mathrm{C}(9)$ & $\mathrm{C}(9)$ & 106.6 & $C(17)$ & $\mathrm{C}(18)$ & $\mathrm{H}(18)$ & 116.9 \\
\hline $\mathrm{C}(10)$ & $\mathrm{C}(9)$ & $\mathrm{C}(9)$ & 106.6 & $\mathrm{C}(18)$ & $C(19)$ & $C(20)$ & $126.2(3)$ \\
\hline $\mathrm{C}(7)$ & $\mathrm{C}(9)$ & $\mathrm{H}(9)$ & 106.6 & $\mathrm{C}(18)$ & $\mathrm{C}(19)$ & $\mathrm{H}(19)$ & 116.9 \\
\hline $\mathrm{N}(1)$ & $C(10)$ & $\mathrm{C}(9)$ & $111.6(2)$ & $C(20)$ & $C(19)$ & $\mathrm{H}(19)$ & 116.9 \\
\hline $\mathrm{C}(18)$ & 109.0 & $\mathrm{H}(17 \mathrm{~B})$ & 109.0 & $C(25)$ & $\mathrm{C}(20)$ & $\mathrm{C}(21)$ & $117.8(3)$ \\
\hline $\mathrm{C}(7)$ & 109.0 & $\mathrm{H}(17 \mathrm{~B})$ & 109.0 & $C(25)$ & $\mathrm{C}(20)$ & $C(19)$ & $120.5(3)$ \\
\hline $\mathrm{H}(17 \mathrm{~A})$ & 107.8 & $\mathrm{H}(17 \mathrm{~B})$ & 107.8 & $\mathrm{C}(21)$ & $C(20)$ & $C(19)$ & $121.7(2)$ \\
\hline$C(19)$ & $126.2(3)$ & $C(17)$ & $126.2(3)$ & $\mathrm{C}(22)$ & $\mathrm{C}(21)$ & $\mathrm{C}(20)$ & $121.4(2)$ \\
\hline $\mathrm{C}(19)$ & 116.9 & $\mathrm{H}(18)$ & 116.9 & $C(22)$ & $\mathrm{C}(21)$ & $\mathrm{H}(21)$ & 119.3 \\
\hline$C(17)$ & 116.9 & $\mathrm{H}(18)$ & 116.9 & $C(20)-$ & $\mathrm{C}(21)$ & $\mathrm{H}(21)$ & 119.3 \\
\hline $\mathrm{C}(18)$ & $126.2(3)$ & $C(20)$ & $126.2(3)$ & $\mathrm{C}(21)$ & $C(22)$ & $\mathrm{C}(23)$ & $119.0(3)$ \\
\hline $\mathrm{C}(18)$ & 116.9 & $\mathrm{H}(19)$ & 116.9 & $\mathrm{C}(21)$ & $C(22)$ & $\mathrm{H}(22)$ & 120.5 \\
\hline$C(20)$ & 116.9 & $\mathrm{H}(19)$ & 116.9 & $\mathrm{C}(23)$ & $C(22)$ & $\mathrm{H}(22)$ & 120.5 \\
\hline$C(25)$ & $117.8(3)$ & $C(21)$ & $117.8(3)$ & $\mathrm{C}(22)$ & $C(23)$ & $C(24)$ & $121.4(3)$ \\
\hline $\mathrm{C}(25)$ & $120.5(3)$ & $C(19)$ & $120.5(3)$ & $C(22)$ & $C(23)$ & $\mathrm{N}(2)$ & $118.7(3)$ \\
\hline $\mathrm{C}(21)$ & $121.7(2)$ & $C(19)$ & $121.7(2)$ & $C(24)$ & $C(23)$ & $\mathrm{N}(2)$ & $119.9(2)$ \\
\hline$C(22)$ & $121.4(2)$ & $C(20)$ & $121.4(2)$ & $C(25)$ & $C(24)$ & $\mathrm{C}(23)$ & $119.0(2)$ \\
\hline $\mathrm{N}(1)$ & $C(10)$ & $\mathrm{H}(10 \mathrm{~A})$ & 109.3 & $C(25)$ & $\mathrm{C}(24)$ & $\mathrm{H}(24)$ & 120.5 \\
\hline$C(9)$ & $C(10)$ & $\mathrm{H}(10 \mathrm{~A})$ & 109.3 & $\mathrm{C}(23)$ & $C(24)$ & $\mathrm{H}(24)$ & 120.5 \\
\hline $\mathrm{N}(1)$ & $C(10)$ & $\mathrm{H}(10 \mathrm{~B})$ & 109.3 & $C(24)$ & $C(25)$ & $C(20)$ & $121.4(3)$ \\
\hline$C(9)$ & $C(10)$ & $\mathrm{H}(10 \mathrm{~B})$ & 109.3 & $C(24)$ & $C(25)$ & $\mathrm{H}(25)$ & 119.3 \\
\hline
\end{tabular}




\begin{tabular}{cccc|cccc}
\hline Atom & Atom & Atom & Angle/ $^{\circ}$ & Atom & Atom & Atom & Angle/ $^{\mathbf{0}}$ \\
\hline $\mathrm{H}(10 \mathrm{~A})$ & $\mathrm{C}(10)$ & $\mathrm{H}(10 \mathrm{~B})$ & 108.0 & $\mathrm{C}(20)$ & $\mathrm{C}(25)$ & $\mathrm{H}(25)$ & 119.3 \\
$\mathrm{C}(12)$ & $\mathrm{C}(11)$ & $\mathrm{C}(16)$ & $118.0(2)$ & & & & \\
\hline
\end{tabular}

Table S18. Anisotropic Displacement Parameters $\left(\AA^{2} \times 10^{3}\right)$ for 13. The Anisotropic displacement factor exponent takes the form: $-2 \pi^{2}\left[h^{2} a^{* 2} U_{11}+2 h k a * b * U_{12}+\ldots\right]$.

\begin{tabular}{|c|c|c|c|c|c|c|}
\hline Atom & $\mathrm{U}_{11}$ & $\mathbf{U}_{22}$ & $\mathbf{U}_{33}$ & $\mathbf{U}_{23}$ & $\mathbf{U}_{13}$ & $\mathbf{U}_{12}$ \\
\hline $\mathrm{S}(1)$ & $30(1)$ & $52(1)$ & $54(1)$ & $6(1)$ & $-4(1)$ & $-8(1)$ \\
\hline $\mathrm{O}(1)$ & $44(1)$ & $49(1)$ & $40(1)$ & 11(1) & $4(1)$ & $-2(1)$ \\
\hline $\mathrm{O}(2)$ & $70(2)$ & $53(1)$ & $68(2)$ & $-14(1)$ & $-6(1)$ & $30(1)$ \\
\hline $\mathrm{O}(3)$ & $36(1)$ & $94(2)$ & $54(1)$ & $-22(1)$ & $5(1)$ & $-1(1)$ \\
\hline $\mathrm{O}(4)$ & $44(1)$ & $67(2)$ & $61(1)$ & $-2(1)$ & $8(1)$ & $6(1)$ \\
\hline $\mathrm{O}(5)$ & $76(2)$ & $43(2)$ & 195(4) & $-17(2)$ & $48(2)$ & $10(1)$ \\
\hline $\mathrm{N}(1)$ & $35(1)$ & $64(2)$ & $32(1)$ & $-13(1)$ & $-7(1)$ & 11(1) \\
\hline $\mathrm{N}(2)$ & $52(2)$ & $45(2)$ & $51(2)$ & $1(1)$ & $15(1)$ & $6(1)$ \\
\hline $\mathrm{C}(1)$ & $33(1)$ & $38(1)$ & $37(1)$ & $-3(1)$ & $-2(1)$ & $8(1)$ \\
\hline $\mathrm{C}(2)$ & $41(2)$ & $52(2)$ & $48(2)$ & $-6(1)$ & $-13(1)$ & $14(1)$ \\
\hline $\mathrm{C}(3)$ & $65(2)$ & $48(2)$ & $42(2)$ & $-1(1)$ & $-13(2)$ & $26(2)$ \\
\hline $\mathrm{C}(4)$ & $75(2)$ & $36(2)$ & $35(1)$ & $5(1)$ & $0(2)$ & $11(2)$ \\
\hline $\mathrm{C}(5)$ & $48(2)$ & $32(1)$ & $38(2)$ & $-1(1)$ & $-2(1)$ & $0(1)$ \\
\hline$C(6)$ & $33(1)$ & $27(1)$ & $30(1)$ & $-4(1)$ & $-2(1)$ & $6(1)$ \\
\hline $\mathrm{C}(7)$ & $28(1)$ & $27(1)$ & $30(1)$ & $-1(1)$ & $-1(1)$ & $1(1)$ \\
\hline $\mathrm{C}(8)$ & $30(1)$ & $34(1)$ & $35(1)$ & $-2(1)$ & 1(1) & $4(1)$ \\
\hline $\mathrm{C}(9)$ & $24(1)$ & $32(1)$ & $29(1)$ & $0(1)$ & $-2(1)$ & $-1(1)$ \\
\hline$C(10)$ & $28(1)$ & $42(2)$ & $35(1)$ & $-10(1)$ & $-4(1)$ & $7(1)$ \\
\hline $\mathrm{C}(11)$ & $26(1)$ & $32(1)$ & $27(1)$ & $-1(1)$ & $1(1)$ & $1(1)$ \\
\hline $\mathrm{C}(12)$ & $34(1)$ & $37(1)$ & $40(1)$ & $3(1)$ & $-6(1)$ & $-5(1)$ \\
\hline$C(13)$ & $38(2)$ & $54(2)$ & $44(2)$ & $-5(1)$ & $-13(1)$ & $-6(1)$ \\
\hline$C(14)$ & $42(2)$ & $61(2)$ & $34(2)$ & $3(1)$ & $-8(1)$ & $8(2)$ \\
\hline$C(15)$ & $55(2)$ & $43(2)$ & $47(2)$ & $12(1)$ & $-4(2)$ & $4(2)$ \\
\hline
\end{tabular}




\begin{tabular}{lllllll}
$\mathrm{C}(16)$ & $42(2)$ & $34(1)$ & $42(2)$ & $2(1)$ & $-5(1)$ & $-4(1)$ \\
$\mathrm{C}(17)$ & $38(1)$ & $34(1)$ & $32(1)$ & $-6(1)$ & $-3(1)$ & $3(1)$ \\
$\mathrm{C}(18)$ & $40(1)$ & $35(1)$ & $35(1)$ & $-5(1)$ & $4(1)$ & $-1(1)$ \\
$\mathrm{C}(19)$ & $36(1)$ & $35(2)$ & $43(2)$ & $-1(1)$ & $-7(1)$ & $-2(1)$ \\
$\mathrm{C}(20)$ & $41(1)$ & $30(1)$ & $29(1)$ & $1(1)$ & $-6(1)$ & $0(1)$ \\
$\mathrm{C}(21)$ & $40(2)$ & $28(1)$ & $46(2)$ & $0(1)$ & $-11(1)$ & $-2(1)$ \\
$\mathrm{C}(22)$ & $40(1)$ & $37(1)$ & $37(1)$ & $7(1)$ & $-2(1)$ & $-5(1)$ \\
$\mathrm{C}(23)$ & $45(1)$ & $36(1)$ & $31(1)$ & $2(1)$ & $8(1)$ & $4(1)$ \\
$\mathrm{C}(24)$ & $56(2)$ & $28(1)$ & $47(2)$ & $-4(1)$ & $11(2)$ & $-1(1)$ \\
$\mathrm{C}(25)$ & $42(2)$ & $31(1)$ & $42(1)$ & $-2(1)$ & $3(1)$ & $-7(1)$ \\
\hline
\end{tabular}

Table S19. Atomic coordinates $\left(\times 10^{4}\right)$ and equivalent isotropic displacement parameters $\left(\AA^{2} \times 10^{3}\right)$ for 13. $U(e q)$ is defined as one third of the trace of the orthogonalized Uij tensor.

\begin{tabular}{|c|c|c|c|c|}
\hline Atom & $x$ & $y$ & $z$ & $\mathrm{U}(\mathbf{e q})$ \\
\hline $\mathrm{H}(2)$ & 2598 & 4805 & 1778 & 57 \\
\hline $\mathrm{H}(3)$ & 3683 & 6072 & 1106 & 62 \\
\hline $\mathrm{H}(4)$ & 6240 & 6698 & 1314 & 59 \\
\hline $\mathrm{H}(5)$ & 7685 & 6120 & 2242 & 48 \\
\hline $\mathrm{H}(9)$ & 9071 & 4620 & 2741 & 34 \\
\hline $\mathrm{H}(10 \mathrm{~A})$ & 7396 & 2730 & 2771 & 42 \\
\hline $\mathrm{H}(10 \mathrm{~B})$ & 7465 & 3482 & 2142 & 42 \\
\hline $\mathrm{H}(12)$ & 10553 & 5176 & 3677 & 44 \\
\hline $\mathrm{H}(13)$ & 12148 & 4781 & 4598 & 54 \\
\hline $\mathrm{H}(14)$ & 12086 & 3155 & 5048 & 55 \\
\hline $\mathrm{H}(15)$ & 10353 & 1958 & 4614 & 58 \\
\hline $\mathrm{H}(16)$ & 8691 & 2359 & 3714 & 47 \\
\hline $\mathrm{H}(17 \mathrm{~A})$ & 7470 & 5186 & 4123 & 42 \\
\hline $\mathrm{H}(17 \mathrm{~B})$ & 7859 & 5987 & 3545 & 42 \\
\hline $\mathrm{H}(18)$ & 4606 & 5670 & 3933 & 44 \\
\hline $\mathrm{H}(19)$ & 6338 & 7444 & 3865 & 46 \\
\hline
\end{tabular}




\begin{tabular}{ccccc}
\hline Atom & $\boldsymbol{x}$ & $\boldsymbol{y}$ & $\boldsymbol{z}$ & $\mathbf{U}(\mathbf{e q})$ \\
\hline $\mathrm{H}(21)$ & 2375 & 6422 & 4014 & 46 \\
$\mathrm{H}(22)$ & 63 & 7320 & 4258 & 46 \\
$\mathrm{H}(24)$ & 2685 & 9878 & 4488 & 52 \\
$\mathrm{H}(25)$ & 4997 & 8968 & 4277 & 46 \\
\hline
\end{tabular}

Table S20. Torsion Angles for 13.

\begin{tabular}{ccccc}
\hline $\mathbf{A}$ & $\mathbf{B}$ & $\mathbf{C}$ & $\mathbf{D}$ & Angle/ \\
\hline $\mathrm{C}(8)$ & $\mathrm{S}(1)$ & $\mathrm{C}(1)$ & $\mathrm{C}(2)$ & $-175.3(3)$ \\
$\mathrm{C}(8)$ & $\mathrm{S}(1)$ & $\mathrm{C}(1)$ & $\mathrm{C}(6)$ & $4.5(2)$ \\
$\mathrm{C}(6)$ & $\mathrm{C}(1)$ & $\mathrm{C}(2)$ & $\mathrm{C}(3)$ & $0.9(4)$ \\
$\mathrm{S}(1)$ & $\mathrm{C}(1)$ & $\mathrm{C}(2)$ & $\mathrm{C}(3)$ & $-179.3(2)$ \\
$\mathrm{C}(1)$ & $\mathrm{C}(2)$ & $\mathrm{C}(3)$ & $\mathrm{C}(4)$ & $0.4(4)$ \\
$\mathrm{C}(2)$ & $\mathrm{C}(3)$ & $\mathrm{C}(4)$ & $\mathrm{C}(5)$ & $-1.8(4)$ \\
$\mathrm{C}(3)$ & $\mathrm{C}(4)$ & $\mathrm{C}(5)$ & $\mathrm{C}(6)$ & $1.9(4)$ \\
$\mathrm{C}(4)$ & $\mathrm{C}(5)$ & $\mathrm{C}(6)$ & $\mathrm{C}(1)$ & $-0.6(4)$ \\
$\mathrm{C}(4)$ & $\mathrm{C}(5)$ & $\mathrm{C}(6)$ & $\mathrm{C}(7)$ & $-177.2(2)$ \\
$\mathrm{C}(2)$ & $\mathrm{C}(1)$ & $\mathrm{C}(6)$ & $\mathrm{C}(5)$ & $-0.8(4)$ \\
$\mathrm{S}(1)$ & $\mathrm{C}(1)$ & $\mathrm{C}(6)$ & $\mathrm{C}(5)$ & $179.4(2)$ \\
$\mathrm{C}(2)$ & $\mathrm{C}(1)$ & $\mathrm{C}(6)$ & $\mathrm{C}(7)$ & $176.2(2)$ \\
$\mathrm{S}(1)$ & $\mathrm{C}(1)$ & $\mathrm{C}(6)$ & $\mathrm{C}(7)$ & $-3.6(3)$ \\
$\mathrm{C}(5)$ & $\mathrm{C}(6)$ & $\mathrm{C}(7)$ & $\mathrm{C}(8)$ & $177.1(2)$ \\
$\mathrm{C}(1)$ & $\mathrm{C}(6)$ & $\mathrm{C}(7)$ & $\mathrm{C}(8)$ & $0.4(3)$ \\
$\mathrm{C}(5)$ & $\mathrm{C}(6)$ & $\mathrm{C}(7)$ & $\mathrm{C}(17)$ & $60.3(3)$ \\
$\mathrm{C}(1)$ & $\mathrm{C}(6)$ & $\mathrm{C}(7)$ & $\mathrm{C}(17)$ & $-116.4(2)$ \\
$\mathrm{C}(5)$ & $\mathrm{C}(6)$ & $\mathrm{C}(7)$ & $\mathrm{C}(9)$ & $-63.5(3)$ \\
$\mathrm{C}(1)$ & $\mathrm{C}(6)$ & $\mathrm{C}(7)$ & $\mathrm{C}(9)$ & $119.8(2)$ \\
$\mathrm{C}(6)$ & $\mathrm{C}(7)$ & $\mathrm{C}(8)$ & $\mathrm{O}(1)$ & $-177.0(2)$ \\
$\mathrm{C}(17)$ & $\mathrm{C}(7)$ & $\mathrm{C}(8)$ & $\mathrm{O}(1)$ & $-57.8(3)$ \\
$\mathrm{C}(9)$ & $\mathrm{C}(7)$ & $\mathrm{C}(8)$ & $\mathrm{O}(1)$ & $63.2(3)$ \\
& & & & \\
\hline
\end{tabular}




\begin{tabular}{|c|c|c|c|c|}
\hline $\mathbf{A}$ & B & $\mathbf{C}$ & D & Angle/ ${ }^{\circ}$ \\
\hline $\mathrm{C}(6)$ & $\mathrm{C}(7)$ & $\mathrm{C}(8)$ & $\mathrm{S}(1)$ & $2.8(2)$ \\
\hline $\mathrm{C}(17)$ & $\mathrm{C}(7)$ & $\mathrm{C}(8)$ & $S(1)$ & $122.02(19)$ \\
\hline $\mathrm{C}(9)$ & $\mathrm{C}(7)$ & $\mathrm{C}(8)$ & $S(1)$ & $-116.94(19)$ \\
\hline $\mathrm{C}(1)$ & $\mathrm{S}(1)$ & $\mathrm{C}(8)$ & $\mathrm{O}(1)$ & $175.7(2)$ \\
\hline $\mathrm{C}(1)$ & $\mathrm{S}(1)$ & $\mathrm{C}(8)$ & $\mathrm{C}(7)$ & $-4.15(19)$ \\
\hline$C(6)$ & $\mathrm{C}(7)$ & $\mathrm{C}(9)$ & $\mathrm{C}(11)$ & $170.2(2)$ \\
\hline $\mathrm{C}(8)$ & $\mathrm{C}(7)$ & $\mathrm{C}(9)$ & $\mathrm{C}(11)$ & $-72.4(3)$ \\
\hline $\mathrm{C}(17)$ & $\mathrm{C}(7)$ & $\mathrm{C}(9)$ & $\mathrm{C}(11)$ & $46.4(3)$ \\
\hline$C(6)$ & $\mathrm{C}(7)$ & $\mathrm{C}(9)$ & $C(10)$ & $-62.3(3)$ \\
\hline $\mathrm{C}(8)$ & $\mathrm{C}(7)$ & $\mathrm{C}(9)$ & $C(10)$ & $55.2(3)$ \\
\hline $\mathrm{C}(17)$ & $\mathrm{C}(7)$ & $\mathrm{C}(9)$ & $C(10)$ & $173.9(2)$ \\
\hline $\mathrm{O}(3)$ & $\mathrm{N}(1)$ & $C(10)$ & $\mathrm{C}(9)$ & $-47.0(3)$ \\
\hline $\mathrm{O}(2)$ & $\mathrm{N}(1)$ & $C(10)$ & $\mathrm{C}(9)$ & $135.9(3)$ \\
\hline $\mathrm{C}(11)$ & $\mathrm{C}(9)$ & $\mathrm{C}(10)$ & $\mathrm{N}(1)$ & $-55.8(3)$ \\
\hline $\mathrm{C}(7)$ & $\mathrm{C}(9)$ & $C(10)$ & $\mathrm{N}(1)$ & $175.4(2)$ \\
\hline$C(10)$ & $\mathrm{C}(9)$ & $\mathrm{C}(11)$ & $\mathrm{C}(12)$ & $147.8(2)$ \\
\hline $\mathrm{C}(7)$ & $\mathrm{C}(9)$ & $\mathrm{C}(11)$ & $\mathrm{C}(12)$ & $-86.8(3)$ \\
\hline$C(10)$ & $\mathrm{C}(9)$ & $\mathrm{C}(11)$ & $C(16)$ & $-30.1(3)$ \\
\hline $\mathrm{C}(7)$ & $\mathrm{C}(9)$ & $\mathrm{C}(11)$ & $C(16)$ & $95.3(3)$ \\
\hline$C(16)$ & $\mathrm{C}(11)$ & $\mathrm{C}(12)$ & $\mathrm{C}(13)$ & $-1.4(4)$ \\
\hline $\mathrm{C}(9)$ & $\mathrm{C}(11)$ & $C(12)$ & $\mathrm{C}(13)$ & $-179.4(2)$ \\
\hline $\mathrm{C}(11)$ & $\mathrm{C}(12)$ & $C(13)$ & $C(14)$ & $2.0(4)$ \\
\hline $\mathrm{C}(12)$ & $C(13)$ & $\mathrm{C}(14)$ & $C(15)$ & $-1.4(5)$ \\
\hline$C(13)$ & $C(14)$ & $C(15)$ & $C(16)$ & $0.4(5)$ \\
\hline$C(12)$ & $\mathrm{C}(11)$ & $C(16)$ & $C(15)$ & $0.3(4)$ \\
\hline $\mathrm{C}(9)$ & $\mathrm{C}(11)$ & $C(16)$ & $C(15)$ & 178.2(3) \\
\hline$C(14)$ & $C(15)$ & $C(16)$ & $\mathrm{C}(11)$ & $0.2(4)$ \\
\hline $\mathrm{C}(6)$ & $C(7)$ & $\mathrm{C}(17)$ & $\mathrm{C}(18)$ & $54.4(3)$ \\
\hline $\mathrm{C}(8)$ & $\mathrm{C}(7)$ & $\mathrm{C}(17)$ & $\mathrm{C}(18)$ & $-61.9(3)$ \\
\hline
\end{tabular}




\begin{tabular}{ccccc}
\hline $\mathbf{A}$ & $\mathbf{B}$ & $\mathbf{C}$ & $\mathbf{D}$ & Angle/ \\
\hline $\mathrm{C}(9)$ & $\mathrm{C}(7)$ & $\mathrm{C}(17)$ & $\mathrm{C}(18)$ & $177.8(2)$ \\
$\mathrm{C}(7)$ & $\mathrm{C}(17)$ & $\mathrm{C}(18)$ & $\mathrm{C}(19)$ & $-135.7(3)$ \\
$\mathrm{C}(17)$ & $\mathrm{C}(18)$ & $\mathrm{C}(19)$ & $\mathrm{C}(20)$ & $-178.6(2)$ \\
$\mathrm{C}(18)$ & $\mathrm{C}(19)$ & $\mathrm{C}(20)$ & $\mathrm{C}(25)$ & $170.4(3)$ \\
$\mathrm{C}(18)$ & $\mathrm{C}(19)$ & $\mathrm{C}(20)$ & $\mathrm{C}(21)$ & $-11.5(4)$ \\
$\mathrm{C}(25)$ & $\mathrm{C}(20)$ & $\mathrm{C}(21)$ & $\mathrm{C}(22)$ & $0.7(4)$ \\
$\mathrm{C}(19)$ & $\mathrm{C}(20)$ & $\mathrm{C}(21)$ & $\mathrm{C}(22)$ & $-177.5(2)$ \\
$\mathrm{C}(20)$ & $\mathrm{C}(21)$ & $\mathrm{C}(22)$ & $\mathrm{C}(23)$ & $1.6(4)$ \\
$\mathrm{C}(21)$ & $\mathrm{C}(22)$ & $\mathrm{C}(23)$ & $\mathrm{C}(24)$ & $-2.9(4)$ \\
$\mathrm{C}(21)$ & $\mathrm{C}(22)$ & $\mathrm{C}(23)$ & $\mathrm{N}(2)$ & $175.8(2)$ \\
$\mathrm{O}(5)$ & $\mathrm{N}(2)$ & $\mathrm{C}(23)$ & $\mathrm{C}(22)$ & $-176.7(3)$ \\
$\mathrm{O}(4)$ & $\mathrm{N}(2)$ & $\mathrm{C}(23)$ & $\mathrm{C}(22)$ & $3.5(4)$ \\
$\mathrm{O}(5)$ & $\mathrm{N}(2)$ & $\mathrm{C}(23)$ & $\mathrm{C}(24)$ & $1.9(4)$ \\
$\mathrm{O}(4)$ & $\mathrm{N}(2)$ & $\mathrm{C}(23)$ & $\mathrm{C}(24)$ & $-177.8(3)$ \\
$\mathrm{C}(22)$ & $\mathrm{C}(23)$ & $\mathrm{C}(24)$ & $\mathrm{C}(25)$ & $1.8(4)$ \\
$\mathrm{N}(2)$ & $\mathrm{C}(23)$ & $\mathrm{C}(24)$ & $\mathrm{C}(25)$ & $-176.8(3)$ \\
$\mathrm{C}(23)$ & $\mathrm{C}(24)$ & $\mathrm{C}(25)$ & $\mathrm{C}(20)$ & $0.6(4)$ \\
$\mathrm{C}(21)$ & $\mathrm{C}(20)$ & $\mathrm{C}(25)$ & $\mathrm{C}(24)$ & $-1.8(4)$ \\
$\mathrm{C}(19)$ & $\mathrm{C}(20)$ & $\mathrm{C}(25)$ & $\mathrm{C}(24)$ & $176.4(2)$ \\
\hline
\end{tabular}




\section{Reference.}

[1]. Hooper, T. N.; Butts, C. P.; Green, M.; Haddow, M. F.; McGrady, J. E.; Russell, C. A. Synthesis, Structure and Reactivity of Stable Homoleptic Gold(I) Alkene Cations. Chem. Eur. J. 2009, 15, 12196-12200.

[2]. (a) Ding, M.; Zhou, F.; Liu, Y.-L.; Wang, C.-H.; Zhao, X.-L.; Zhou, J. Cinchona Alkaloid-Based Phosphoramide Catalyzed Highly Enantioselective Michael Addition of Unprotected 3-Substituted Oxindoles to Nitroolefins. Chem. Sci.

2011, 2, 2035-2039. (b) Gao, W.-M.; Yu, J.-S.; Zhao, Y.-L.; Liu, Y.-L.; Zhou, F.; Wu, H.-H.; Zhou, J. Highly

Enantioselective Michael Addition of 3-Arylthio- and 3-Alkylthiooxindoles to Nitroolefins Catalyzed by a Simple Cinchona Alkaloid Derived Phosphoramide. Chem. Commun. 2014, 50, 15179-15182.

[3]. (a) Augusti, R.; Kascheres, C. Reactions of 3-diazo-1,3-dihydro-2H-indol-2-one Derivatives with Enaminones. A Novel Synthesis of 1,2,3-triazoles. J. Org. Chem. 1993, 58, 7079-7083; (b) Shen, J,-H.; Yang, Y.; Hou, X.-L.; Zeng, W.-L.; Yu, A.-M.; Zhao, X.-W.; Meng, X.-T. Darzens Reaction of Thioisatins and Sulfonium Salts: Approach to the Synthesis of Thiochromenone Derivatives with Anticancer Potency. Org. Biomol. Chem. 2018, 16, 3487-3494.

[4]. (a) Romanov-Michailidis, F.; Besnard, C.; Alexakis, A. N-Heterocyclic Carbene-Catalyzed Annulation of a-Cyano-1,4-diketones with Ynals. Org. Lett. 2012, 14, 4906-4909.

[5]. Lang, B.; Zhou, Y.-X.; Lu, P.; Wang, Y.-G. Copper-Catalyzed Synthesis of 3-Allyl-2-Aminoindoles from 3-Diazoindolin-2-Imines and Allyltrimethylsilane. Tetrahedron 2019, 75, 1597-1604.

[6]. (a) Cao, Z.-Y.; Wang, X.; Tan, C.; Zhao, X.-L.; Zhou, J.; Ding, K.-L. Highly Stereoselective Olefin Cyclopropanation of Diazooxindoles Catalyzed by a C2-Symmetric Spiroketal Bisphosphine/Au(I) Complex. J. Am. Chem. Soc. 2013, 135, 8197-8200. (b) Zhao, Y.-L.; Cao, Z.-Y.; Zeng, X.-P.; Shi, J.-M.; Yu, Y.-H.; Zhou, J. Asymmetric Sequential Au(I)/Chiral Tertiary Amine Catalysis: an Enone-Formation/Cyanosilylation Sequence to Optically Active 3-Alkenyloxindoles from Diazooxindoles. Chem. Commun. 2016, 52, 3643-3646. (c) Cao, Z.-Y.; Zhao, Y.-L.; Zhou, J. Sequential Au(I)/Chiral Tertiary Amine Catalysis: A Tandem C-H Functionalization of Anisoles or a Thiophene/Asymmetric Michael Addition Sequence to Quaternary Oxindoles. Chem. Commun. 2016, 52, 2537-2540. (d) Liao, F.-M.; Cao, Z-Y.; Yu, J.-S.; Zhou, J. Highly Stereoselective Gold-Catalyzed Coupling of Diazo Reagents and Fluorinated Enol Silyl Ethers to Tetrasubstituted Alkenes. Angew. Chem. Int. Ed. 2017, 56, 2459-2463.

[7]. Cao, Z.-Y.; Zhang, Y.; Ji, C.-B.; Zhou, J. A $\mathrm{Hg}\left(\mathrm{ClO}_{4}\right)_{2} \cdot 3 \mathrm{H}_{2} \mathrm{O}$ Catalyzed Sakurai-Hosomi Allylation of Isatins and Isatin Ketoimines using Allyltrimethylsilane. Org. Lett. 2011, 13, 6398-6401.

[8]. Trost, B. M.; Zhang, Y.; Zhang, T. Direct N-Carbamoylation of 3-Monosubstituted Oxindoles with Alkyl Imidazole Carboxylates. J. Org. Chem. 2009, 74, 5115-5117.

[9]. Zhou, B.; Luo, Z.; Li, Y. Assembly of Spirooxindole Derivatives Containing Four Consecutive Stereocenters by Using Cascade Reactions Catalyzed by an N - Heterocyclic Carbene. Chem. Eur. J. 2013, 19, 4428-4431.

[10]. Pan, Y.-L.; Zheng, H.-L.; Wang, J.; Yang, C.; Li, X.; Cheng, J.-P. Enantioselective Allylation of Oxocarbenium Ions Catalyzed by $\mathrm{Bi}(\mathrm{OAc})_{3} /$ Chiral Phosphoric Acid. ACS Catal. 2020, 10, 8069-8076. 